Noname manuscript No.

(will be inserted by the editor)

Sungmin Hwang • Benjamin

Schmiegelt · Luca Ferretti · Joachim

Krug

\title{
Universality classes of interaction structures for NK fitness landscapes
}

Received: date / Accepted: date

\begin{abstract}
Kauffman's NK-model is a paradigmatic example of a class of stochastic models of genotypic fitness landscapes that aim to capture generic features of epistatic interactions in multilocus systems. Genotypes are represented as sequences of $L$ binary loci. The fitness assigned to a genotype is a sum of contributions, each of which is a random function defined on a subset of $k \leq L$ loci. These subsets or neighborhoods determine the genetic interactions of the model. Whereas earlier work on the NK model suggested that most of its properties are robust with regard to the choice of neighborhoods, recent work has revealed an important and sometimes counter-intuitive influence of the interaction structure on the properties of NK fitness landscapes. Here we review these developments and present new results concerning the number of local fitness maxima and the statistics of selectively accessible (that is, fitness-monotonic) mutational pathways. In particular, we develop a unified framework for computing the exponential growth rate of the expected number of local fitness maxima as a function of $L$, and identify two different universality classes of interaction structures that display different asymptotics of this quantity for large $k$. Moreover, we show that the probability that the fitness landscape can be traversed along an accessible path decreases exponentially in $L$ for a large class of interaction structures that we characterize as locally bounded. Finally, we discuss the impact of the
\end{abstract}

S. Hwang · B. Schmiegelt · J. Krug

Institute for Theoretical Physics, University of Cologne

Tel.: +492212818

Fax: +492215159

E-mail: hwang@thp.uni-koeln.de, schmiegb@thp.uni-koeln.de, krug@thp.unikoeln.de

Present address of S. Hwang: LPTMS, Université Paris-Sud 11, Orsay, France

L. Ferretti

Integrative Biology group, The Pirbright Institute, United Kingdom

E-mail: luca.ferretti@pirbright.ac.uk 
NK interaction structures on the dynamics of evolution using adaptive walk models.

Keywords Evolution, fitness landscapes, epistasis, adaptive walks

\section{Introduction}

\subsection{Probabilistic models of fitness landscapes}

Biological evolution can be conceptualized as a search process in the space of gene sequences guided by the fitness landscape, a mapping that assigns a measure of reproductive value to each genotype [40,83,89. The relationship between genotype and fitness is exceedingly complex, as it is mediated in a highly nonlinear way by the multidimensional organismic phenotype that interacts with the environment and thereby determines reproductive success. A common strategy to deal with this complexity is to shortcut the intermediate phenotypic level by assigning fitness directly to genotypes. This leads to probabilistic models that define fitness landscapes in terms of ensembles of random functions on a suitably chosen discrete space 81 . The idea that unmanageable complexity can be replaced by randomness is familiar from the statistical physics of disordered systems, and there are strong links between the two fields 82 .

The prime example of a genotype space is the Hamming graph $\mathbb{H}_{A}^{L}$, the set of all sequences of length $L$ with symbols taken from an alphabet of size $A$ and equipped with the Hamming metric which counts the number of symbols in which two sequences differ. The alphabet size is $A=4$ for nucleotide sequences and $A=20$ for proteins. In the context of classical genetics $A$ denotes the number of alleles that can be present at a certain genetic locus. Many studies including the present one restrict their scope to binary sequences with $A=2$, where the corresponding binary sequence space $\mathbb{H}_{2}^{L}$ is an $L$-dimensional hypercube.

The probabilistic approach was pioneered by Kauffman and Levin 35, who considered the conceptually simplest case where fitness values of different genotypes are drawn independently from a common probability distribution. With reference to an earlier publication by Kingman where a similar scheme was introduced in a setting with an infinite number of alleles [39, the uncorrelated model is known as the House-of-Cards landscape (HoC). In the words of Kingman, the rationale behind this term is the idea that any mutation completely destroys "the biochemical 'house of cards' built up by evolution". The assumption that a single mutation leads to a fitness value for the offspring that is uncorrelated with the parent is clearly unrealistic, and indeed recent empirical studies have shown that the $\mathrm{HoC}$ model overestimates the ruggedness of real fitness landscapes $28,50,84,89,94$. In subsequent work, Kauffman and collaborators therefore devised a class of fitness landscape models known as NK models in which the correlation between fitness values can be tuned 36, 37. The construction of these models was clearly influenced by the concurrent (though somewhat earlier) developments in the theory of 
disordered systems [82, as evidenced by the frequent references to spin glasses in the original paper [37].

\subsection{NK models and ruggedness}

In NK fitness landscapes, the fitness is written as a sum of contributions, each of which depends in a HoC-like fashion on a subset of loci. As a consequence, a mutation at a particular locus changes only the contributions of those subsets that contain this locus, whereas all other contributions remain unchanged. In this way, the level of fitness correlations can be controlled through the size and composition of the interacting subsets. In the original formulation of the model, the number of subsets is taken to be equal to the number of loci, and each subset is associated to a specific locus which it contains together with $K$ others. In later work some of these constraints have been relaxed 2 , 50, and below in Sec. 2 we provide a formal definition of the model that allows to incorporate various generalizations in a unified way. NK fitness landscapes constructed according to the original version of the model will be referred to as classical. Even within the set of classical NK landscapes there are obviously many distinct, deterministic or stochastic schemes by which loci can be assigned to interacting subsets. This assignement is the key structural degree of freedom of the NK model, and can be viewed as a crude representation of genetic architecture. For convenience, our nomenclature differs in two respects from that of the original definitions of Kauffman and coworkers: First, we denote the number of loci by $L$ rather than $N$; second, the size of interacting subsets is denoted by $k=K+1$ throughout this article.

Since its introduction three decades ago the NK-model has been widely applied in investigations of fundamental questions of evolutionary theory [56, 61,96] as well as for the analysis of empirical fitness landscapes [75]. But also beyond the original context of evolutionary biology, the model provides a remarkably versatile framework for exploring how structural constraints give rise to diversity and complexity in the solution spaces of various optimization problems. Correspondingly, NK fitness landscapes appear in fields ranging from evolutionary computation to management science and economics [6, 42, 46, 74, 87, 99 .

Much of the extensive, if somewhat scattered literature has investigated features of NK fitness landscapes that are relevant to the efficiency of mutational searches, particularly the statistics of fitness maxima [6, 14, 15, 43, 92. At least under conditions of low mutation supply where populations explore the landscape through single mutational steps, local fitness maxima present obstacles to the search process, and their role in slowing down evolutionary progress has been a concern in evolutionary theory ever since the fitness landscape concept was first introduced in the 1930's [27,100. The existence of multiple fitness peaks is therefore the criterion that is most commonly used to specify what it means for a fitness landscape to be rugged 11,70, 89.97. In the related context of spin glasses, the fitness peaks correspond to metastable states 27. that govern the low-temperature behavior of these systems [12,57]. 
Recent theoretical and empirical studies have identified alternative measures of fitness landscape ruggedness that focus on the mutational pathways along which local or global fitness peaks can be reached $9,68,84,89,95$. Under conditions of low mutation supply and large fitness differences, mutational pathways are accessible to the evolving population only if fitness increases monotonically along the path, a condition that often strongly reduces the combinatorial abundance of possible evolutionary trajectories implied by the high connectivity of genotype space 4, 19,20,28, 29,53, 93, The basic evolutionary dynamics in this regime is captured by adaptive walk models, in which a genetically homogeneous (monomorphic) population moves towards higher fitness along the network of accessible pathways in single mutational steps [25, 35, 45, 54,58. Adaptive walks terminate at local fitness maxima, and the number of steps required to reach a maximum from a random starting point is a convenient measure of landscape ruggedness. At least in order of magnitude, the length of adaptive walks is expected to be comparable to the typical distance between maxima and also to the correlation length of the fitness correlation function $[54,73,80,81,92$.

\subsection{Aims and scope}

In this article we review our current understanding of how the ruggedness of NK fitness landscapes, as quantified by the number of fitness peaks, the number of accessible paths and the length of adaptive walks, depends on the parameters of the landscape. These parameters comprise the following elements:

- the number of loci $L$ and the size $k$ of interacting groups of loci;

- the scheme according to which loci are assigned to groups, henceforth referred to as the NK structure of the model; and

- the probability distribution from which the fitness values assigned to the configurations of the interacting groups are drawn.

Early numerical work on NK landscapes suggested that the number of fitness peaks and the length of adaptive walks is determined primarily by the parameters $k$ and $L$, with little or no dependence on the NK structure. The two specific structures considered were the adjacent neighborhood model (AN), where the loci belonging to the same interacting subset are adjacent along the sequence, and the random neighborhood model $(\mathrm{RN})$ where the members of each group are chosen at random among all loci. Based on simulations of these two models Kauffman writes that "whether the $K$ epistatic inputs to a gene are its neighbors or random among the $N$ has almost no bearing on the lengths of walks to optima" [36. Weinberger concluded from an approximate analytic investigation that "the topography of $N-k$ landscapes seems to be independent of how the neighborhoods are chosen" 92, and similar statements can still be found in the current literature 85. Some support for this hypothesis derives from the fact that the fitness correlation function of classical NK-landscapes has a universal form that is completely specified by $k$ and $L[7,8,50$. 
On the other hand, recent numerical simulations of accessible pathways and adaptive walks revealed significant differences between different NK structures 54,78, and a survey of earlier work suggested that similar (if less pronounced) differences exist also with regard to the statistics of fitness peaks. The handful of available exact results for the asymptotic growth rate of the number of maxima with $L$ display a distinct dependence on the fitness distribution which gives way to universal behavior only when $k$ is large [14, 15, 43, 54. Since these rigorous analyses were restricted to the AN model, no conclusions could be drawn with regard to the dependence on the interaction structure. The latter was addressed numerically by Buzas and Dinitz, who found a correlation between the number of fitness peaks and the rank of the structure [6, 54. The rank is a measure of the connectivity of the genetic architecture that will be formally defined below in Sec. 2.6.

In the next section we introduce the mathematical framework needed to define the quantities and models of interest. We then embark on a detailed investigation of the mean number of local fitness maxima in NK landscapes, focusing specifically on the exponential growth rate $\lambda_{k}$ of this quantity for large $L$. Starting from two exactly solvable cases, the block model (BN) where the interacting subsets are disjoint [60,66,78, and a novel mean field model (MF) where all possible subsets contribute to the fitness landscape with equal weights, we identify two classes of NK structures characterized by distinct asymptotic behaviors of $\lambda_{k}$ for large $k$. We systematize and expand the range of exact expressions that have been reported for $\lambda_{k}$ for the AN model, which is known from previous work to share the asymptotic behavior of the BN model. We then demonstrate that the $\mathrm{RN}$ model falls into the class of the $\mathrm{MF}$ model, thus establishing that $\mathrm{AN}$ and $\mathrm{RN}$ structures are not equivalent even for large $k$.

In Sections 4 and 5 we review the state of the art regarding accessible pathways and adaptive walks in NK fitness landscapes, and sketch a proof of the asymptotic absence of globally accessible pathways for a large class of NK structures. Finally, Section 6 summarizes the paper and provides an outlook on open problems. Some derivations and proofs and a description of the numerical algorithm used to count the number of fitness maxima are relegated to Appendices.

\section{Mathematical background and definitions}

\subsection{Genotype space}

We assume that the genome of an individual consists of a fixed number $L$ of independently mutable loci labeled by an index set $\mathcal{L}=\left\{l_{1}, \ldots, l_{L}\right\}$, called the locus set. Generally each locus could be found in many different states, or alleles. For simplicity it is usually assumed that each locus can be found in the same number $A$ of states labeled $\left\{a_{1}, \ldots, a_{A}\right\}$. Here however, as mentioned in the introduction, we focus on the case $A=2$, choosing $a_{1}=1$ and $a_{2}=-1$ as the only possible alleles at each locus. These may e.g. be interpreted as the wild type and a mutated type. A genotype corresponds to an assignment of alleles to each locus, or equivalently (assuming an ordering 
of $\mathcal{L}$ ) a sequence of alleles, i.e. for $A=2$ a sequence of $L$ binary values $\sigma=\left\{\sigma_{l_{1}}, \ldots \sigma_{l_{L}}\right\} \in\{ \pm 1\}^{L}$. The space of all genotypes will be denoted $\mathbb{H}_{\mathcal{L}}$. Taking the genotype space as a vertex set for a simple undirected graph and drawing edges between any two genotypes differing at exactly one locus, we arrive at the Hamming graph $\mathbb{H}_{2}^{L}$, the $L$-fold Cartesian graph product of the complete graph on two vertices. For higher values of $A$, the resulting graph would have been the Hamming graph $\mathbb{H}_{A}^{L}$, the $L$-fold Cartesian graph product of the complete graph on $A$ vertices. This mutation graph defines all possible changes in genotypes due to single point mutations. While it is possible for an offspring to accumulate multiple point mutations relative to its parent, if the mutation rate is small in comparison to the inverse of the product of $L$ and the population size, then double mutants are unlikely to appear and an asexual population may only explore the genotype space by single steps along the Hamming graph. In this regime, the Hamming graph is indeed the graph of all possible mutational transitions.

The graph metric of the Hamming graph is the Hamming metric

$$
d_{h}(\sigma, \theta)=\sum_{i=1}^{L}\left(1-\delta_{\sigma_{i} \theta_{i}}\right)
$$

measuring the number of loci at which two genotypes differ and thereby the minimal number of mutational steps needed to be taken to reach one from the other. We define the operators $\Delta_{l}: \mathbb{H}_{\mathcal{L}} \rightarrow \mathbb{H}_{\mathcal{L}}$ for all $l \in \mathcal{L}$ such that

$$
\left(\Delta_{l} \sigma\right)_{m}=\left(1-2 \delta_{l m}\right) \sigma_{m} .
$$

This (single-locus) mutation operator switches the allele at the $l$-th locus of a genotype, corresponding to one edge attached to $\sigma$ in the Hamming graph. These operators are then extended to (multi-locus) mutation operators $\Delta_{\mathcal{M}}$ for all $\mathcal{M} \subseteq \mathcal{L}$, such that $\Delta_{\mathcal{M}}=\prod_{l \in \mathcal{M}} \Delta_{l}$. Because loci are mutationally independent the order of operations in the product does not matter and all mutation operators commute. Furthermore mutation operators are selfinverse and form a group that leaves the metric invariant,

$$
d_{h}(\sigma, \theta)=d_{h}\left(\Delta_{\mathcal{M}} \sigma, \Delta_{\mathcal{M}} \theta\right),
$$

and

$$
d_{h}\left(\sigma, \Delta_{\mathcal{M}} \sigma\right)=|\mathcal{M}|
$$

The maximal distance between two genotypes on the Hamming graph is $L$. For each genotype $\sigma$, there is exactly one genotype at this distance, the antipode $\Delta_{\mathcal{L}} \sigma$. If two genotypes share an edge in the mutation graph, or equivalently lie at Hamming distance 1 , then we say they are adjacent. A sequence of adjacent genotypes $\left(\sigma^{(0)}, \ldots, \sigma^{(n)}\right)$ is called a (mutational) path(-way). Here $\sigma^{(0)}$ is the initial genotype and $\sigma^{(n)}$ the final genotype, and $n$ is the path length. Each path may also be expressed as an initial genotype together with a sequence of $n$ loci $\left(m_{1}, \ldots, m_{n}\right) \in \mathcal{L}^{n}$, so that $\sigma^{(i)}=\Delta_{m_{i}} \sigma^{(i-1)}$. Here we require, if not mentioned otherwise, paths to be simple. This means paths may not visit any genotype more than once. We apply this constraint because accessible pathways, which will be discussed in more detail in Sec. 4 , are strictly fitness increasing and thus can never loop back to a previous genotype. 


\subsection{Fitness landscapes}

A fitness landscape is a mapping $F: \mathbb{H}_{\mathcal{L}} \rightarrow \mathbb{R}$ assigning each genotype a real-valued fitness. Starting from an initial genotype $\sigma$, a mutation $\Delta_{\mathcal{M}}$ induces a fitness change which we will write in the shorthand notation

$$
\Delta_{\mathcal{M}} F(\sigma)=F\left(\Delta_{\mathcal{M}} \sigma\right)-F(\sigma) .
$$

The operator $\Delta_{\mathcal{M}}$ may be understood here as a difference operator mapping a fitness landscape to a function which assigns to each genotype the selection coefficient associated with application of the set of mutations $\mathcal{M}$.

Asexual populations may be viewed as distributions on the genotype space. Due to selection these distributions typically tend to move towards higher fitness and stagnate at local fitness maxima of the fitness landscape. Mutation and genetic drift introduce noise resulting in distributions of finite width (in terms of genotype distance). If selection is significantly outweighing the mutational input, then this width will be very small and populations are effectively localized at exactly one majority genotype. Over time mutations will occur, which, due to strong selection, will fixate to become the new majority genotype if and only if they increase fitness. The resulting dynamics is that of an adaptive walk, a time- and space-discrete Markov process over the genotype space, where the population moves stepwise in the direction of strictly increasing fitness (see Sec. 5).

To describe not the actual probabilities, but rather only the possibility of such a walk taking certain mutational paths, it is useful to introduce the reduced notion of a fitness graph. The fitness graph of a fitness landscape is the orientation of the mutation graph $\mathbb{H}_{\mathcal{L}}$, such that arrows point towards higher fitness [11,19, 90]. For convenience we will assume that no two genotypes have exactly the same fitness, i.e. $\Delta_{\mathcal{M}} F(\sigma) \neq 0$ for all $\sigma$ and $\Delta_{\mathcal{M}}$. Then the fitness graph is well-defined and acyclic (see Fig. 8 for some simple examples).

The fitness graph contains only information about signs of local mutation effects and as such may not convey enough information about the original fitness landscape. For example local maxima can be identified from the fitness graph, but the global one cannot be determined. As an intermediate reduction one may consider only ranks of fitness values: The ranked fitness landscape $\mathcal{R}[F]$ of a fitness landscape $F$ is again a fitness landscape, such that $\mathcal{R}[F](\sigma)$ is the rank of $F(\sigma)$ if all $2^{L}$ fitness values are ordered in ascending order $[10$. The ranked fitness landscape's fitness graph is the same as that of the original landscape.

Despite recent progress in the large-scale analysis of empirical fitness landscapes [3,41,71, most available data sets are restricted to small numbers of loci $28,84,89,94$, and measuring fitness landscapes on a genome-wide level remains an insurmountable challenge. We also cannot hope to describe specific landscapes exactly from their underlying biological and chemical structure. Thus the approach taken is to consider probabilistic models of fitness landscapes, based on theoretical or empirical principles, to describe typical properties of such landscapes. Let $\mathbb{F}_{\mathcal{L}}=\mathbb{R}^{\mathbb{H}_{\mathcal{L}}}$ be the space of all fitness land- 
scapes over the locus set $\mathcal{L}$. Then a fitness landscape model is a probability measure over $\mathbb{F}_{\mathcal{L}}$.

Several such models have been studied. From a mathematical viewpoint, the simplest non-trivial model is probably the House-of-Cards (HoC) model 35. In this model all fitness values $\{F(\sigma)\}_{\sigma \in \mathbb{H}_{\mathcal{L}}}$ are chosen i.i.d. from some continuous real-valued base fitness distribution $p_{f}$. Continuity guarantees that almost surely no two fitness values are equal. The HoC model's ranked fitness landscape is independent of the actual choice of $p_{f}$, reducing the calculation of ranked properties, such as the number of local maxima, to combinatorial problems.

The HoC model, however, does not allow for correlations between mutational effects on the same locus and thus lacks a structure on loci. One possible (though most extreme way) of associating fitness benefits with certain alleles at specific loci is to assign fitness values $f_{l}\left(\sigma_{l}\right)$ to each allele of each locus and define the total fitness as

$$
F(\sigma)=\sum_{l \in \mathcal{L}} f_{l}\left(\sigma_{l}\right)
$$

If the values of $f_{l}$ are chosen i.i.d. from a continuous probability distribution, then $f_{l}$ is effectively a HoC landscape over one locus. This linear model is the opposite extreme of the HoC landscape. Given the fitness difference between two alleles on one background, the fitness effect on every other background is identical, i.e. $\Delta_{l} F(\sigma)=\Delta_{l} f_{l}\left(\sigma_{l}\right)$ depends only on $\sigma_{l}$.

A canonical way of quantifying the degree of correlation in a fitness landscape model is through the distance correlation function $\rho(d)$ defined as 80,81

$$
\rho(d)=\frac{\mathbb{E}_{d_{h}(\sigma, \theta)=d}[F(\sigma) F(\theta)]-\mathbb{E}_{\sigma}[F(\sigma)]^{2}}{\mathbb{E}_{\sigma}\left[F(\sigma)^{2}\right]-\mathbb{E}_{\sigma}[F(\sigma)]^{2}},
$$

where the one-point expectations are taken over all $\sigma \in \mathbb{H}_{\mathcal{L}}$ and the two-point expectations over all combinations of $\sigma \in \mathbb{H}_{\mathcal{L}}$ and $\theta \in \mathbb{H}_{\mathcal{L}}$ such that their Hamming distance is exactly $d$. For the HoC model $\rho(d)=\delta_{d, 0}$, whereas for the linear model $\rho(d)=1-d / L$.

\subsection{Epistasis}

The linear model is non-epistatic, meaning that each mutation has a fixed effect on overall fitness, independent of the states of other loci. In contrast epistasis refers to the dependence of mutational effects on the state of other loci [16, 67, 88. Formally we say that two loci $l$ and $m$ are epistatic (for a genotype $\sigma$ ), if

$$
\Delta_{l} F(\sigma) \neq \Delta_{l} F\left(\Delta_{m} \sigma\right) .
$$

It is useful to further differentiate magnitude and sign epistasis 95. Sign epistasis is present if the equation above also hold after application of the sign function on both sides, i.e. if

$$
\operatorname{sgn} \Delta_{l} F(\sigma) \neq \operatorname{sgn} \Delta_{l} F\left(\Delta_{m} \sigma\right) .
$$


In this case mutations on $m$ can affect whether mutations on $l$ are beneficial or not. If sign epistasis is not present, then there is only magnitude epistasis, in which $m$ can affect the quantitative benefit of a mutation on $l$, but cannot change it from beneficial to deleterious. In this case it is easy to show that the fitness landscape has a unique maximum [95. Note that $l$ is epistatic with $m$ if $m$ is epistatic with $l$, but the same is not true for sign epistasis. If however $l$ is sign epistatically dependent on $m$, as well as the other way around, then one speaks of reciprocal sign epistasis 68, 70, see Sec. 4 for further discussion.

An alternative description of epistasis as function of distance on the hypercube is provided by the $\gamma$ statistic introduced in 16. For a given focal mutation $l$ and a set of mutations $\mathcal{M}$, it is defined as the correlation between fitness effects of parallel transported arrows in the fitness graph,

$$
\gamma_{l, \mathcal{M}}=\frac{\operatorname{Cov}\left[\Delta_{l} F(\sigma), \Delta_{l} F\left(\Delta_{\mathcal{M}} \sigma\right)\right]}{\mathbb{E}\left[\left(\Delta_{l} F(\sigma)\right)^{2}\right]}
$$

where the mean and covariance are taken over all (or a subset of) genotypes $\sigma$. For the case when $\mathcal{M}$ consists of a single locus $\mathcal{M}=\{m\}, \gamma_{l, m}$ quantifies the average strength of epistasis on mutation $\Delta_{l}$ due to prior application of mutation $\Delta_{m}$. Different values of $\gamma_{l, m}$ indicate the prevalence of no, magnitude-only, sign- or reciprocal epistasis for $\gamma_{l, m}=1,1>\gamma_{l, m}>0$, $1>\gamma_{l, m}>-\frac{1}{3}$ and $\gamma_{l, m}<0$, respectively.

\subsection{Fourier-Walsh decomposition}

Being functions over a finite commutative group, fitness landscapes admit a Fourier decomposition of the form $[73,81,91$

$$
F(\sigma)=\sum_{g \in \wp(\mathcal{L})} \hat{F}(g) \prod_{l \in g} \sigma_{l},
$$

where $\wp$ denotes the power set and the $\hat{F}(g)$ are Fourier coefficients. As there are $2^{L}$ subsets of $\mathcal{L}$, the mapping between the fitness values $F(\sigma)$ and the Fourier coefficients $\hat{F}(g)$ is one-to-one and invertible. The decomposition Eq. (11) is an expansion in eigenfunctions of the graph Laplacian of the hypercube, which is also known as a Walsh transform in computer science 94.

The linear fitness landscape Eq. (6) is a special case of Eq. (11) where the $\hat{F}(g)$ are nonzero only when $g$ is the empty set or a single locus. Correspondingly, terms containing products of $p \geq 2$ locus contributions encode epistatic interactions of order $p$. Specifically, $\hat{F}(g)$ is proportional to the $|g|$-way epistasis among the loci in the subset $g$ averaged over all genetic backgrounds 69. The Fourier spectrum of a fitness landscape is obtained by summing the squares of the Fourier coefficients for each order $p$, which provides a measure for the strength of epistasis of different orders [50,94. Note, however, that the presence or absence of sign epistasis depends on the specific 
values of the coefficients $\hat{F}(g)$ and cannot be read off from the Fourier spectrum. The Fourier spectrum is related to the distance correlation function Eq. (7) through a one-dimensional linear mapping involving discrete orthogonal polynomials [81.

\subsection{Local maxima}

A local fitness maximum is a genotype $\sigma$, such that all single-locus mutations have lower fitness than $\sigma$, i.e. such that $\Delta_{l} F(\sigma)<0$ for all $l \in \mathcal{L}$. Thus a local maximum is a sink in the fitness graph. Different concepts of local maxima may be used, e.g. one could require $\Delta_{l} F(\sigma)<-\epsilon$ for some $\epsilon>0$ as to limit the definition to more selectively robust maxima. Since one can have double mutants for sufficiently large mutation rate, it may also be of interest to consider maxima which are robust up to higher distance, i.e. $\sigma$ with $\Delta_{\mathcal{M}} F(\sigma)<0$ for all $\mathcal{M} \subset \mathcal{L}$ such that $|\mathcal{M}| \leq D$, where $D$ is the number of simultaneous mutations considered. Here we will only consider the simple first definition.

We will denote the expected number of local maxima as $\#_{\max }$, possibly with an index describing the model. There are $2^{L}$ genotypes and thus the fraction of genotypes expected to be local maxima can be written $\pi_{\max }=$ $2^{-L} \#_{\max }$. Provided the fitness landscape model of interest is homogeneous, in the sense that all genotypes are statistically equivalent, $\pi_{\max }$ is also the probability that a randomly chosen genotype is a local maximum. We will use this in Sec. 3 to study the expected number of local maxima. Two examples of fitness landscape models that are not homogeneous can be found in 30 . 51.

\subsection{NK model}

Both the HoC and the linear model are extreme cases. Realistically we expect some intermediate structure with some ruggedness but still correlated mutation effects. The idea of Kauffman's NK model 36,37 is to introduce a parameter $k$ to the system, which is able to interpolate between the HoC and the linear model. The model is constructed starting from the linear model Eq. (6). However each fitness contribution $f_{l}$ is now not only dependent on $\sigma_{l}$, but also on the states of an additional set of $k-1$ other loci. The concrete choice of these additional loci may vary and will be discussed later. The fitness values of the fitness contributions $f_{l}$, now functions of $k$ alleles, are then assumed to be randomly distributed in accordance with the HoC model. In this way $f_{l}$ can still be interpreted as the fitness contribution of locus $l$, but now being dependent on a few other locus states. At $k=1$, there are no additional locus dependencies and the linear model is retrieved. For $k=L$, each $f_{l}$ must necessarily be a HoC landscape over all of $\mathcal{L}$ and thus $F(\sigma)$ is itself a HoC landscape. Intermediate values of $k$ are able to interpolate between these cases or between different amounts of ruggedness.

We will however define a generalization of the NK model first. The (generalized) NK model over a locus set $\mathcal{L}$ is parametrized by a multiset $\mathcal{B}$ 

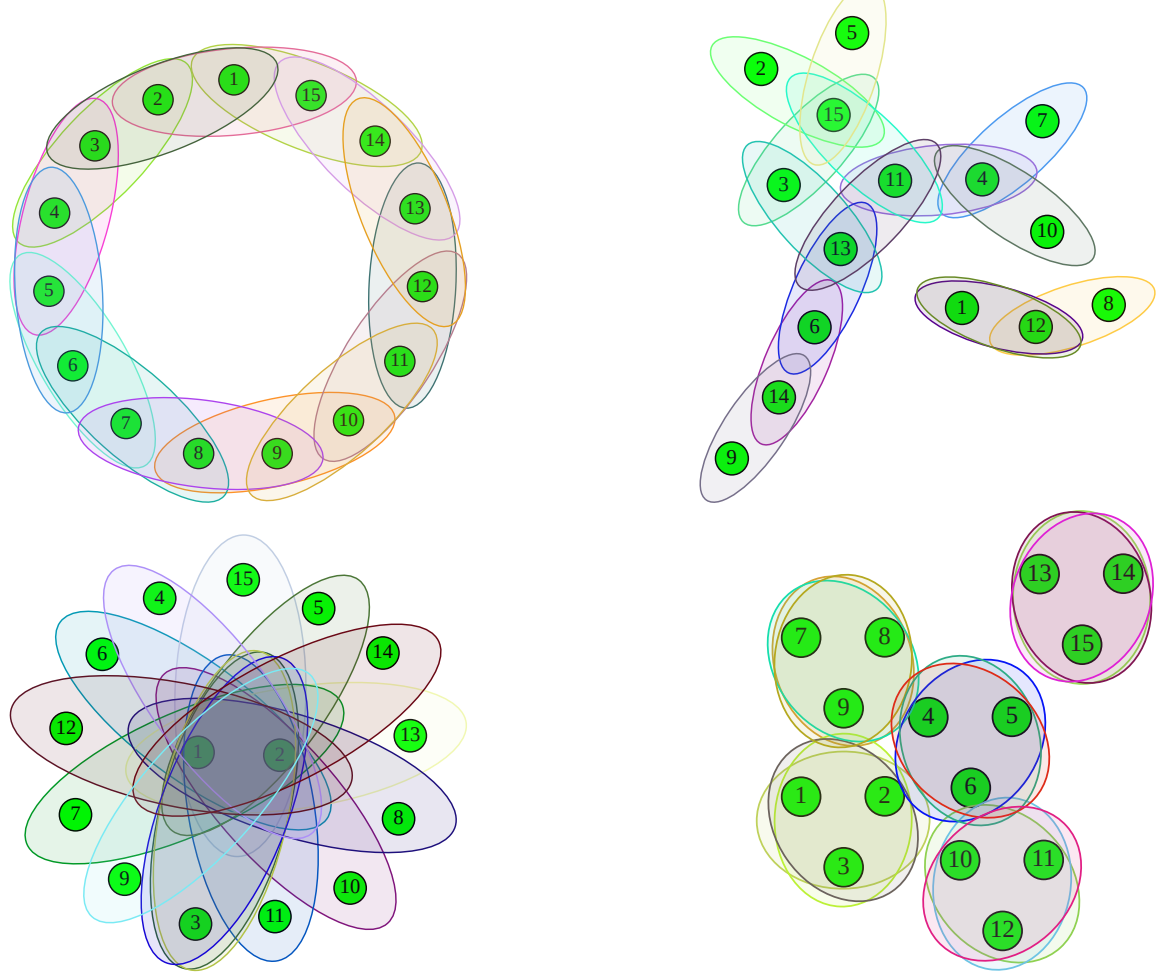

Fig. 1 Examples of NK structure hypergraphs. Each ellipse represents one NK edge and nodes represent different loci. Top left: AN structure with $k=3$ and $L=15$. Top right: uRN structure with $k=2$ and $L=15$. Bottom left: SN structure with $k=3$ and $L=15$. Bottom right: BN structure with $k=3$ and $L=15$. We choose $k=2$ for the $\mathrm{uRN}$ model for the sake of readability. For higher $k$ uRN structures are usually not planar anymore.

containing subsets of $\mathcal{L}$. This multiset can be interpreted as the edge set of a (multi-)hypergraph over the set of loci $\mathcal{L}$ (Fig. 1). We call this hypergraph the NK structure (hypergraph) and its edge sets (the elements of $\mathcal{B}$ ) NK edges, NK blocks or NK neighborhoods. By $|\mathcal{B}|$ we denote the total number of elements (multiplicities included) of $\mathcal{B}$ and we index the NK edges (in some fixed manner) by natural numbers $\{1, \ldots,|\mathcal{B}|\}$, i.e. $B_{1} \ldots B_{|\mathcal{B}|}$. Then we assign to edge $B_{i}$ a HoC landscape $f_{i}$ over $\mathbb{H}_{B_{i}}$, i.e. a completely random landscape over a subset of loci. Finally the total fitness is defined as

$$
F(\sigma)=\sum_{i=1}^{|\mathcal{B}|} f_{i}\left(\downarrow_{B_{i}} \sigma\right) .
$$

Here $\downarrow_{B_{i}} \sigma$ is the projection of $\sigma$ onto the subset of loci $B_{i}$, i.e. $\downarrow_{\mathcal{M}}: \mathbb{H}_{\mathcal{L}} \rightarrow \mathbb{H}_{\mathcal{M}}$ such that $\left(\downarrow_{\mathcal{M}} \sigma\right)_{m}=\sigma_{m}$ for all $m \in \mathcal{M}$. The projection of a genotype onto $\mathcal{M}$ retains all alleles at loci in $\mathcal{M}$, but discards all other loci in $\mathcal{L} \backslash \mathcal{M}$. The orthogonal projection $\downarrow_{\mathcal{L} \backslash \mathcal{M}}$ yields those alleles that have been discarded 
by $\downarrow_{\mathcal{M}}$, and $\downarrow_{\mathcal{L} \backslash \mathcal{M}} \sigma$ is called the background genotype of $\sigma$ relative to the projection onto $\mathcal{M}$. The union (in the sense of relations) of the two orthogonal projections returns the original genotype. Consider for example a locus set $\mathcal{L}=\left\{l_{1}, l_{2}, l_{3}, l_{4}\right\}$ and an NK edge $\left\{l_{2}, l_{3}\right\} \subseteq \mathcal{L}$. The projection of genotype $(-1,-1,1,1)$ onto the edge is then $(-1,1)$ (assuming ordering as above).

Partially in order to avoid certain inconvenient edge cases we make the following restrictions on the NK structure:

1. For every $l \in \mathcal{L}$ there exists a $B_{i}$ with $l \in B_{i}$. This assures that there are no neutral mutations and that no two fitness values are equal, almost surely.

2. $\frac{1}{|\mathcal{B}|} \sum_{i=1}^{|\mathcal{B}|}\left|B_{i}\right|=k$, where $k$ is a constant generalizing the parameter $k=$ $K+1$ in the original NK model.

Together they imply that $|\mathcal{B}| k \geq L$.

There are obviously many possible choices of the interactions, however some specific further conditions are of interest. First note that the partial landscape $f_{i}$ only contributes to a mutation effect $\Delta_{l} F(\sigma)$ if $l \in B_{i}$. The effects on those partial landscapes are all identical and independent and thus we have

$$
\mathbb{E}\left[\Delta_{l} F(\sigma)\right]=0
$$

and

$$
\operatorname{var}\left[\Delta_{l} F(\sigma)\right]=2 \sigma_{f} \cdot\left|\left\{B_{i} \in \mathcal{B} \mid l \in B_{i}\right\}\right|
$$

where $\sigma_{f}$ is the variance of the base fitness distribution and the second term counts the number of NK edges containing $l$. The distribution of this variance over loci is important to the behavior of the model. In the most extreme case the variance of few a loci may be on the order of $L$, while other loci are contained only in one NK edge each. Then the high-variance loci will mostly determine the fitness of a genotype, while the other loci only introduce slight variations. Such a high-variance locus would be largely independent of the state of other loci. An example for this kind of structure will be introduced below in Sec. 2.7. In contrast, if each locus appears in an equal number of NK edges, all loci have equal-variance effects and none is special. We call such a structure regular. Due to the definition of $k$, the common number of NK edges containing a specific locus is then $k|\mathcal{B}| / L$.

We say an NK structure is uniform if $\left|B_{i}\right|=k$ for all $B_{i}$. This is equivalent to the hypergraph being $k$-uniform. For uniform structures the Fourier decomposition Eq. (11) contains products of locus variables up to order $k$ only.

We say a uniform NK structure is classical if $|\mathcal{B}|=L$ and $l \in B_{l}$ for all $l \in \mathcal{L}$. This definition encompasses the class of NK models considered in the original articles by Kauffman et al. 36, 37. This subclass has nice properties which allow for a simpler graphical representation of the NK structure: the simplified NK structure (graph) is the directed simple graph over $\mathcal{L}$ with an arrow from $l$ to $m$ if $l \in B_{m}$ (Fig. 2). Campos et. al. 7,8 show that the distance correlation function is independent of the concrete structure choice 

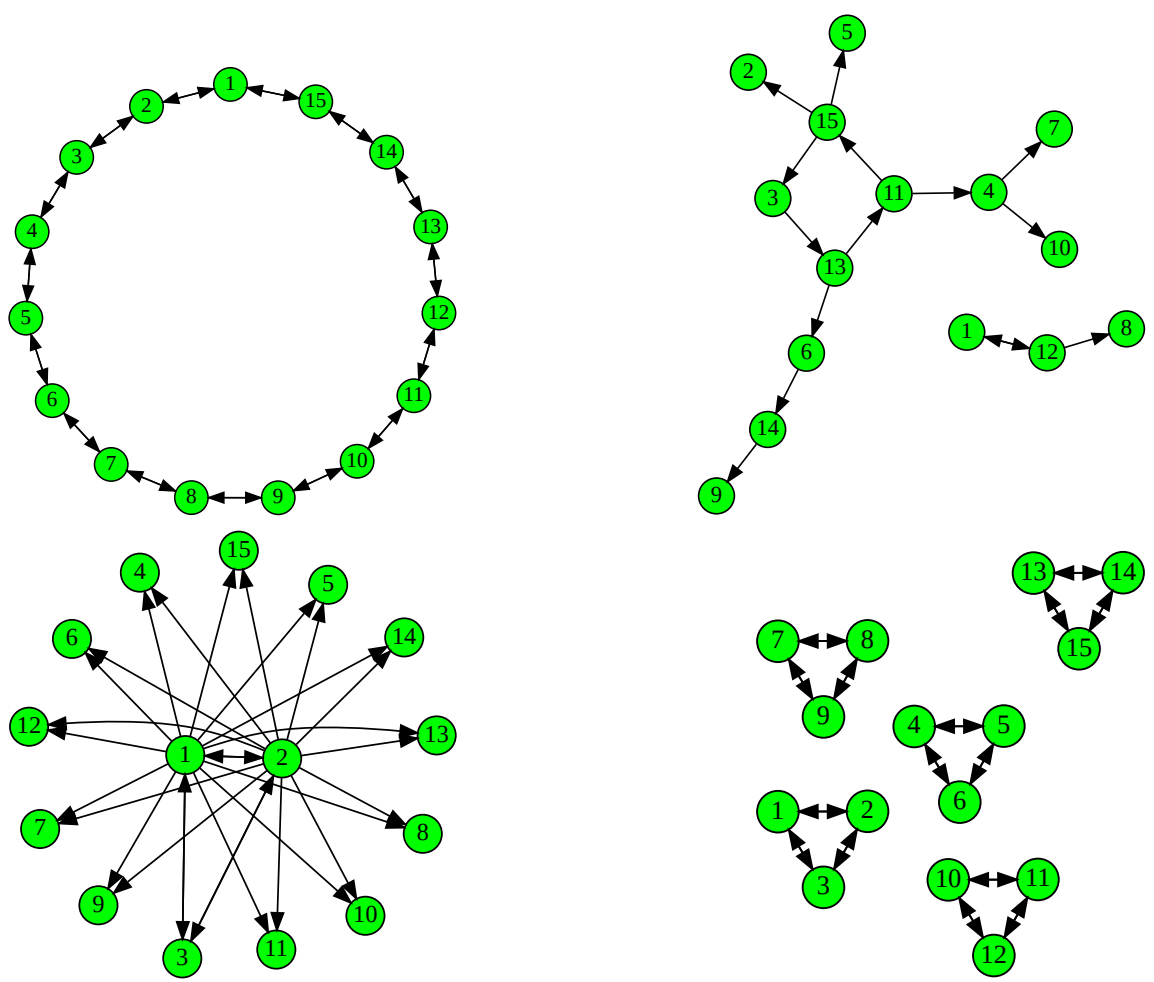

Fig. 2 Simplified NK structures for the examples shown in Fig. 1. Nodes represent loci and an arrow from one locus to another implies that the fitness contribution of the destination is dependent on the state of the source. In addition all locus contributions are dependent on their own state, but the resulting mandatory loops are not depicted. With $k=2$ the uRN structure forms components consisting of a single cycle with tree appendages.

for classical structures, and takes the universal form ${ }^{1}$

$$
\rho(d)=\frac{(L-k) !(L-d) !}{L !(L-k-d) !} .
$$

The corresponding Fourier spectrum was computed in [50].

It is sometimes useful to consider the incidence matrix of the structure hypergraph, i.e. the matrix with elements $b_{l, r} \in\{0,1\}$, where $l \in \mathcal{L}$ and $r \in\{1 \ldots|\mathcal{B}|\}$ and $b_{l, r}=1$ if and only if $l \in B_{r}$. As a measure of the structuredness of an NK structure the rank defined as

$$
r(\mathcal{B}) \equiv\left|\bigcup_{i=1}^{|\mathcal{B}|} \wp\left(B_{i}\right)\right|
$$

\footnotetext{
1 Note that incorrect expressions for $\rho(d)$ appear in some of the literature preceding 8 .
} 


\begin{tabular}{l|l|l} 
NK structure & Condition & Rank \\
\hline BN & exact & $\frac{L}{k}\left(2^{k}-1\right)+1$ \\
uRN & $L \gg k$ & $L\left(2^{k}-k\right)+1$ \\
AN & $L \geq 2 k-1$ & $L 2^{k-1}+1$ \\
SN & exact & $(L-k+2) 2^{k-1}$
\end{tabular}

Table 1 Ranks for some classical NK structures. Results are taken from 54 except for SN. The values for $\mathrm{uRN}$ and $\mathrm{BN}$ are the largest and smallest possible ones for classical structures [6].

has been introduced [6,54]. It is equal to the number of nonzero coefficients in the Fourier expansion Eq. (11). The ranks for some of the specific NK structures that will be discussed in the next subsection are listed in Table 1 .

\subsection{Specific structure choices}

So far no specific NK structure choice was made. In this subsection we introduce a few common, for the most part classical, NK structure choices.

- In the block neighborhood (BN) (with $L$ being an integer multiple of k) $\mathcal{L}$ is divided into $\frac{L}{k}$ disjoint $k$-subsets and the simplified structure graph is the union of complete symmetric graphs on each of these subsets 66. Each block effectively behaves as an independent HoC landscape. In contrast to the general case, analytical calculations are thus relatively simple, provided that the properties of the HoC model are already known, e.g. for the number of local maxima and the number of accessible pathways 60,66, 78. The BN is uniform, regular and classical.

- In the adjacent neighborhood (AN), loci are put on a circle and NK edges are given by the $k-1$ nearest neighbors of each locus on this ring. This is one of Kauffman's original choices. Similar to the BN, the AN is uniform, classical and regular. In contrast to the BN there is however no independence between subsets of loci.

- In the random neighborhood (RN), each classical NK structure is chosen with uniform probability. This structure is generally neither uniform, nor regular. In the uniform random neighborhood (uRN), each uniform classical NK structure is chosen with uniform probability. In the regular random neighborhood ( $\mathrm{rRN}$ ), each regular classical NK structure is chosen with uniform probability. In the uniform, regular random neighborhood ( $\mathrm{urRN}$ ), each uniform and regular classical NK structure is chosen with uniform probability. The last three modifications of the RN structure limit the space of possible structures to choose from. The random variant as used by Kauffman et al. [37,92], is actually our uRN. We expect all four variants to behave similarly, at least for large $k$, as the variation in regularity and uniformity will naturally shrink with increasing $k$.

- In the star neighborhood (SN) $k-1$ loci are chosen as center loci and they are contained in every block $B_{l}$. The other $L-k+1$ loci are 
called ray loci. A block $B_{l}$ associated with a ray locus contains the locus itself along with the $k-1$ center loci. When $l$ is a center locus, the remaining ( $k^{\prime}$ th) element in $B_{l}$ is set to one of the ray loci (but the same for each center locus). We introduce this structure as a stark contrast to the other models described above [77. While it too is classical and uniform, it is strongly non-regular. The center loci are present in $L$ NK edges giving them correspondingly large variances in mutational effects, while all other loci are only contained in a single NK edge. Furthermore distances in this structure are very small. Each pair of loci is in at most distance 2 along the structure hypergraph, while for all other models described above, the average distance between loci scales with $L$ at constant $k$. These differences will result in qualitatively different behavior of properties discussed later on. Note however that the distance autocorrelation function Eq. (15) is the same for the SN structure as for all other classical structures at equal $k$ and $L$.

- The mean field structure (MF) is not classical, containing each possible uniform edge exactly once. It is thus uniform and also regular. We use this mean field model as a slight variation from the original structures but with nice mathematical properties. Effectively we are distributing the average interaction strength of NK edges over all possible choices of these edges.

\section{Local fitness maxima}

In this section, we begin by introducing a general formalism for calculating the number of local maxima \# $\#_{\max }$ that can be applied to any of the (generalized) NK structures considered in this review. The primary goal of this formalism is to estimate the exponential growth rate $\lambda_{k}^{\text {model }}$ defined by the relation $\#_{\max } \sim\left(2 \lambda_{k}^{\text {model }}\right)^{L}$. The factor 2 is conventionally introduced in the literature simply to express the fact that the number of genotypes in the hypercube $\mathbb{H}_{\mathcal{L}}$ increases as $2^{L}$. Since the NK model is homogeneous, $\left(\lambda_{k}^{\text {model }}\right)^{L}$ may thus be interpreted as the probability that a randomly chosen genotype is a local maximum. As the number of fitness maxima cannot be smaller than 1 , the bounds $1 / 2 \leq \lambda_{k}^{\text {model }} \leq 1$ apply.

In order to minimize the notational burden unavoidable for the large degree of generalization to be pursued, we shall take a heuristic approach by starting with the HoC model as the simplest example and then extend our analysis to the NK model with arbitrary interaction structure. On this journey, we first encounter two exactly solvable cases, the block neighborhood (BN) and mean field (MF) models. Whereas the BN model was originally studied by Perelson and Macken [66, the MF model is introduced for the first time in the present work. In contrast to the strong universality hypothesis proposed by Weinberger 92 and cited above in Sec. 1.3 the distinct asymptotic behaviors exhibited by these two models exemplify our main finding that two different universal behaviors are realized depending on the choice of the NK structure.

To further investigate the range of possible behaviors, we then move our attention to two classical examples, the adjacent neighborhood (AN) and 
random neighborhood $(\mathrm{RN})$ structures. From our analysis of the AN model we recover most of the known exact results for $\lambda_{k}^{\mathrm{AN}}$ that were obtained previously [14,15,43. and subsequently extend these to a larger class of base distributions $p_{f}$. At the same time we strive to make the mathematical structure behind the formalism transparent to readers with a physics background, such as to enable them to more easily address future challenges in this field. Finally, we move on to a variant of the RN model where an exact solution for $\lambda_{k}^{\mathrm{RN}}$ can be obtained in the limit $k \rightarrow \infty$. Asymptotically we will find that $\lambda_{k}^{\mathrm{RN}}$ follows the same behavior as $\lambda_{k}^{\mathrm{MF}}$. Since the AN and BN models are known to display the same asymptotics, this implies that the AN and RN models are asymptotically distinct. For readers who want to get a quick overview of the results presented in this section a summary is provided in Sec. 3.3

\subsection{Number of local maxima for HoC fitness landscapes}

As explained above in Sec. 2.5. if we limit our interest to the mean number of local maxima, it is sufficient to pick an arbitrary reference genotype $\sigma$ and focus on the problem of finding the probability $\pi_{\max }$ for $\sigma$ being a local maximum. Once this is established, the total number of local maxima is trivially recovered by multiplying $\pi_{\max }$ by the number of genotypes $2^{L}$.

For the HoC model, following this procedure is quite straightforward: Because the fitness values of $\sigma$ and its neighbors are statistically independent and $F(\sigma)$ should be the largest among $L+1$ random variables, it is obvious that the probability $\pi_{\max }$ is $(L+1)^{-1}$ [35]. More detailed statistical properties of $\#_{\max }^{\mathrm{HoC}}$ can be found in [45,78.

However, for later purposes, let us forget this result for a moment and introduce a more general formalism for computing $\pi_{\max }$. Let $h_{0}$ and $h_{l}$ denote the fitness values of genotypes $\sigma$ and $\Delta_{l} \sigma$, respectively, i.e., $h_{0}=F(\sigma)$ and $h_{l}=F\left(\Delta_{l} \sigma\right)$. Then, $\sigma$ is a local maximum if $h_{0}>h_{l}$ or $u_{l} \equiv h_{0}-h_{l}>0$ for all $1 \leq l \leq L$. Using the vector notation $\mathbf{u} \equiv\left(u_{1}, u_{2}, \cdots, u_{L}\right)$, the joint probability density of the $u_{l}$ is given by

$$
\mathcal{P}(\mathbf{u})=\int \prod_{l=0}^{L} d h_{l} p_{f}\left(h_{l}\right) \prod_{l=1}^{L} \delta\left(u_{l}-\left(h_{0}-h_{l}\right)\right),
$$

or alternatively, the characteristic function reads

$$
\begin{aligned}
\Phi(\mathbf{q}) & =\int \prod_{l=1}^{L} d u_{l} e^{i \sum_{l=1}^{L} q_{l} u_{l}} \mathcal{P}(\mathbf{u})=\int \prod_{l=0}^{L} d h_{l} p_{f}\left(h_{l}\right) e^{i \sum_{l=1}^{L} q_{l}\left(h_{0}-h_{l}\right)} \\
& =\phi_{f}\left(\sum_{l=1}^{L} q_{l}\right) \prod_{l=1}^{L} \phi_{f}\left(-q_{l}\right)=\int d y p_{f}(y) \prod_{l=1}^{L} \phi_{f}\left(-q_{l}\right) \exp \left(i y \sum_{l=1}^{L} q_{l}\right)
\end{aligned}
$$

where $\phi_{f}(q)$ is the characteristic function of $p_{f}(h)$. By performing the inverse Fourier transform of $\Phi(\mathbf{q})$ and then integrating over only positive values of 
$u_{l}$, we obtain

$$
\pi_{\max }=\int_{0}^{\infty} \prod_{l=1}^{L} d u_{l} \mathcal{P}(\mathbf{u})=\int \frac{\mathcal{D} \mathbf{u} \mathcal{D} \mathbf{q}}{(2 \pi)^{L}} e^{-i \mathbf{u} \cdot \mathbf{q}} \Theta(\mathbf{u}>0) \Phi(\mathbf{q})
$$

where we have introduced a symbol $\mathcal{D}$ to denote the integration over $L$ dimensional real space (i.e., $\mathcal{D} \mathbf{v}=\prod_{l=1}^{L} d v_{l}$ ). Moreover, to encode the positivity condition for $\mathbf{u}$, we define the theta function $\Theta(\mathbf{u}>0)$ such that it is one if all the elements of $\mathbf{u}$ are positive and zero otherwise.

Now, we are ready to calculate $\pi_{\max }$. Inserting Eq. $(18)$ into Eq. 19 and making use of the integral representation of the delta function

$$
\delta(q)=\int \frac{d y}{2 \pi} e^{i y q}
$$

leads us to write

$$
\begin{aligned}
\pi_{\max } & =\int d y p_{f}(y) \int \frac{\mathcal{D} \mathbf{u} \mathcal{D} \mathbf{q}}{(2 \pi)^{L}} e^{-i \mathbf{u} \cdot \mathbf{q}} \Theta(\mathbf{u}>0) \prod_{l=1}^{L} \phi_{f}\left(-q_{l}\right) e^{i q_{l} y} \\
& =\int d y p_{f}(y)\left[\int_{0}^{\infty} d u p_{f}(y-u)\right]^{L}
\end{aligned}
$$

Finally, by realizing that $G(y)=\int_{0}^{\infty} d m p_{f}(y-m)=\int_{-\infty}^{y} d y p_{f}(y)$ is the cumulative base distribution, the substitution $x=G(y)$ is evaluated to

$$
\pi_{\max }^{\mathrm{HoC}}=\int_{0}^{1} d x x^{L}=\frac{1}{L+1},
$$

which is the desired result for the HoC model. The fact that $\pi_{\max }^{\mathrm{HoC}}$ decays algebraically in $L$ implies $\lambda_{k}^{\mathrm{HoC}}=1$.

3.2 Number of local maxima for NK fitness landscapes

By the construction of the NK model as described in Eq. $(12)$, the fitness $F(\sigma)$ of a sequence $\sigma$ is the sum of HoC fitness values defined on the subspaces $\mathbb{H}_{B_{r}}$ spanned by the edge sets or NK blocks $B_{r}$. Since a characteristic function is a natural object when dealing with a random quantity constructed from the sum of independent random variables, we will build our approach upon the characteristic functions of the NK blocks. Specifically, we expect the characteristic function of $\mathbf{u}$ to be of the form

$$
\Phi(\mathbf{q})=\prod_{r=1}^{|\mathcal{B}|} \Phi_{r}(\mathbf{q})
$$

where $\Phi_{r}(\mathbf{q})$ denotes the characteristic function of $\mathbf{u}$ within the NK block $B_{r}$. Because each $\mathrm{HoC}$ model is defined only on a subset of $\mathcal{L}$, it is convenient 
to employ the incidence matrix notation $b_{l, r}$ that indicates the presence (absence) of a locus $l$ in a neighborhood set $r$, i,e, $b_{l, r}=1(0)$ if $l \in B_{r}\left(l \notin B_{r}\right)$. In terms of these variables, the characteristic function $\Phi_{r}$ can be rewritten in the following form:

$$
\begin{aligned}
\Phi_{r}(\mathbf{q}) & =\phi_{f}\left(\sum_{l=1}^{L} q_{l} b_{l, r}\right) \prod_{l=1}^{L} \phi_{f}\left(-q_{l}\right)^{b_{l, r}} \\
& =\int d y_{r} p_{f}\left(y_{r}\right) \prod_{l=1}^{L}\left[\phi_{f}\left(-q_{l}\right) e^{i y_{r} q_{l}}\right]^{b_{l, r}} .
\end{aligned}
$$

Once the full characteristic function Eq. 23) has been derived, $\pi_{\max }$ is readily calculated by inverse Fourier transform along the lines of Eq. (19), i.e.,

$$
\pi_{\max }=\int \frac{\mathcal{D} \mathbf{u} \mathcal{D} \mathbf{q}}{(2 \pi)^{L}} e^{-i \mathbf{u} \cdot \mathbf{q}} \Theta(\mathbf{u}>0) \int \mathcal{D} \mathbf{y} \mathcal{P}(\mathbf{y}) \prod_{r=1}^{|\mathcal{B}|} \prod_{l=1}^{L}\left[\phi_{f}\left(-q_{l}\right) e^{i y_{r} q_{l}}\right]^{b_{l, r}}
$$

where $\mathcal{P}(\mathbf{y})=\prod_{r} p_{f}\left(y_{r}\right)$, the $|\mathcal{B}|$-dimensional base fitness distribution.

Below we will follow these steps to compute $\pi_{\max }$ for several known NK structures as well as for the MF structure introduced in Sec. 2.7. By doing so, we will recover earlier results and obtain new insights into how the universal and non-universal behavior of $\pi_{\max }$ is shaped by the interaction structure and the base fitness distribution.

\subsubsection{Block neighborhood}

In the $\mathrm{BN}$ model, the $\mathrm{NK}$ structure $\mathcal{B}$ comprises mutually non-overlapping sets of size $k$. Each $B_{r}$ thus defines an independent module in which the loci are correlated among each other but not with the loci outside of the module. This non-overlapping property facilitates the analysis dramatically since it allows us to write $\pi_{\max }$ in a factorized form, $\pi_{\max }^{\mathrm{BN}}=\prod_{r} \pi_{\max }^{r}$ where $\pi_{\max }^{r}$ is simply $\pi_{\max }^{\mathrm{HoC}}$ for $k$ loci, as given by Eq. 22, Putting everything together, we find

$$
\pi_{\max }^{\mathrm{BN}} \equiv\left(\lambda_{k}^{\mathrm{BN}}\right)^{L}=\prod_{r=1}^{|\mathcal{B}|} \frac{1}{k+1}=\left(\frac{1}{k+1}\right)^{L / k}
$$

where we have used the fact that the number of blocks is $L / k$. Equivalently, the mean number of local maxima is

$$
\mathbb{E}\left[\#_{\max }^{\mathrm{BN}}\right]=2^{L}\left(\frac{1}{k+1}\right)^{L / k}
$$

As consistency checks, one can immediately show that inserting $k=1$ and $k=L$ recovers $\mathbb{E}\left[\#_{\max }^{\mathrm{BN}}\right]=1$ for additive landscapes and $\mathbb{E}\left[\#_{\max }^{\mathrm{BN}}\right]=(L+$ $1)^{-1} 2^{L}$ for HoC landscapes, respectively. 
This closed form solution allows us to study the asymptotic behaviors in various limits. The most interesting scaling limits include i) $L \rightarrow \infty$ for $k$ fixed and ii) the joint limit $L, k \rightarrow \infty$ with fixed $\alpha=k / L$. In the first limit, it is clear that $\pi_{\max }^{\mathrm{BN}}$ increases exponentially with $L$ with an exponential growth rate

$$
\ln \lambda_{k}^{\mathrm{BN}} \equiv \lim _{L \rightarrow \infty} \frac{\ln \pi_{\max }^{\mathrm{BN}}}{L}=\ln \left(\frac{1}{k+1}\right)^{1 / k}=-\frac{\ln k}{k}+O\left(\frac{1}{k^{2}}\right)
$$

as $L \rightarrow \infty$. As $k \rightarrow \infty, \ln \lambda_{k}^{\mathrm{BN}}$ converges to the theoretical upper bound, namely zero. Thus, for larger $k$, we expect more rugged fitness landscapes.

In such a large $k$ limit, the second scaling limit, where $\alpha$ is kept fixed, provides a better understanding of the behavior of $\pi_{\mathrm{max}}^{\mathrm{BN}}$. In this limit, it is evident that the leading exponential behavior of $\mathbb{E}\left[\#_{\max }^{\mathrm{BN}}\right]$ should be $2^{L}$. The correction to this exponential behavior should be at most algebraic as already seen in the $\mathrm{HoC}$ model. In the case of $\mathrm{BN}$, this correction may be easily evaluated to

$$
\pi_{\max }^{\mathrm{BN}}=\left(\frac{1}{L \alpha}+O\left(L^{-2}\right)\right)^{1 / \alpha} \sim L^{-1 / \alpha}
$$

A more detailed analysis of the BN model has been conducted in the literature [66, 78, and in particular, the second moment of $\#_{\max }^{\mathrm{BN}}$ is given by

$$
\mathbb{E}\left[\left(\#_{\max }^{\mathrm{BN}}\right)^{2}\right]=\left(\mathbb{E}\left[\#_{\max }^{\mathrm{BN}}\right]\right)^{2}\left(1+\frac{k-1}{2^{k+1}}\right)^{L / k} .
$$

\subsubsection{MF neighborhood}

The mean-field NK structure is another extreme type of NK model. In this case the neighborhood set $\mathcal{B}$ contains all possible subsets of size $k$, which effectively makes the fitness landscape unstructured in contrast to the block model which has a well-defined modular structure. By construction, the size of $\mathcal{B}$ is given by $|\mathcal{B}|=\left(\begin{array}{l}L \\ k\end{array}\right)$ unlike classical NK structures that satisfy $|\mathcal{B}|=L$. Because of this huge combinatorial factor, one might wonder if an additional normalization that rescales the overall fitness to a reasonable level should be introduced. While this might be necessary for other applications, we do not bother with it here since the number of local maxima only depends on the fitness ordering between neighboring genotypes and not on the overall fitness scale.

Additionally, we assume that the base fitness distribution $p_{f}(h)$ is a standard Gaussian distribution. This assumption is made for two reasons. First of all, the choice of Gaussian distribution greatly simplifies the analysis of \# $_{\text {max }}^{\mathrm{MF}}$. Secondly and more importantly, the number of local maxima \# $_{\max }^{\mathrm{RN}}$ for the RN NK structure complemented by a large class of base fitness distributions will be shown to follow the same limiting behavior as $\#_{\text {max }}^{\mathrm{MF}}$. Verifying this claim for universal behavior will be the main topic of Sec. 3.2.4. 
Recalling the fact that $\phi_{f}(q)=e^{-q^{2} / 2}$ for a standard Gaussian distribution and using the first identity in Eq. 24), the characteristic function is readily obtained as

$$
\Phi^{\mathrm{MF}}(\mathbf{q})=\exp \left(-\sum_{l}\left(\begin{array}{l}
L-1 \\
k-1
\end{array}\right) q_{l}^{2}-\sum_{l>m}\left(\begin{array}{l}
L-2 \\
k-2
\end{array}\right) q_{l} q_{m}\right) .
$$

The two binomial numbers correspond to the number of neighborhood sets that contain the locus $l$, and both the loci $l$ and $m$, respectively.

Now, we are left to calculate $\pi_{\max }^{\mathrm{MF}}$ using Eq. 19p. As mentioned before, any rescaling of fitness values should leave the quantity of interest unchanged. Exploiting this invariance, the fitness rescaling $F(\sigma) \rightarrow F(\sigma) / \sqrt{\left(\begin{array}{l}L-2 \\ k-2\end{array}\right)}$ allows the subsequent transformations $u_{l} \rightarrow u_{l} / \sqrt{\left(\begin{array}{l}L-2 \\ k-2\end{array}\right)}$ and $q_{l} \rightarrow \sqrt{\left(\begin{array}{l}L-2 \\ k-2\end{array}\right)} q_{l}$, which effectively reduces the number of free parameters to one. Defining

$$
\eta=\sqrt{\frac{2(2 L-k-1)}{L(k-1)}}
$$

the probability reads

$$
\pi_{\max }^{\mathrm{MF}}=\int \frac{\mathcal{D} \mathbf{u} \mathcal{D} \mathbf{q}}{(2 \pi)^{L}} e^{-i \mathbf{u} \cdot \mathbf{q}} \Theta(\mathbf{u}>0) \exp \left[-\sum_{l=1}^{L} \frac{L \eta^{2}}{4} q_{l}^{2}-\frac{1}{2}\left(\sum_{l=1}^{L} q_{l}\right)^{2}\right]
$$

Finally, employing the Hubbard-Stratonovich transform, the quadratic coupling term in the square bracket is linearized and the integrals for different indices $l$ are completely decoupled:

$$
\begin{aligned}
\pi_{\max }^{\mathrm{MF}} & =\sqrt{\frac{L}{2 \pi}} \int d y e^{-L y^{2} / 2}\left[\frac{1}{2 \pi} \int_{0}^{\infty} d u \int d q e^{-\frac{L \eta^{2}}{4} q^{2}-i q u+i \sqrt{L} y q}\right]^{L} \\
& =\sqrt{\frac{L}{2 \pi}} \int d y e^{-L y^{2} / 2}\left[\frac{1}{2}\left(\operatorname{erf}\left(\frac{y}{\eta}\right)+1\right)\right]^{L}=\sqrt{\frac{L}{2 \pi}} \int d y e^{L \mathcal{F}_{\eta}(y)},
\end{aligned}
$$

where

$$
\mathcal{F}_{\eta}(y)=-y^{2} / 2+\ln \left[\frac{1}{2}\left(\operatorname{erf}\left(\frac{y}{\eta}\right)+1\right)\right] .
$$

The integral in Eq. (34) does not allow for a closed form solution for general $\eta$. However, a straightforward calculation shows that the expected results can be recovered in the two limiting cases $k=1$ (for linear landscapes) and $k=L$ (for HoC landscapes).

To proceed, a reasonable scaling limit should be taken to draw some practical conclusions. Let us first consider the large $L$ limit with fixed $k$, which was discussed above for the BN model. In this limit, the parameter in Eq. 32 is expanded as $\eta^{2}=\frac{4}{k-1}+O(1 / L)$. The fact that $\eta$ is independent of 

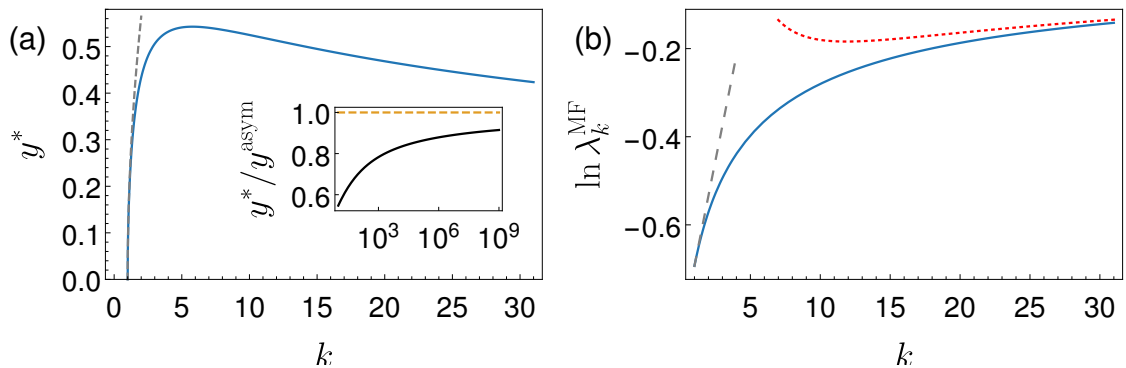

Fig. 3 Plots of $y^{*}$ and $\ln \lambda_{k}^{\mathrm{MF}}$ as a function of $k$. (a) The solid curve indicates the numerical solution for $y^{*}$. The dashed curve shows the first order approximation $\sqrt{\frac{k-1}{\pi}}$ in Eq. 37 as $k \rightarrow 1$. (inset) The black curve represents the ratio of $y^{*}$ to the first order approximation $y^{\text {asymp }}=2 \sqrt{\frac{\log (k)}{k}}$, which is valid for $k \rightarrow \infty$. It illustrates the slow convergence of $y^{*}$ to the first order approximation even for relatively large $k$, i.e., $k \sim 10^{9}$. (b) The solid line describes the actual value of $\ln \lambda_{k}^{\mathrm{MF}}$ found numerically from the variational problem. Two dashed curves are obtained by the first order approximations for both limits, i.e., $\frac{k-1}{2 \pi}-\ln (2)$ for $k \rightarrow 1$ and Eq. 39 for $k \rightarrow \infty$.

$L$ up to leading order in $L$ suggests that the integral may be evaluated using the saddle point method up to a correction of $O(1 / L)$. We point out that once the value $y^{*}$ that maximizes the "action" Eq. (35) has been found, the value of $\lambda_{k}^{\mathrm{MF}}$ readily follows from $\ln \lambda_{k}^{\mathrm{MF}}=\mathcal{F}_{\eta}\left(y^{*}\right)$. Specifically, assuming $y^{*}$ is known, the saddle point approximation yields a rather formidable formula:

$$
\pi_{\max }^{\mathrm{MF}}=\frac{\left(\frac{e^{-\frac{\left(y^{*}\right)^{2}}{2}}}{2}\left(\operatorname{erf}\left(\frac{y^{*} \sqrt{k-1}}{2}\right)+1\right)\right)^{L} \exp \left(\frac{\sqrt{k-1}(k+1) y^{*} e^{-\frac{1}{4}(k-1)\left(y^{*}\right)^{2}}}{4 \sqrt{\pi}\left(\operatorname{erf}\left(\frac{y^{*}}{2} \sqrt{k-1}\right)+1\right)}\right)}{\sqrt{\frac{(k-1)^{3 / 2} y^{*} e^{-\frac{1}{4}(k-1)\left(y^{*}\right)^{2}}}{2 \sqrt{\pi}\left(\operatorname{erf}\left(\frac{y^{*}}{2} \sqrt{k-1}\right)+1\right)}+\frac{(k-1) e^{-\frac{1}{2}(k-1)\left(y^{*}\right)^{2}}}{\pi\left(\operatorname{erf}\left(\frac{y^{*}}{2} \sqrt{k-1}\right)+1\right)^{2}}+1}}
$$

with an error of the order $O\left(L^{-1}\right)$.

Even though the variational problem has no closed form solution in general, one can analyze the asymptotic series expansion for small or large $\eta$ as

$$
y^{*}=\left\{\begin{array}{lll}
\frac{2}{\sqrt{\pi} \eta}+O\left(\eta^{-2}\right) & \text { for } & \eta \rightarrow \infty \\
\sqrt{-2 \eta^{2} \ln \eta+O(\ln (|\ln \eta|))} & \text { for } & \eta \rightarrow 0 .
\end{array}\right.
$$

Subsequently, this expansion allows us to obtain

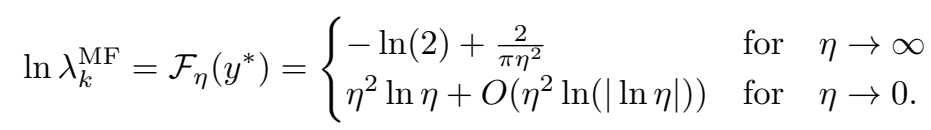

With the results written in terms of $\eta$, the functional dependence on $k$ can be easily recovered by the relation $\eta^{-2}=\frac{k-1}{4}$ as defined in Eq. 32. The 
saddle point $y^{*}$ that maximizes $\mathcal{F}_{\eta}(y)$ and the corresponding exponential factor $\ln \lambda_{k}^{\mathrm{MF}}$ are illustrated in Fig. 3 as a function of $k$.

The small $\eta$ expansion in Eq. 38 translates into the expression

$$
\ln \lambda_{k}^{\mathrm{MF}} \approx \frac{\ln (16)-2 \ln (k-1)}{k-1}=-\frac{2 \ln (k)}{k}+O\left(\frac{1}{k}\right)
$$

which is noteworthy for two reasons. First, and most importantly, the leading order behavior $\ln \lambda_{k}^{\mathrm{MF}} \sim-\frac{2 \ln (k)}{k}$ differs from that obtained for the block model, $\ln \lambda_{k}^{\mathrm{BN}} \sim-\frac{\ln (k)}{k}$, which contradicts the claim of universality originally stated by Weinberger [92]. Second, the leading term in Eq. (39) is only logarithmically larger than the next-to-leading term. Thus, in the range of $k$ that is accessible to the explicit numerical evaluation of \# $\#_{\max }$ for arbitrary NK structures, i.e., at most $k \sim O\left(10^{2}\right)$, the next-to-leading correction remains substantial. Nevertheless, the full expression in Eq. (39) provides an accurate approximation to the true behavior already for $k=30$ [see Fig. 3 (b)].

Although the calculation as described relies on taking the limit $L \rightarrow \infty$ before the limit of large $k$, extending the result to the joint limit $L, k \rightarrow \infty$ at fixed $\alpha=k / L$ is straightforward at least on a formal level. For this it suffices to note that Eq. 32 now implies the relation $\eta \approx \frac{2}{\sqrt{k}} \sqrt{1-\frac{\alpha}{2}}$, which combined with the $\eta \rightarrow 0$ limit in Eq. (38) yields

$$
\ln \lambda_{k}^{\mathrm{MF}} \approx-\frac{(2-\alpha) \ln k}{k} \text { and } \pi_{\max }^{\mathrm{MF}} \sim L^{-\left(\frac{2}{\alpha}-1\right)}
$$

The exponent of the algebraic decay of $\pi_{\max }$ is different from that obtained in Eq. (29) for the BN structure, but reduces to the $1 / L$-behavior expected for the HoC model when $\alpha \rightarrow 1$. A rigorous analysis based on extreme value theory confirms this simple argument up to logarithmic corrections (Appendix A).

As we will see in the following, the different asymptotics obtained for the $\mathrm{BN}$ and $\mathrm{MF}$ models are not just arbitrary examples created by unusual choices of NK structures, but in fact they appear to be robust across large classes of structures. They exemplify a somewhat surprising trend, which is that NK models with more structured interaction schemes such as the BN model result in more rugged fitness landscapes. In the next two subsections we will explore two other NK structures, each of which follows the asymptotic behavior found for the BN and MF models, respectively.

\subsubsection{Adjacent neighborhood}

The regularity of the AN NK structure allows us to view our analysis from a different angle. This point is best described by Eq. (25) with a slight mod- 
ification given as

$$
\begin{aligned}
\pi_{\max }^{\mathrm{AN}} & =\int \mathcal{D} \mathbf{y} \mathcal{P}(\mathbf{y}) \frac{\mathcal{D} \mathbf{u} \mathcal{D} \mathbf{q}}{(2 \pi)^{L}} e^{-i \mathbf{u} \cdot \mathbf{q}} \Theta(\mathbf{u}>0) \prod_{r=1}^{L} \prod_{l=1}^{L}\left[\phi_{f}\left(-q_{l}\right) e^{i y_{r} q_{l}}\right]^{b_{l, r}} \\
& =\int \mathcal{D} \mathbf{y} \mathcal{P}(\mathbf{y}) \frac{\mathcal{D} \mathbf{u} \mathcal{D} \mathbf{q}}{(2 \pi)^{L}} e^{-i \mathbf{u} \cdot \mathbf{q}} \Theta(\mathbf{u}>0) \prod_{l=1}^{L} \phi_{f}\left(-q_{l}\right)^{k} e^{i q_{l} \sum_{r=0}^{k-1} y_{(l+r) \bmod L}},
\end{aligned}
$$

where the operator $A \bmod B$ is used to denote the remainder of $A$ when divided by $B$. Also, it is worth pointing out that the characteristic function for each locus $l$ always appears $k$ times due to the translational invariance. Since the $k$-th power of a characteristic function is Fourier-transformed back to the $k$-th convolution of the corresponding probability density, the integrals for $\mathbf{u}$ and $\mathbf{q}$ may be written in terms of $\tilde{F}^{(k)}(z)=\int_{-\infty}^{z} d y p_{f}^{(k)}(y)$, where $p_{f}^{(k)}(y)$ is the $k$-fold convolution of $p_{f}(y)$ :

$$
\pi_{\max }^{\mathrm{AN}}=\int \mathcal{D} \mathbf{y} \mathcal{P}(\mathbf{y}) \prod_{l=1}^{L} \tilde{F}^{(k)}\left(\sum_{r=0}^{k-1} y_{(l+r) \bmod L}\right) .
$$

This elegant equation was first derived by Weinberger 92. To understand this expression better, it is convenient to expand the product for the simplest case $k=2$. Then, one may identify a simple pattern of the following form

$$
\pi_{\max }^{\mathrm{AN}}=\int \prod_{r=1}^{L} d y_{r} K_{w}\left(y_{1} ; y_{2}\right) K_{w}\left(y_{2} ; y_{3}\right) \cdots K_{w}\left(y_{L} ; y_{1}\right)
$$

where

$$
K_{w}(x ; y) \equiv p_{f}(x)^{w} \tilde{F}^{(2)}(x+y) p_{f}(y)^{1-w},
$$

with an arbitrary choice of $w \in[0,1]$. Thus, $\pi_{\max }^{\mathrm{AN}}$ may be regarded as the trace of the $L$-th power of an integral operator defined by the integral kernel $K_{w}(x ; y)$. One can show that the eigenvalue spectrum of the kernel does not depend on the choice of $w$ by checking that the trace of an arbitrary power of $K_{w}(x ; y)$ is independent of $w$. Moreover, the fact that $K_{w}(x ; y)$ becomes symmetric when $w=1 / 2$ guarantees that all the eigenvalues of this operator are real.

This construction recasts the problem of finding $\pi_{\max }^{\mathrm{AN}}$ into an eigenvalue problem for the integral kernel $K_{w}(x ; y)$. In particular, the largest eigenvalue will correspond to $\lambda_{2}^{\mathrm{AN}}$ in the limit $L \rightarrow \infty$. A similar but not identical transfer matrix technique for $\pi_{\max }^{\mathrm{AN}}$ was originally introduced by Evans and Steinsaltz [15].

Finding eigenvalues of arbitrary integral operators is in general a nontrivial problem [34. However, if $K_{w}(x ; y)$ is separable, i.e., if $K_{w}(x ; y)$ can be cast into a sum of factorized terms of the form

$$
K_{w}(x ; y)=\sum_{p=1}^{n} u_{p}(x) v_{p}(y)
$$


the problem can be mapped to finding the eigenvalues of an $n \times n$ matrix with matrix elements given by

$$
T_{p q}=\int d x u_{p}(x) v_{q}(x)
$$

In the following, we will provide two classes of base distributions that allow for an exact solution through this technique.

As the simplest example, let us consider a random variable $Z$ with the property that $\tilde{F}^{(2)}(z)=1-e^{-z}$ for $0 \leq z \leq \infty$. In other words, the twofold convolution of the base probability density is exponential. From the definition of the integral kernel Eq. (45) with the choice of $w=1$, one finds that $K_{1}(x ; y)=p_{f}(x)-p_{f}(x) e^{-x} e^{-y}$. The corresponding matrix is readily obtained as

$$
T=\left(\begin{array}{cc}
1 & \left.\mathbb{E}\left[e^{i k X}\right]\right|_{k=i} \\
-\left.\mathbb{E}\left[e^{i k X}\right]\right|_{k=i} & -\left.\mathbb{E}\left[e^{i k X}\right]\right|_{k=2 i}
\end{array}\right)=\left(\begin{array}{cc}
1 & \frac{1}{\sqrt{2}} \\
-\frac{1}{\sqrt{2}} & -\frac{1}{\sqrt{3}}
\end{array}\right),
$$

where we have calculated the characteristic function of $X$ to be $\sqrt{\frac{i}{k+i}}$ by taking the square root of the characteristic function of the exponential distribution. Finally, we can easily calculate the largest eigenvalue as $\lambda_{2}^{\mathrm{AN}}=$ $\frac{1}{6}(3-\sqrt{3}+\sqrt{6 \sqrt{3}-6}) \simeq 0.560622$, a result originally derived in [54].

The base fitness distribution corresponding to the previous example is a gamma distribution with shape parameter $s=1 / 2$, and in fact gammadistributed fitness values appear in several earlier studies where exact results for $\lambda_{2}^{\text {AN }}$ were obtained [14,15. With the current framework at hand, it turns out that the association of solvable instances of the AN model with certain gamma distributions is not a coincidence. Below we will show that the integral kernels $K_{w}(x ; y)$ generated by gamma distributions with shape parameter $s$ being either a half-integer or an integer are separable and thus all the previously known results can be calculated in a uniform manner.

For an arbitrary shape parameter $s$, the two quantities defining $K_{w}(x ; y)$ in Eq. (44) are given by

$$
p_{f}(z)=g_{s}(z) \equiv \frac{e^{-z} z^{s-1}}{\Gamma(s)}
$$

and

$$
\tilde{F}_{s}^{(2)}(z)=1-\frac{\Gamma(2 s, z)}{\Gamma(2 s)} .
$$

Furthermore, the incomplete gamma function permits a series expansion of length $2 s$,

$$
\Gamma(2 s, z)=\Gamma(2 s) e^{-z} \sum_{m=0}^{2 s-1} \frac{z^{m}}{m !}
$$


provided $2 s$ is an integer. Inserting this into Eq. (44), we arrive at

$$
K_{1}(x ; y)=-\sum_{p=0}^{2 s-1} \frac{e^{-2 x} x^{p+s-1}}{\Gamma(s)} \times \frac{\Gamma(2 s-p, y)}{p ! \Gamma(2 s-p)}+g_{s}(x) .
$$

When cast into the form of Eq. 45), this shows that the integral operator $K_{1}(x ; y)$ is mapped onto a $(2 s+1) \times(2 s+1)$ matrix with entries given by

$$
T_{p q}=\left\{\begin{array}{ccc}
-M_{p q} & \text { if } \quad 0 \leq p, q \leq(2 s-1) \\
L_{p} & \text { if } q=2 s, 0 \leq q \leq(2 s-1) \\
-R_{q} & \text { if } \quad p=2 s, 0 \leq p \leq(2 s-1) \\
1 & \text { if } \quad p=2 s, q=2 s
\end{array},\right.
$$

where

$$
\begin{aligned}
M_{p q}= & \int_{0}^{\infty} d x \frac{e^{-2 x} x^{p+s-1}}{\Gamma(s)} \times \frac{\Gamma(2 s-q, x)}{q ! \Gamma(2 s-q)} \\
= & \frac{{ }_{2} F_{1}(p+s, p-q+3 s ; p+s+1 ;-2) \Gamma(p-q+3 s)}{q !(p+s) \Gamma(s) \Gamma(2 s-q)} \\
& L_{p}=\int_{0}^{\infty} d x \frac{e^{-2 x} x^{p+s-1}}{\Gamma(s)}=\frac{2^{-p-s} \Gamma(p+s)}{\Gamma(s)}
\end{aligned}
$$

and

$$
\begin{aligned}
R_{q} & =\int_{0}^{\infty} d x \frac{e^{-x} x^{s-1}}{\Gamma(s)} \times \frac{\Gamma(2 s-q, x)}{q ! \Gamma(2 s-q)} \\
& =\frac{{ }_{2} F_{1}(s, 3 s-q ; s+1 ;-1) \Gamma(3 s-q)}{\Gamma(q+1) \Gamma(s+1) \Gamma(2 s-q)} .
\end{aligned}
$$

Setting $s=1 / 2$ in Eq. (52), we immediately reproduce the transfer matrix obtained in the previous example Eq. 47). Note that since the gamma distribution converges to a Gaussian distribution as $s \rightarrow \infty, \lambda_{2}^{\mathrm{AN}}$ for the Gaussian distribution can be obtained by examining the asymptotic behavior for large $s$.

To provide a larger class of exactly solvable cases, one might hope that a similar approach can be taken for random variables $Y$ that are transformed from a gamma distributed random variable $X$, if the transformation function $Y=f(X)$ is sufficiently simple. One such example is $Y=-X$, and we call the corresponding distribution a negative gamma distribution. As a special case of this distribution, the value of $\lambda_{2}^{\mathrm{AN}}$ for $s=1$ has been found in [14. The structural similarity possessed by the transformed distribution allows us to repeat the same procedure that we followed for the gamma distribution. In this case, we find that the corresponding transfer matrix is of size $(2 s) \times(2 s)$ with matrix elements

$$
T_{p q}=M_{p q},
$$

where $M_{p q}$ was defined in Eq. (53). 

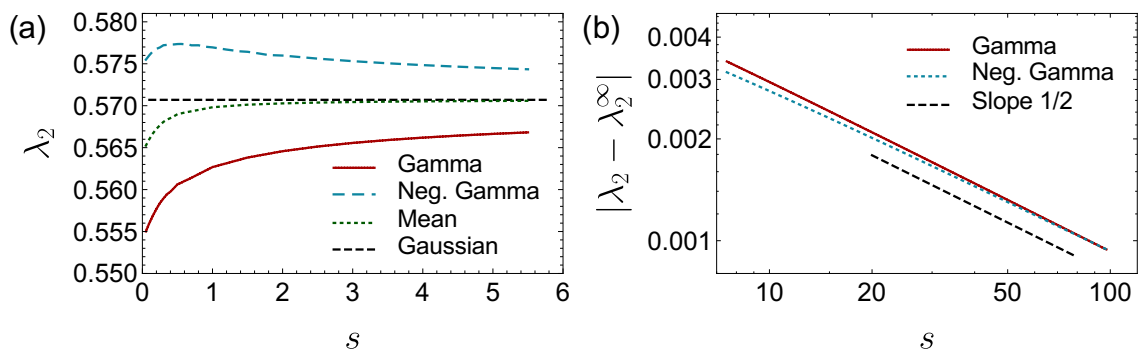

Fig. 4 (a) A plot of $\lambda_{2}^{\mathrm{AN}}$ as a function of the shape parameter $s$ for the gamma distribution (red solid line) and the negative gamma distribution (blue dashed line). For integer or half-integer values of $s$, the largest eigenvalue of the transfer matrix $T$ given by Eq. (52) and Eq. (56) is computed numerically while for the other values of $s$, estimates from numerical simulations are used. Since the convergence to the asymptotic exponential behavior is very rapid in this case, it was sufficient to use sequence lengths between $L=4$ and 7 for which the number of maxima could be determined by explicit enumeration. The average of the red solid curve and the blue dashed curve is represented by the green dotted curve. Within the resolution of the image, the average appears to quickly converge to $\lambda_{2}=0.5707$, the value numerically obtained for a Gaussian distribution as indicated by the black dashed line. (b) A double-logarithmic plot showing the speed of convergence for the gamma and the negative gamma distribution. The value of $\lambda_{2}^{\infty}$ is estimated from the average of the two curves using the largest shape parameter numerically available. The black dashed line is provided as a visual guide to show that the curves decay algebraically as $s^{-1 / 2}$.

Once the matrix has been set up according to Eq. (52) or Eq. (56), the largest eigenvalue is computed numerically through a standard algorithm. The behaviors of $\lambda_{2}^{\mathrm{AN}}$ for the gamma distribution and the negative gamma distribution are illustrated in Fig. 4. In particular, this shows that both curves converge algebraically as $s^{-1 / 2}$ to the value of the Gaussian distribution. Since the curve for the gamma distribution converges from below whereas the curve for the negative gamma distribution converges from above, the average of the two curves should provide an accurate estimate for the Gaussian distribution. In fact, we found that the sub-leading corrections for the two curves seem to perfectly cancel each other. Thus, one can see from the comparison with simulation results obtained for the sequence length $L=128$ (black dashed line of Fig. $4(\mathrm{a}))$, that a very precise estimate $\left(\lambda_{2}^{\mathrm{AN}}=0.5707\right)$ can be obtained even for relatively small $s$.

At this point, it is worth noticing that $\lambda_{2}^{\mathrm{AN}}$ for the negative gamma distribution is maximized at $s=1 / 2$. Since our analytical framework is only applicable for $s$ being either an integer or a half-integer, extensive simulations in the vicinity of $s=1 / 2$ had to be performed to create a smooth curve around $s=1 / 2$ in Fig. 4 (a). What is more interesting about this point is the fact that the eigenvalue problem becomes trivial, as $T$ becomes a $1 \times 1$ matrix with the single element $1 / \sqrt{3}$. Surprisingly, this number coincides with exact value of $\lambda_{2}^{\mathrm{BN}}$, as given by Eq. 28), for the case $k=2$. We show in Appendix B that the correspondence between the AN model with negative gamma distribution and the BN model can be extended to arbitrary $k$ by 
setting the shape parameter to $s=1 / k$. In particular,

$$
\lambda_{k}^{\mathrm{AN}}=\lambda_{k}^{\mathrm{BN}}=\left(\frac{1}{k+1}\right)^{1 / k} \text { for } p_{f}(x)=g_{1 / k}(-x) .
$$

Moreover, a variational analysis around the negative gamma distribution with shape parameter $1 / k$, viewed as a point in the probability distribution space, proves that $\lambda_{k}^{\mathrm{AN}}$ is not only maximized along the $s$-axis but also extremized in the whole space of distributions with support limited to the negative real axis. This observation corroborates the conjecture [78 that the BN model growth rate $\lambda_{k}^{\mathrm{BN}}$ is an upper bound on $\lambda_{k}$ among all possible NK structures.

Next we discuss how our method can be generalized to larger values of $k$. In order to avoid notational clutter, it is best to consider $k=3$. In this particular case, we can construct a transfer matrix having state space $\mathbb{R}^{2}$ :

$$
\begin{aligned}
K_{w}\left(x_{1}, x_{2} ; y_{1}, y_{2}\right)= & p_{f}\left(x_{1}\right)^{w} p_{f}\left(x_{2}\right)^{w} \tilde{F}^{(3)}\left(x_{1}+x_{2}+y_{1}\right) \\
& \times \tilde{F}^{(3)}\left(x_{2}+y_{1}+y_{2}\right) p_{f}\left(y_{1}\right)^{1-w} p_{f}\left(y_{2}\right)^{1-w},
\end{aligned}
$$

for $0 \leq w \leq 1$. By expanding the state space to $\mathbb{R}^{k-1}$, a similar construction can be made for higher values of $k$. Furthermore, once the kernel is constructed, all the procedures described for $k=2$ may be applied for arbitrary $k$ as long as the kernel is separable. However, we found that the direct application of this approach for the gamma distribution becomes quickly unmanageable, because the dimension of the transfer matrix increases combinatorially fast. The only result known from the literature for $k>2$ is the value $\lambda_{3}^{\mathrm{AN}} \simeq 0.61140$ for the exponential distribution [15].

Despite this limitation, one may still perform an asymptotic analysis for $\lambda_{k}^{\mathrm{AN}}$. In particular, for the Gaussian distribution, it is rigorously known that 43

$$
\ln \lambda_{k}^{\mathrm{AN}}=-\frac{1}{k}\left(\ln k+R_{L, k}\right)
$$

where $-c \sqrt{\ln k} \leq R_{L, k} \leq c \ln \ln k$ for some $c>0$. For arbitrary distributions the same authors establish the inequality

$$
-\frac{3}{k}(\ln k+o(1)) \leq \ln \lambda_{k}^{\mathrm{AN}} \leq-\frac{1}{k}(\ln k+o(1))
$$

up to discreteness effects in $L / k$. They conjecture that the coefficient 3 in the lower bound can be replaced by 1 , and corroborate this claim by improved bounds for two classes of heavy-tailed base distributions. Taken together with the identity (57), these results lend strong support to the idea that the AN and BN models belong to the same universality class of NK structures, in the sense that $\ln \lambda_{k}^{\mathrm{AN}, \mathrm{BN}}=-\frac{\ln k}{k}$ to leading order in $k$ and $\pi_{\max }^{\mathrm{AN}, \mathrm{BN}} \sim L^{-1 / \alpha}$ in the joint limit. 


\subsubsection{Random neighborhood}

Although the random NK structure has been one of the most commonly studied neighborhood structures in the literature, little is known about the analytic behavior of $\lambda_{k}^{\mathrm{RN}}$; in fact the existence of a well-defined exponential growth rate for \# max has been rigorously established only for the AN model 14. In contrast to the BN or AN interaction structures which are defined in a deterministic manner, the RN model is marginally structured, in the sense that the neighborhood sets are realizations drawn from a random ensemble. Thus, it is of interest to ask how this marginal structure influences the behavior of $\lambda_{k}^{\mathrm{RN}}$, now that we have seen that the maximally unstructured MF model belongs to a different universality class than the $\mathrm{AN}$ and $\mathrm{BN}$ models. In order to answer this question, we choose to study the regular random NK structure $(\mathrm{rRN})$ as defined in Sec. 2.7. The regular structure is chosen because it turns out to be analytically tractable. However, we claim that whether we assume regularity or uniformity on the NK structures should not matter for sufficiently large $k$, since the fluctuations in the locus degrees or the size of NK blocks decay as $k^{-1 / 2}$. We later numerically confirm that this is indeed true.

To proceed, let us first examine Eq. (24). In contrast to the previously studied models with deterministic NK structures, the elements of the incidence matrix $b_{l, r}$ in the RN models may be considered as binary random variables constrained by the conditions i) $b_{l, l}=1$ and ii) $\sum_{r} b_{l, r}=k$ for all $l[12$. The second condition ensures that the underlying NK structures are regular while the first condition represents the self-link condition imposed on classical NK structures. In our analysis, we found that the first condition does not play any significant role while introducing unnecessary complication. Because the variable influenced by this condition is only one out of $k$ variables for each locus, the effect due to this condition should be at most $O\left(k^{-1}\right)$. Thus, as long as we focus on the leading asymptotic behavior, the condition i) can be dropped in the following analysis.

The average over different realizations of the rRN NK structure can now be emulated by promoting the $b_{l, r}$ to Bernoulli random variables. These variables are assumed to be i.i.d with the Bernoulli success probability $p / L$ where $p$ is an arbitrary fixed constant in the limit $L \rightarrow \infty$. Then, the average of a random quantity $Q$ over the rRN NK structure is given by

$$
\langle Q\rangle \equiv \mathcal{N}^{-1}\left\langle Q \prod_{l} \delta_{\sum_{r} b_{l, r}, k}\right\rangle_{\left\{b_{l, r}\right\}},
$$

where the angular bracket with subscript $\langle\cdots\rangle_{\left\{b_{l, r}\right\}}$ indicates the average over the Bernoulli variables $\left\{b_{l, r}\right\}$, and we have introduced a normalization constant

$$
\mathcal{N}=\left\langle\prod_{l} \delta_{\sum_{r} b_{l, r}, k}\right\rangle_{\left\{b_{l, r}\right\}} .
$$

Our goal is to evaluate Eq. 61 for the quantity of interest, i.e., $Q=\pi_{\max }^{\mathrm{rRN}}$. 
The normalization constant $\mathcal{N}$ is relatively simple to calculate. Since the $\left\{b_{l, r}\right\}$ are independent Bernoulli variables, the total weight is given by the binomial distribution,

$$
\mathcal{N}=\left[\left(\begin{array}{l}
L \\
k
\end{array}\right)\left(1-\frac{p}{L}\right)^{L-k}\left(\frac{p}{L}\right)^{k}\right]^{L} \simeq\left[\frac{e^{-p} p^{k}}{k !}+O(1 / L)\right]^{L}
$$

For the average of $Q$, it is convenient to use an integral representation for the Kronecker delta symbol,

$$
\delta_{x, n}=\frac{1}{2 \pi} \int_{0}^{2 \pi} e^{i(x-n) t} d t
$$

By combining this equation with Eq. 25, we may set up our starting equation for $\left\langle\pi_{\max }^{\mathrm{rRN}}\right\rangle$ as

$$
\begin{aligned}
& \left\langle\pi_{\max }^{\mathrm{rRN}} \prod_{l} \delta_{\sum_{r} b_{l, r}, k}\right\rangle_{\left\{b_{l, r}\right\}}=\int \mathcal{D} \mathbf{t} \mathcal{D} \mathbf{y} \mathcal{P}(\mathbf{y}) \frac{\mathcal{D} \mathbf{u} \mathcal{D} \mathbf{q}}{(2 \pi)^{L}} e^{-i \mathbf{u} \cdot \mathbf{q}} \Theta(\mathbf{u}>0) \\
& \times \Theta(0<\mathbf{t}<2 \pi)\left\langle e^{\sum_{l} i t_{l}\left(\sum_{r} b_{l, r}-k\right)} \prod_{r, l}\left[\phi_{f}\left(-q_{l}\right) e^{i y_{r} q_{l}}\right]^{b_{l, r}}\right\rangle_{\left\{b_{l, r}\right\}} .
\end{aligned}
$$

Here, we introduced another theta function $\Theta(0<\mathbf{t}<2 \pi)$ enforcing the condition $t_{l} \in(0,2 \pi)$ for all $l$. After averaging $\left\{b_{l, r}\right\}$ and neglecting terms of $O(1)$ in the exponential, one finds

$$
\begin{aligned}
& \left\langle e^{\sum_{l} i t_{l}\left(\sum_{r} b_{l, r}-k\right)} \prod_{r, l}\left[\phi_{f}\left(-q_{l}\right) e^{i y_{r} q_{l}}\right]^{b_{l, r}}\right\rangle_{\left\{b_{l, r}\right\}} \\
& =\left(\prod_{l} e^{-i t_{l} k}\right) \exp \left[-L p+\frac{p}{L} \sum_{l, r} \phi_{f}\left(-q_{l}\right) e^{i y_{r} q_{l}+i t_{l}}\right],
\end{aligned}
$$

where we used the fact that $\left\langle e^{b_{l, r} x}\right\rangle_{\left\{b_{l, r}\right\}}=1-\frac{p}{L}\left(1-e^{x}\right)=e^{-\frac{p}{L}\left(1-e^{x}\right)+O\left(L^{-2}\right)}$. By defining a quantity $\psi\left(q_{l}\right)=\frac{1}{L} \sum_{r} e^{i q_{l} y_{r}}$, the last term in the square bracket is succinctly written as

$$
\frac{p}{L} \sum_{l, r} \phi_{f}\left(-q_{l}\right) e^{i y_{r} q_{l}+i t_{l}}=p \sum_{l} e^{i t_{l}} \phi_{f}\left(-q_{l}\right) \psi\left(q_{l}\right)
$$

After taking a short glance at the definition of $\psi\left(q_{l}\right)$, it is tempting to claim that this is simply the characteristic function of the base density function $p_{f}(y)$, because $\mathbf{y}=\left\{y_{r}\right\}$ is drawn from the probability measure $\mathcal{D} \mathbf{y} \mathcal{P}(\mathbf{y})=$ $\prod_{r} d y_{r} p_{f}\left(y_{r}\right)$. As long as $\mathbf{y}$ is a typical realization, this claim must be true. However, we cannot make this assumption, because the values of the $y_{r}$ conditioned on being a local maximum may not be typical. Instead, we will 
call $\psi\left(q_{l}\right)$ the sample characteristic function realized by $\mathbf{y}$. Due to the structural similarity, this allows a (cumulant) expansion of the form

$$
\ln \psi\left(q_{l}\right)=i q_{l} Y_{1}-\frac{q_{l}^{2}}{2}\left(Y_{2}-Y_{1}^{2}\right)+O\left(q_{l}^{3}\right),
$$

where $Y_{m}=\frac{1}{L} \sum_{r} y_{r}^{m}$, the $m$-th sample moment. However, one should keep in mind that the $Y_{m}$ are random variables which depend on the random vector $\mathbf{y}$.

Now we are ready to evaluate the integrals over $t_{l}$. After applying the identity

$$
\frac{1}{2 \pi} \int_{0}^{2 \pi} e^{-i Q t+x e^{i t}} d t=\frac{x^{Q}}{Q !}
$$

to each of the integrals with respect to $t_{l}$, we may factor out the equation as

$$
\begin{aligned}
& \left\langle\pi_{\max }^{\mathrm{rRN}} \prod_{l} \delta_{\sum_{r} b_{l, r}, k}\right\rangle_{\left\{b_{l, r}\right\}} \\
& =\int \mathcal{D} \mathbf{y} \mathcal{P}(\mathbf{y})\left[\int_{0}^{\infty} \frac{d u}{2 \pi} \int_{-\infty}^{\infty} d q e^{-i u q-p} \frac{\left[p \psi(q) \phi_{f}(-q)\right]^{k}}{k !}\right]^{L} .
\end{aligned}
$$

Finally, dividing by the normalization constant Eq. 63 yields

$$
\left\langle\pi_{\max }^{\mathrm{rRN}}\right\rangle=\int \mathcal{D} \mathbf{y} \mathcal{P}(\mathbf{y})\left[\int_{0}^{\infty} \frac{d u}{2 \pi} \int_{-\infty}^{\infty} d q e^{-i u q}\left[\psi(q) \phi_{f}(-q)\right]^{k}\right]^{L} .
$$

As expected from the fact that $p$ was introduced as an arbitrary parameter, the dependence on $p$ completely vanishes in the final equation.

Next the $u$ and $q$ integrals in the square bracket may be evaluated by means of the steepest descent method assuming $k$ is sufficiently large. Reflecting the fact that $u$ can be arbitrarily large, $u$ is rescaled to $k u$ to have the same order in $k$ in the exponential. Moreover, since the result of the integral should be real-valued, it is convenient to perform a complex rotation $q \rightarrow i q$. Rewriting Eq. (71) and denoting the integral in the square bracket by $I$, we have

$$
I=i k \int_{0}^{\infty} \frac{d u}{2 \pi} \int_{-\infty i}^{\infty i} d q \exp [k(u q-f(q))]
$$

where

$$
f(q)=-\ln \left[\psi(i q) \phi_{f}(-i q)\right] .
$$

Then, the steepest contour is determined such that it passes through the saddle point satisfying the equation $u-f^{\prime}\left(q_{c}\right)=0$. Along this contour, one finds

$$
I=i k \int_{0}^{\infty} \frac{d u}{\sqrt{2 \pi}} \exp \left[k\left(u q_{c}-f\left(q_{c}\right)\right)\right] \times \frac{1}{\sqrt{k f^{\prime \prime}\left(q_{c}\right)}} .
$$


Subsequently, yet another saddle point approximation to the $u$ integral gives

$$
I=\sqrt{\frac{-k}{f^{\prime \prime}\left(q_{c}\right)}} \int_{0}^{\infty} \frac{d u}{\sqrt{2 \pi}} \exp \left[k q_{c}^{\prime}\left(u_{c}\right)\left(u-u_{c}\right)^{2}\right]=\frac{\operatorname{erf}\left(\frac{\sqrt{k} f^{\prime}(0)}{\sqrt{-2 f^{\prime \prime}(0)}}\right)+1}{2}
$$

where $u_{c}$ is defined by the relation $q_{c}\left(u_{c}\right)=0$ (or equivalently $u_{c}=f^{\prime}(0)$ ) and we used the reciprocal relation $q_{c}^{\prime}(u) \frac{1}{f^{\prime \prime}\left(q_{c}(u)\right)}=1$ well known in the context of the Legendre transformation. Surprisingly, if we are only interested in the leading behavior, this integral only depends on the two quantities $f^{\prime}(0)$ and $f^{\prime \prime}(0)$. Using the cumulant expansion Eq. (68), we may rewrite $I$ in terms of the first two moments as

$$
I\left(Y_{1}, Y_{2}\right)=\frac{1}{2}\left(\operatorname{erf}\left(\left(Y_{1}-m_{1}\right) \sqrt{\frac{k}{2\left(Y_{2}-Y_{1}^{2}+m_{2}-m_{1}^{2}\right)}}\right)+1\right)
$$

up corrections of the order of $k^{-1}$, where $m_{q}$ denotes the $q$-th moment of the base distribution $p_{f}$. We emphasize that $I$ depends only on $Y_{1}$ and $Y_{2}$ by explicitly specifying them as the arguments of $I$. Exploiting the fact that $\pi_{\max }$ is not affected by translation and scaling, we may take $m_{1}=0$ and $m_{2}=1$ without loss of generality. Hence, we have

$$
I\left(Y_{1}, Y_{2}\right)=\frac{1}{2}\left(\operatorname{erf}\left(Y_{1} \sqrt{\frac{k}{2\left(Y_{2}-Y_{1}^{2}+1\right)}}\right)+1\right)\left(1+O\left(k^{-1}\right)\right) .
$$

Since $I$ depends only on $Y_{1}$ and $Y_{2}$, the remaining task for the integral over $\mathbf{y}$ is to calculate the joint probability

$$
J\left(Y_{1}, Y_{2}\right) \equiv L^{2} \int \mathcal{D} \mathbf{y} \mathcal{P}(\mathbf{y}) \delta\left(L Y_{1}-\sum_{r} y_{r}\right) \delta\left(L Y_{2}-\sum_{r} y_{r}^{2}\right) .
$$

As $Y_{1}$ and $Y_{2}$ are sums of a large number of random variables, the large deviation principle implies that the joint probability should be of the form $J\left(Y_{1}, Y_{2}\right) \sim e^{L \mathcal{J}\left(Y_{1}, Y_{2}\right)}$, where $\mathcal{J}\left(Y_{1}, Y_{2}\right)$ is the corresponding rate function. Once the joint probability is obtained for the given base distribution, we are finally ready to evaluate $\pi_{\max }^{\mathrm{rRN}}$ by means of the saddle point method,

$$
\begin{aligned}
\pi_{\max }^{\mathrm{rRN}} & =\int d Y_{1} d Y_{2} J\left(Y_{1}, Y_{2}\right) I\left(Y_{1}, Y_{2}\right)^{L} \\
& \sim \exp \left[L \mathcal{F}^{\mathrm{rRN}}\left(Y_{1}^{*}, Y_{2}^{*}\right)\right]
\end{aligned}
$$

where the starred variables $\left(Y_{1}^{*}, Y_{2}^{*}\right)$ represent the solution of the extremum conditions on the action

$$
\mathcal{F}^{\mathrm{rRN}}\left(Y_{1}, Y_{2}\right)=\mathcal{J}\left(Y_{1}, Y_{2}\right)+\mathcal{I}\left(Y_{1}, Y_{2}\right) \text { with } \mathcal{I}\left(Y_{1}, Y_{2}\right) \equiv \ln I\left(Y_{1}, Y_{2}\right) .
$$

As an example, let us suppose that our base distribution is a standard normal distribution. This particular choice makes the calculation of the joint 
probability relatively easy. Using the integral representation of the delta function, the joint distribution Eq. (78) may be written as

$$
\begin{aligned}
J\left(Y_{1}, Y_{2}\right) & =\frac{L^{2}}{(2 \pi)^{2}} \int d Z_{1} d Z_{2} \int \prod_{r} \frac{d y_{r}}{\sqrt{2 \pi}} e^{-L \frac{Y_{2}}{2}} \\
& \times \exp \left(i L Y_{1} Z_{1}+i L Y_{2} Z_{2}-i Z_{1}\left[\sum_{r} y_{r}\right]-i Z_{2}\left[\sum_{r} y_{r}^{2}\right]\right) \\
& =\frac{L^{2}}{(2 \pi)^{2}} \int d Z_{1} d Z_{2} e^{L\left[-\frac{Y_{2}}{2}+i Y_{1} Z_{1}+i Y_{2} Z_{2}+\frac{i Z_{1}^{2}}{4 Z_{2}}-\frac{1}{2} \ln \left(2 i Z_{2}\right)\right]} \\
& \sim \exp \left[\frac{L}{2}\left\{1-Y_{2}+\ln \left(Y_{2}-Y_{1}^{2}\right)\right\}\right],
\end{aligned}
$$

where we have used the fact that the solution of the extremum conditions for $Z_{1}$ and $Z_{2}$ is given by $Z_{1}=-\frac{i Y_{1}}{Y_{1}^{2}-Y_{2}}, Z_{2}=\frac{i}{2\left(Y_{1}^{2}-Y_{2}\right)}$. Once $J\left(Y_{1}, Y_{2}\right)$ is obtained, $\lambda_{k}^{\mathrm{rRN}}$ is readily calculated by combining Eq. 81 with Eq. 79 .

Now, we are ready to uncover the universal behavior hidden in Eq. (79). To describe it clearly, let us consider the limit $k \rightarrow \infty$ first. Note that the dependence on $k$ only appears in $\mathcal{I}\left(Y_{1}, Y_{2}\right)$. Examining the behavior of the error function shows that this limit effectively makes $\mathcal{I}\left(Y_{1}, Y_{2}\right)$ vanish. Furthermore, in the absence of the term $\mathcal{I}\left(Y_{1}, Y_{2}\right)$ in Eq. 80 that prefers certain non-typical realizations of $\mathbf{y}$, it is clear that the saddle point $\left(Y_{1}^{*}, Y_{2}^{*}\right)$ is given by the typical realizations, namely $Y_{1}^{*}=0$ and $Y_{2}^{*}=1$. Hence, if we introduce the variables $\epsilon_{q}=Y_{q}-m_{q}$ for $q \in\{1,2\}$, they are expected to vanish for sufficiently large $k$, and this allows us to perform a series expansion with respect to these variables. Using the general property of large deviation functions that the lowest order terms are given by a covariance matrix of $Y_{1}$ and $Y_{2}$ [86, we find that

$$
\mathcal{J}\left(\epsilon_{1}, \epsilon_{2}\right)=\sum_{p, q \in\{1,2\}}-\frac{1}{2} \epsilon_{p} \Sigma_{p q}^{-1} \epsilon_{q}+O\left(\text { cubic in } \epsilon_{q}\right),
$$

where $\Sigma$ is the covariance matrix among $\epsilon_{1}$ and $\epsilon_{2}$. Specifically, the values are given by $\Sigma_{11}=L \mathbb{E}\left[\epsilon_{1}^{2}\right]=1, \Sigma_{12}=\Sigma_{21}=L \mathbb{E}\left[\epsilon_{2} \epsilon_{1}\right]=m_{3}$ and $\Sigma_{22}=$ $L \mathbb{E}\left[\epsilon_{2}^{2}\right]=m_{4}-m_{2}^{2}=m_{4}-1$.

On the other hand, the expansion for $\mathcal{I}\left(\epsilon_{1}, \epsilon_{2}\right)$ should be performed with caution due to the fact that $\sqrt{k} \epsilon_{1}$ cannot be assumed to be a small variable. Instead, the order of $\epsilon_{1}$ will be determined through this combined variable when $k$ is taken to $\infty$. Namely, the expansion takes the form

$$
\mathcal{I}\left(\epsilon_{1}, \epsilon_{2}\right)=g\left(\sqrt{k} \epsilon_{1}\right)+g^{\prime}\left(\sqrt{k} \epsilon_{1}\right) \frac{\epsilon_{1} \sqrt{k}}{8} \epsilon_{2}+O\left(\text { quadratic in } \epsilon_{q}\right)
$$

with $g\left(\sqrt{k} \epsilon_{1}\right)=\ln \left[\frac{1}{2} \operatorname{erf}\left(\epsilon_{1} \sqrt{\frac{k}{4}}\right)+\frac{1}{2}\right]$. Note that to lowest order, only $\epsilon_{1}$ appears. Thus, as far as this leading order is concerned, the extremum condition for $\epsilon_{2}$ is readily solved by $\epsilon_{2}=-\frac{\Sigma_{21}^{-1}}{\Sigma_{22}^{-1}}$, which then leads us to write the 

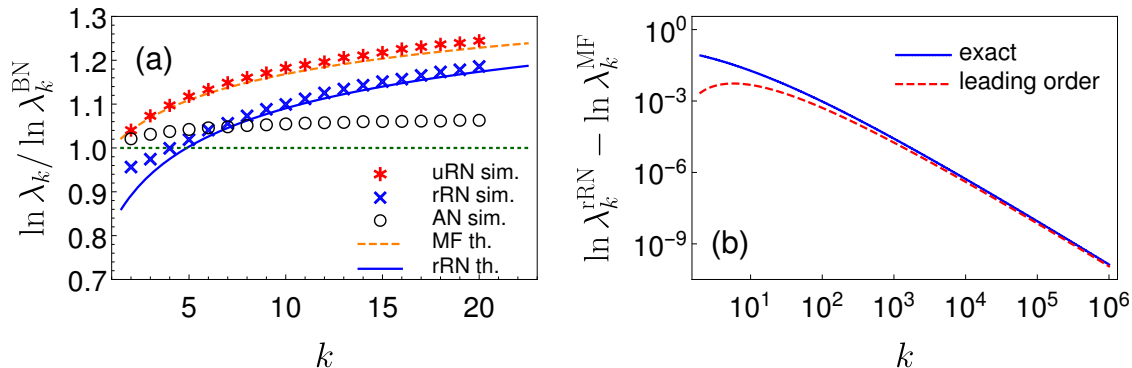

Fig. 5 (a) Plots of $\ln \lambda_{k} / \ln \lambda_{k}^{\mathrm{BN}}$ for various NK structures. The standard normal distribution is chosen as the base distribution for the simulations. The symbols denote the simulation data for each NK structure: stars for uRN model, crosses for rRN model and open circles for the AN model. In addition, analytical results for MF model (dashed) Eq. (34), rRN model (solid) Eq. (79) and BN model Eq. (26) (dotted) are drawn, respectively. Because the exponentral growth rate is rescaled by $\lambda_{k}^{\mathrm{BN}}$, the curve for $\mathrm{BN}$ is constant at 1 . The results for the two types of RN model converge to the asymptotic behavior of the MF model, while the curve for the AN model is expected to converge to one. (b) Plot of higher order corrections in the rRN model. The exact value is calculated from Eq. (79) while the approximate value is obtained from Eq. (84). This shows that the leading correction behaves as $\frac{(\ln k)^{2}}{16 k^{2}}$.

action as a one-dimensional function

$$
\mathcal{F}^{\mathrm{MF}}\left(\epsilon_{1}\right)=-\frac{1}{2} \epsilon_{1}^{2}+\ln \left[\frac{1}{2} \operatorname{erf}\left(\epsilon_{1} \sqrt{\frac{k}{4}}\right)+\frac{1}{2}\right],
$$

regardless of the specific form of the covariance matrix $\Sigma$. Surprisingly, if we identify $\epsilon_{1}$ with $y$, this is exactly the MF action Eq. (35) to leading order in $k$. Thus, for sufficiently large $k$, the solution of Eq. (79) should converge to the MF solution. This is confirmed by the simulation results shown in Fig. 5 (a).

Next we turn to the corrections to the leading behavior. Since these depend on the next-order terms of $\mathcal{J}\left(\epsilon_{1}, \epsilon_{2}\right)$ which contain higher order correlations between $\epsilon_{1}$ and $\epsilon_{2}$, it is evident that this behavior is less universal than Eq. (84). Thus, in general not much can be said except the overall order of the corrections, which is at most $O\left(\left(\epsilon_{1}^{*}\right)^{3}\right)$ (here the argument maximizing Eq. (84) is denoted by $\left.\epsilon_{1}^{*}\right)$. Nevertheless, the next-order correction can be computed on a case by case basis once a distribution is given. For the Gaussian distribution, a simple analysis shows that the next order correction of the saddle point equation gives $\epsilon_{2}^{*}=0 \epsilon_{1}^{*}+\frac{1}{2}\left(\epsilon_{1}^{*}\right)^{2}+O\left(\left(\epsilon_{1}^{*}\right)^{3}\right)$ and thus we arrive at

$$
\mathcal{F}^{\mathrm{rRN}}\left(\epsilon_{1}^{*}, \epsilon_{2}^{*}\right)=\mathcal{F}^{\mathrm{MF}}\left(\epsilon_{1}^{*}\right)+\frac{1}{16}\left(\epsilon_{1}^{*}\right)^{4}+O\left(\left(\epsilon_{1}^{*}\right)^{5}\right) .
$$

Using the asymptotic expansion Eq. 38 , we found that this correction is of the order of $\frac{(\ln k)^{2}}{k^{2}}$ (See Fig. $5(\mathrm{~b})$ ). 


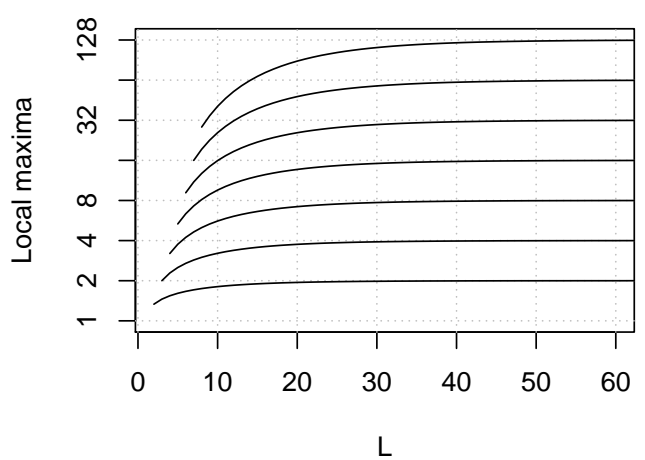

Fig. 6 Mean number of local maxima for the SN structure versus number of loci $L$. From bottom to top the lines represent the cases of $k=2$ to 8 . For large $L$ the number of maxima converges to $2^{k-1}$. Gaussian base fitness was used.

Now that we have established the universal behavior of $\lambda_{k}$ for the case of the rRN model, it would be interesting to see if it applies also to other versions of the RN model, e.g., the uniform ( $\mathrm{uRN}$ ) model. For the sake of comparison we have performed simulations of this model with a standard normal distribution as the base distribution. This choice is made since it allows for an efficient numerical computation, which was first suggested in [6] (see Appendix D for the details of the algorithm). Also, in order to test the effect of the self-link condition $b_{l, l}=1$ which has been ignored in the analytical calculation, the simulations were performed in the presence or the absence of this condition. Fortunately, we found no significant difference between the results on the scale of Fig. 5 and thus each NK structure is represented by a single curve without specifying whether the condition was implemented or not.

The simulation results turn out to be quite surprising in the sense that $\lambda_{k}^{\mathrm{uRN}}$ is extremely close to $\lambda_{k}^{\mathrm{MF}}$ for all the parameter ranges we checked. This supports our claim that a wide class of RN models is asymptotically MF-like as long as $k$ is sufficiently large.

Finally, we emphasize that the seemingly constant gaps shown in Fig. 5 between the simulation data and the theoretical curves for the RN models are artifacts that originate from the normalization by $\ln \lambda_{k}^{\mathrm{BN}}$. In order to justify this statement, recall that our solution Eq. (77) is correct up to the order of $O\left(k^{-1}\right)$. Since the results are rescaled by $\lambda_{k}^{\mathrm{BN}} \sim \frac{\ln k}{k}$, the gaps decay only as $(\ln k)^{-1}$, which effectively remains constant over the range of $k$ covered in the simulations.

\subsubsection{Star neighborhood}

As our last example of NK structures we consider the star neighborhood $(\mathrm{SN})$ introduced in Sec. 2.7. In contrast to all other structures discussed so far, the number of local maxima in the SN model remains finite for $L \rightarrow \infty$ and hence formally $\lambda_{k}^{\mathrm{SN}}=1 / 2$ (see Fig. 6).

The analysis of the SN model can largely be based on combinatorial arguments. Suppose the $k-1$ center loci are fixed in a certain configuration, and let us first determine the number of local maxima with respect to the remaining $L-k+1$ ray loci under this background. Since none of the ray loci 
appear together in any NK edge, a mutation on one of them cannot affect the sign of the mutational effect on another, i.e. they are pairwise completely non-epistatic. Thus each ray locus can be mutated into its state contributing higher fitness and this state is the unique (global) fitness maximum in the subspace of ray loci for the given background of center loci. Since this is true for every allele combination of center loci, there can be at most $2^{k-1}$ local maxima on the star neighborhood. This by itself already proves that $\lambda_{k}^{\mathrm{SN}}=1 / 2$.

For the scaling limit $L \rightarrow \infty$ at constant $k$, we can find bounds on the probability that the candidates for local maxima identified above are actually realized. Because a mutation on a center locus affects all NK edges, the ray locus sub landscapes for each background of center loci are statistically independent. Therefore, starting from the local maximum candidate constructed for one allele combination of the center loci and applying a mutation to one of the center loci, the new fitness value $F^{\prime}$ is a sum of $L$ i.i.d. random variables drawn from $p_{f}$. This is to be compared to the fitness value $F^{\text {cand }}$ of the candidate configuration, which was obtained by maximizing each of the $L-k+1$ ray locus contributions between the two possible states of that locus. Thus $F^{\text {cand }}$ is the sum of maxima of $L-k$ pairs of random variables drawn from $p_{f}$, plus one maximum of a pair of random variables drawn from the $k$-fold convolution of $p_{f}$; the last contribution originates from the special ray locus which is contained in all the blocks associated with the center loci.

Except for the deterministic distribution, the expected value of the maximum of two independent draws from a probability distribution is always greater than the mean of the distribution itself, and therefore the mean of $F^{\text {cand }}-F^{\prime}$ grows linearly in $L$. At the same time the variance, as long as it exists, also grows linearly in $L$. Thus by Chebyshev's inequality the probability for the mutation to lead us to discard the candidate local maximum is decreasing as $\frac{1}{L}$. As the number of possible mutations of the center loci is also independent of $L$, it follows that the probability of each of the local maximum candidates not to be an actual local maximum is decreasing as $\frac{1}{L}$. Therefore at constant $k, \mathbb{E}\left[\#_{\max }^{\mathrm{SN}}\right]=2^{k-1}\left(1-\mathcal{O}\left(\frac{1}{L}\right)\right)$. Because we also know that $2^{k-1}$ is a strict upper bound, it follows that $\lim _{L \rightarrow \infty} \mathbb{P}\left[\#_{\max }^{\mathrm{SN}} \neq 2^{k-1}\right]=0$.

\subsection{Summary}

In this section, we have investigated the expected value of the number of local fitness maxima for various NK structures. By developing a new analytic framework that allows us to treat different structures in a unified manner, we have discovered that the exponential growth rate of this quantity behaves asymptotically as

$$
\ln \lambda_{k} \sim-\beta \frac{\ln k}{k}
$$

in the large $k$ limit, with the coefficient $\beta$ taking the values $\beta=1$ for the $\mathrm{AN}$ and BN models and $\beta=2$ for the MF and RN models. Similarly in the joint limit $k, L \rightarrow \infty$ at fixed $\alpha=k / L$, the probability $\pi_{\max }$ that a random 
genotype is a local maximum decays algebraically as

$$
\pi_{\max } \sim L^{-\mu}
$$

with $\mu=1 / \alpha$ for the $\mathrm{AN}$ and BN models, and $\mu=2 / \alpha-1$ for the MF and RN models. The latter result has so far been established only for the MF model, where it is modified by a logarithmic correction (Appendix A). Although the change from $\beta=1$ to $\beta=2$ in Eq. 86 may not seem very dramatic, it is important to note that the corresponding numbers of fitness maxima \# $\#_{\max }$ differ by a factor of $k^{L / k}$, which can be large already for moderate values of $k$ and $L$.

Because the AN and BN models can be considered to be more structured in a certain sense, these results suggest that the fitness landscape is more rugged when the NK structure is more organized. A similar conclusion was reached in 66 and 54, where it was found that the number of maxima correlates negatively with the rank (16) of the NK structure. Note, however, that the SN structure does not conform to this pattern, as its rank is relatively low (between the BK and AN models, see Table 1) whereas the number of maxima remains finite for $L \rightarrow \infty$.

As a next question, one might ask if other values of $\beta$ can be found or even further if other types of functional behavior can be realized for certain choices of NK structures. Given the large variety of NK structures that is allowed by the definition of the model, the answers to both questions turn out be affirmative. To answer the first question, let us consider a somewhat contrived example. First, let us split the genotype sequence into two pieces of size $L \rho$ and $L(1-\rho)$, respectively. Furthermore, suppose that there is one NK block associated to each locus. Next, let us assume that the NK blocks associated to the loci belonging to the first piece are constructed as if it were a BN model of size $L \rho$. For the second piece, the NK blocks are created as in an RN model. Since there is no overlap between these two pieces by construction, the total number of local maxima is simply the product of those in each subsystem. From this, one may conclude that the asymptotic behavior of the exponential growth factor should be

$$
\ln \lambda_{k}=-[\rho+2(1-\rho)] \frac{\ln k}{k} .
$$

Thus, depending on the parameter $\rho$, the value of $\beta$ varies continuously from 2 to 1 . However, this model does not allow for values that are outside of the range $1 \leq \beta \leq 2$. In this sense, the value of $\beta$ is a measure of the amount of structure in the NK model.

In Appendix C we prove that $\beta \in[1,2]$ for all uniform and regular structures if Gaussian fitness is assumed. We expect this to hold for all sufficiently regular structures, although the proof is likely to be somewhat more complicated. Whether $\beta$ can take on other values if the base fitness distribution is varied is open. Preliminary unpublished results for an extremely heavy-tailed distribution suggested in [43] seem to indicate that the relation Eq. (86) may not even hold for certain uniform regular structures. Nonetheless we expect at least distributions with finite moments to result in behavior equivalent to 
the Gaussian case, since for large $k$ fitness differences effectively converge to a jointly normal distribution following a kind of central limit theorem.

With regard to the second question, the example of the star neighborhood in Sec. 3.2.5 shows that the exponential growth of the number of maxima with $L$ is not a general feature even among the classical NK structures. We attribute this inherently different behavior to the extreme non-regularity of the SN structure, where certain loci appear a macroscopic number of times. Extending our analysis to other such non-regular structures might be an interesting future direction to further clarify the behavior of $\lambda_{k}$.

\section{Accessible pathways}

\subsection{Definitions}

There are many paths between far away genotypes. However, some paths may be harder to take for a population, with some quasi impossible to take. A path is called accessible if it increases fitness in each step 93, 95. This in particular implies that accessible paths are never circular and that no genotypes can be visited twice on an accessible path.

We say a path from $\sigma$ to $\theta$ is direct if $d_{h}(\sigma, \theta)$ is the number of steps taken, i.e. if the path has minimal length, and indirect otherwise 101. The number of allelic states $A$ is largely irrelevant for the analysis of direct paths. In contrast, indirect paths become more complex for $A>2$ because of the possibility of distance-neutral mutations that neither increase nor decrease the distance to the target 102 . Here we mostly restrict our analysis to the biallelic case, where the genotype spaces are hybercubes. Our results for the NK-model presented in Sect. 4.4 can however be straightforwardly generalized to multiple alleles.

Direct paths on the hypercube mutate each locus at most once, i.e. there are no backwards mutations or mutational reversions [13. On the hypercube there exist exactly $\left(d_{h}(\sigma, \theta)\right)$ ! direct paths between any two genotypes, in particular there are $L$ ! direct paths between a genotype and its antipode. The total number of (simple) paths including indirect paths is much larger, see 5 . In the following we denote the total number of accessible paths by $\#_{\mathrm{p}}[\sigma \rightarrow \theta]$ and the number of direct accessible paths by $\#_{\mathrm{dp}}[\sigma \rightarrow \theta]$. If these numbers are non-zero we say that $\theta$ is (direct) accessible from $\sigma$.

Of particular interest are paths from a genotype $\sigma$ to its antipodal $\Delta_{\mathcal{L}} \sigma$ as an approximate worst-case scenario. Many genotypes are not accessible from their antipodal purely because their fitness is low compared to their neighbors. As these cases are not very interesting, one may focus on high-fitness final genotypes. Here looking at local maxima and in particular the global maximum $\Omega$ as destination seems natural 9.19 . We use the short-hand notation $\#_{\mathrm{dp} \Omega}=\#_{\mathrm{dp}}\left[\Delta_{\mathcal{L}} \Omega \rightarrow \Omega\right]$ and $\#_{\mathrm{p} \Omega}=\#_{\mathrm{p}}\left[\Delta_{\mathcal{L}} \Omega \rightarrow \Omega\right]$ respectively for direct and arbitrary paths to the global maximum from its antipodal. The number of direct and indirect accessible paths to the global maximum has been studied for different fitness landscape models. A major question of interest is the probability of existence of such paths for a large number 
of loci. This problem is non-trivial. On the one hand the number of possible paths between antipodal genotypes increases factorially (direct paths) or faster (indirect paths) with the number of loci. On the other hand the number of fitness values needed to be found in monotonic order for a path to be accessible increases as well. This bears similarity to certain percolation problems. Therefore also the term accessibility percolation has been used to describe the probability of existence of paths to the global maximum from its antipodal 53 .

Practically it is for some models, such as the NK model, difficult to condition on the global maximum. Therefore it may be useful to consider a class of accessible paths larger than those discussed in the previous paragraph to describe a percolation property of the fitness landscape. We call a landscape (direct) traversable if there exists a pair of genotypes at maximal distance $L$ with an accessible (direct) path between them. This definition is more similar to traditional percolation problems, as no additional conditioning on the global maximum is required.

\subsection{House-of-Cards model}

Accessible paths to the global maximum from the antipodal point have been studied in detail in the limit of $L \rightarrow \infty$. A simple combinatorial argument shows that $\mathbb{E}\left[\#_{\mathrm{dp} \Omega}\right]=1$ in the HoC model [19]. For this notice that any given direct path to the global maximum is accessible if all $L$ involved genotypes, excluding the global maximum itself, are ordered in ascending order. Because all these values are i.i.d. this probability is $\frac{1}{L !}$. As there are $L$ ! such paths, the claim follows.

The distribution of $\#_{\mathrm{dp} \Omega}$ however becomes highly skewed for larger $L$, which implies that the mean is not informative of the typical behavior. Using the second moment method Hegarty and Martinsson showed that [29]

$$
\mathbb{P}\left[\#_{\mathrm{dp} \Omega}>0\right] \sim \frac{\ln L}{L}
$$

as $L \rightarrow \infty$. Thus the probability of finding any direct accessible path to the global maximum is decreasing in the number of loci, but slowly so. Interestingly they also find that a slight modification of the HoC model obtained by fixing the fitness of $\Delta_{\mathcal{L}} \Omega$ to a value corresponding to the quantile value $\alpha_{L}$ yields a threshold function $\alpha_{1}^{\star}(L)=\frac{\ln L}{L}$, such that $\lim _{L \rightarrow \infty} \mathbb{P}\left[\#_{\mathrm{dp} \Omega}>0\right]=1$ for $\alpha_{L}=\alpha_{1}^{\star}(L)-\epsilon_{L}$ and $\lim _{L \rightarrow \infty} \mathbb{P}\left[\#_{\mathrm{dp} \Omega}>0\right]=0$ for $\alpha_{L}=\alpha_{1}^{\star}(L)+\epsilon_{L}$ where $\epsilon_{L}>0$ arbitrary, such that $\lim _{L \rightarrow \infty} L \epsilon_{L}=\infty$. Thus the direct accessibility of the global maximum is, for large enough $L$, mainly constrained by the initial fitness and tends to 1 in particular if the initial genotype is constrained to be the global minimum of the landscape. The limit distribution of direct accessible paths to the global maximum has been further studied in [4.

Berestycki et al. [5] consider arbitrary length accessible paths to the global maximum from its antipodal and find a threshold behavior as well. While $\mathbb{E}\left[\#_{\mathrm{p} \Omega}\right]$ grows exponentially for $\alpha_{L}<\alpha_{2}^{\star}=1-\ln (\sqrt{2}+1)=$ 
$0.11863 \ldots$, it decays exponentially to zero for $\alpha_{L}>\alpha_{2}^{\star}$. Thus $\mathbb{P}\left[\#_{\mathrm{p} \Omega}>0\right]$ for the original $\mathrm{HoC}$ model must be asymptotically bounded from above by $\alpha_{2}^{\star}$. Berestycki et al. conjecture that the expectation "tells the truth", i.e. that $\lim _{L \rightarrow \infty} \mathbb{P}\left[\#_{\mathrm{p} \Omega}>0\right]=1$ for $\alpha_{L}<\alpha_{2}^{\star}$, which would also imply $\lim _{L \rightarrow \infty} \mathbb{P}\left[\#_{\mathrm{p} \Omega}>0\right]=\alpha_{2}^{\star}$ for the original HoC model. This conjecture was proven by Martinsson [47. Computational results for the HoC model with a larger number of alleles, $A>2$, suggest that for any fixed number of alleles $\mathbb{P}\left[\#_{\mathrm{p} \Omega}>0\right]$ converges to values strictly between 0 and 1 as $L \rightarrow \infty$ [102] .

\subsection{Block neighborhood}

The accessibility of the block model has been studied in 78 . Because mutational effects of loci on different blocks are completely statistically independent and fully additive, a path in the BN model is accessible if and only if the restriction of the path onto each block is accessible. Additionally due to this independence of blocks, the global maximum of the full landscape will also be the global maximum on the individual blocks. Thus, $\#_{\mathrm{dp} \Omega}$ will be a product of $\frac{L}{k}$ independent realizations of $\#_{\mathrm{dp} \Omega}$ for HoC landscapes with $k$ loci.

For the probability to find an accessible direct path to the global maximum in particular we have then

$$
\mathbb{P}\left[\#_{\mathrm{dp} \Omega}^{\mathrm{BN}}>0\right]=\mathbb{P}\left[\#_{\mathrm{dp} \Omega}^{\mathrm{HoC}(k)}>0\right]^{\frac{L}{k}}
$$

Therefore at constant $k$, as $L$ increases, this probability decays exponentially to zero. As explained above the direct accessibility for the HoC model goes as $\frac{\ln k}{k}$ for large $k$ and so at $\frac{k}{L}=\alpha$ fixed, asymptotically for large $L$

$$
\mathbb{P}\left[\#_{\mathrm{dp} \Omega}^{\mathrm{BN}}>0\right] \sim\left(\frac{\ln L}{\alpha L}\right)^{\frac{1}{\alpha}}
$$

which is still decreasing to zero, but more slowly. In fact it is closer to the behavior of the HoC model. The functional form is mostly the same, except for the modification by a power of $\frac{1}{\alpha}$ which implies a faster decay than in the HoC model when $\alpha<1$. By the same arguments Eq. 900 holds for $\#_{\mathrm{p} \Omega}$ as well and again the decay at constant $k$ is exponential. At fixed $L / k$ however, using the result for the $\mathrm{HoC}$ model that $\mathbb{P}\left[\#_{\mathrm{p} \Omega}^{\mathrm{HoC}(k)}>0\right]$ actually converges to a non-zero constant for $k \rightarrow \infty, \mathbb{P}\left[\#_{\mathrm{p} \Omega}^{\mathrm{BN}}>0\right]$ also converges to a non-zero constant under this scaling.

Using the same decomposition of the full path into subpaths within blocks, one can see that for the mean number of direct paths a similar equation

$$
\mathbb{E}\left[\#_{\mathrm{dp} \Omega}^{\mathrm{BN}}\right]=\frac{L !}{k ! \frac{L}{k}} \mathbb{E}\left[\#_{\mathrm{dp} \Omega}^{\mathrm{HoC}(k)}\right]^{\frac{L}{k}}=\frac{L !}{k ! \frac{L}{k}}
$$



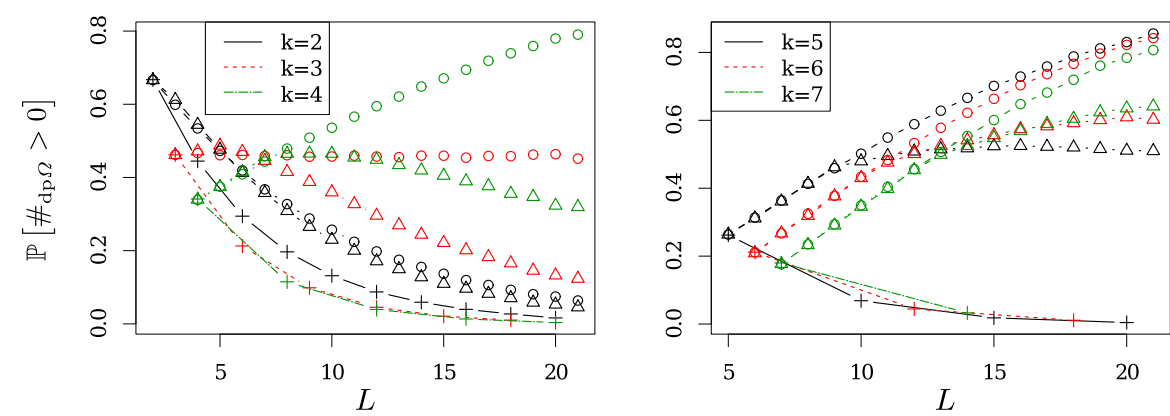

Fig. 7 Accessibility of the global maximum in some NK models. Number of loci $L$ on the $\mathrm{x}$-axis and $\mathbb{P}\left[\#_{\mathrm{dp} \Omega}>0\right]$ on the y-axis for different values of $k$. Different NK structures are determined by symbols used: Triangles for AN, circles for uRN and crosses for BN. Adapted from [78 and based on simulation results using Gaussian base fitness.

holds. The combinatorial factor describes the number of ways in which each set of direct accessible paths on blocks can be combined into a direct accessible path on the full landscape. In fact every realization of $\#_{\mathrm{dp} \Omega}^{\mathrm{BN}}$ must be an integer multiple of this factor. Thus $\mathbb{E}\left[\#_{\mathrm{dp} \Omega}^{\mathrm{BN}}\right]$ increases super-exponentially both under constant $k$ scaling and when $k$ increases proportionally to $L$. Due to the product structure, the actual distribution of $\#_{\mathrm{dp} \Omega}^{\mathrm{BN}}$, scaled by Eq. 92 and conditioned on being larger than 0 , will at constant $k$ be asymptotically log-normal 78.

In summary, the BN model landscape has a large mean number of direct accessible paths, but this is actually hiding the fact that most landscape realizations do not contain a single such path. However, if accessible paths exist, then the multiplicative structure guarantees that there are many. The decay of accessibility is much faster than in the $\mathrm{HoC}$ model for constant $k$ and moderately faster than in the HoC model for proportionate scaling $k \sim L$.

\subsection{Locally bounded NK structures are not traversable}

In the following we consider a large class of NK structures and their asymptotic traversability in the limit of large $L$ and constant $k$.

Let $\rho_{l}$ be the number of loci that have graph distance 4 or less to locus $l$ in the NK structure hypergraph. We then say an NK structure is (distance 4) locally bounded if the mean of $\rho_{l}$ over all $l$ and with respect to realizations of randomized structures has finite limit superior. In particular structures which are regular, uniform and have at most a linearly growing number of edges in $L$ are locally bounded (for arbitrary distances) in the limit $L \rightarrow$ $\infty$ at constant $k$. This holds, because the number of immediate neighbors of $l$ cannot be larger than the number of NK edges it is associated with times the number of elements in these edges. Examples of such structures are the AN, BN and urRN models. The boundedness property also holds for the $(\mathrm{u})(\mathrm{r}) \mathrm{RN}$ models at constant $k$, because the degree distributions of loci become effectively independent and all their moments converge to $L$ - 
independent values. The SN and MF models are however not locally bounded because each locus can reach every other locus in two steps for the SN model and in one step for the MF model. If $k$ is diverging as $L \rightarrow \infty$, then no uniform or regular NK structure can be locally bounded, because each locus is either a member of one edge with a diverging number of elements or a member of a diverging number of edges with at least one other member.

In 19,20 direct accessibility of the global maximum has been studied for the uRN model via simulations. Further simulation data can be found in 78 , for the uRN model, as well as for the AN model. As the BN model is also a representative of the class of locally bounded structures, one might have expected qualitatively similar behavior for these structures. However the AN and uRN models seem to show a more complex behavior in the simulated parameter range, see Fig. 7. In particular, the simulations indicate that accessibility increases with increasing $L$ for the uRN and AN models, at least for sufficiently large $k$. Despite this apparent non-universal trend, it can be shown rigorously that the probability for the existence of traversing paths decays exponentially in $L$ for all locally bounded NK structures [7]. A short summary of the proof will be given here.

Consider two loci $l$ and $m$. There are four allele configurations for these two loci under any given background. They span a 2-dimensional hypercube, i.e. a square. For a given background the associated fitness values can be in one of $4 !=24$ orderings. However reducing some symmetries there are only three different types of fitness graphs, see Fig. 8. Either both sets of parallel arrows are oriented the same way, or only one of the two pairs is, or none. The first case is the one without sign epistasis. The second case identifies a sign epistatic dependence of one locus on the other but not the other way around. And finally the last case shows reciprocal sign epistasis, i.e. sign epistasis between $l$ and $m$ in both directions. This is the only case where the two-locus fitness landscape displays two local maxima and minima, and in fact reciprocal sign epistasis is a necessary condition for the existence of multiple maxima for any number of loci $[70$. Additionally the square becomes non-traversable under reciprocal sign epistasis, because there is no accessible path from any corner to the antipode and the two loci cannot be mutated one after another on an accessible path.

In general, reciprocal sign epistasis between two loci is limited to a particular genetic background, a situation that we refer to as local reciprocal sign epistasis. A third locus on the background may be mutated in-between $l$ and $m$ and thus allow the pathway to cross the square anyway. Strict constraints on the traversability of the full landscape arise, however, if the reciprocal fitness ordering on the $l / m$-square is preserved for all backgrounds. Then mutations in the background cannot influence the direction of fitness effects on $l$ and $m$, i.e. $l$ and $m$ are not sign epistatic with respect to any other locus under any background. We call this global reciprocal sign epistasis (GRSE). It is identified by reciprocal sign epistasis between $l$ and $m$ on all backgrounds, as well as lack of sign epistatic dependence of $l$ and $m$ on any other locus on any background. The existence of a single GRSE locus pair is sufficient to make the landscape non-traversable by direct or indirect 

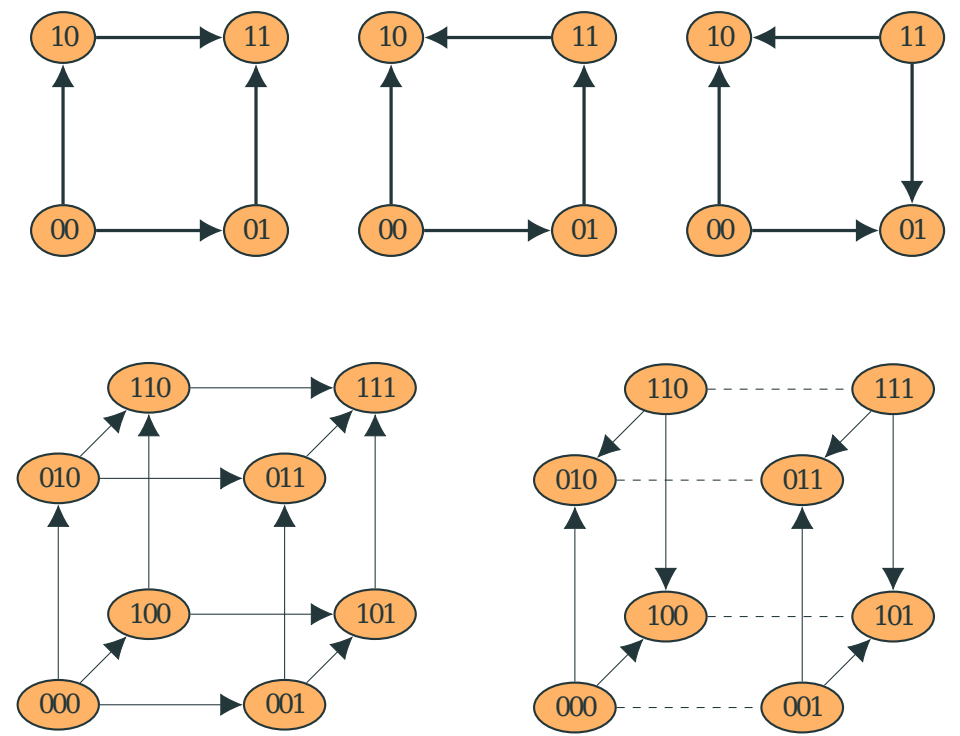

Fig. 8 Top: A single square in the fitness graph, without sign epistasis (left), with non-reciprocal sign epistasis (middle) and reciprocal sign epistasis (right). Bottom: Example of fitness graphs on three loci without any sign epistasis (left) and with global reciprocal sign epistasis (right). Each axis corresponds to one of the loci, and arrows point towards increasing fitness. On the right hand side loci 1 and 2 are globally reciprocal, as determined by the criterion that at any given point either both mutations are deleterious or both are beneficial. Additionally, as required by our definition of global reciprocal sign epistasis, the direction of arrows for loci 1 and 2 is identical for every state of locus 3 , i.e. the pattern in the $1-2$ plane is simply translated along the 3-direction. Note that the direction of arrows for mutations on 3 does not influence this property, and these arrows are therefore not specified. Given global reciprocal sign epistasis there can be no accessible path on the hypercube crossing the loci 1 and 2 simultaneously, and as a consequence one quarter of the nodes of the graph are always unexplorable for an adaptive walk, irrespective of its starting point.

paths, because the locus pair may never be mutated into the antipodal state together. This then also implies $\#_{\mathrm{p} \Omega}=\#_{\mathrm{dp} \Omega}=0$.

In locally bounded NK structures at constant $k$ as $L \rightarrow \infty$, the probability for existence of a GRSE pair of loci approaches unity exponentially fast in $L$. Two loci can only be global reciprocal sign epistatic, or epistatic at all, if they share at least one NK edge. But if they do share at least one NK edge, then there is a probability strictly between 0 and 1 for global reciprocal sign epistasis to occur. For example there is a non-zero probability that $l$ and $m$ are globally reciprocal on the shared NK edge partial landscape (which is simply $\mathrm{HoC}$ ) and that at the same time the smallest fitness difference on the NK edge containing $l$ and $m$ is larger than the largest fitness difference on all of the other NK edges containing either $l$ or $m$. Of course the exact probability depends on the configuration of the NK structure around $l$ and $m$. However only the NK edges containing $l$ and $m$ are of relevance. Fitness values on other edges cannot contribute to fitness differences of mutations on 

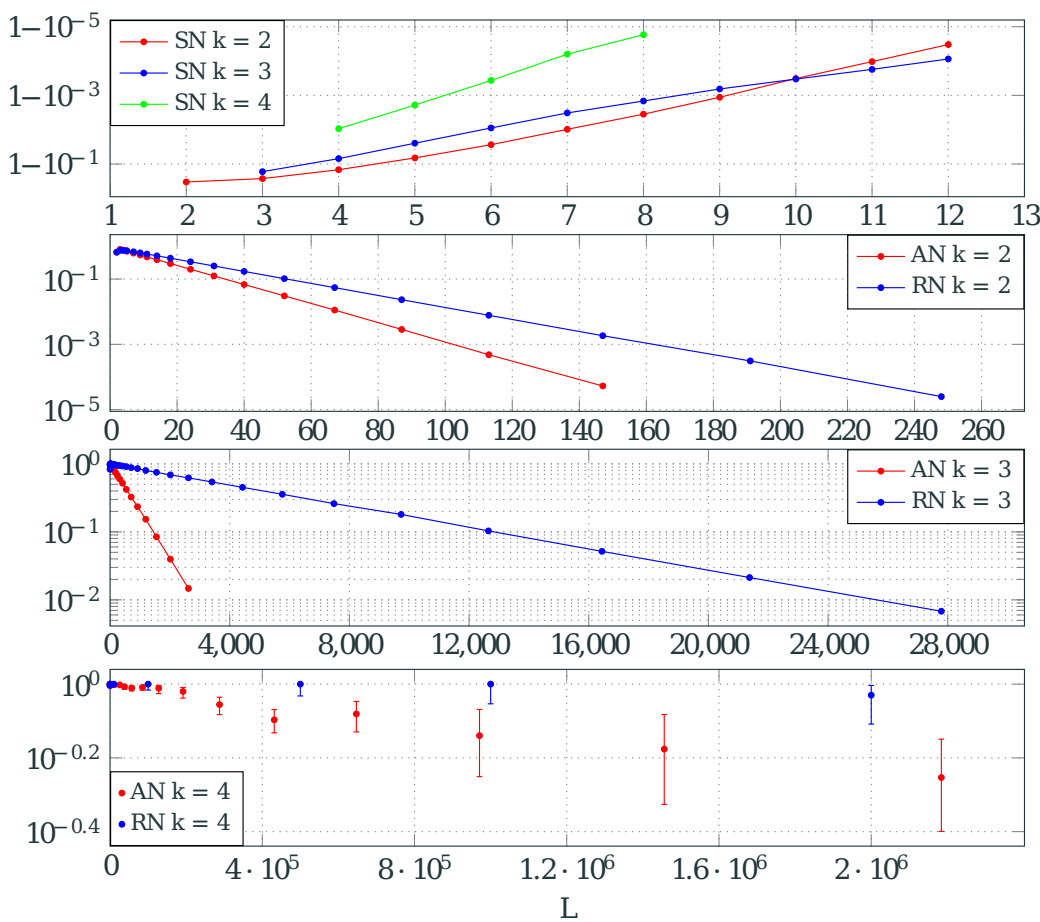

Fig. 9 Simulation results for the probability not to find any global reciprocal sign epistatic pair of loci in the SN, AN and uRN models as a function of the number of loci and for different $k$. The number of simulation runs per data point vary, but the error bars indicate the 95\% Clopper-Pearson confidence interval for the estimate. In the upper panels, error bars would be too small to be distinguishable from the data points. In the $\mathrm{AN}$ and $\mathrm{uRN}$ models, which are locally bounded structure choices, the probability decreases exponentially to zero for all $k \geq 2$. This means that for large number of loci and fixed mean connectivity $k$, there will asymptotically almost surely be pairs of loci that are reciprocally sign epistatic on all backgrounds, such that adaptively accessible paths crossing the whole landscape become impossible. The SN model, as a non-locally bounded alternative structure, behaves completely different. The probability not to find global reciprocal sign epistasis seems to approach unity as $L \rightarrow \infty$, resulting in no possible statement on the number of accessible paths one way or another. A Gaussian base fitness distribution was used in all cases.

$l$ and $m$. Thus the subgraph of the NK structure around $l$ and $m$ determines the probability of $l$ and $m$ being global reciprocal sign epistatic. Two pairs $l_{1}, m_{1}$ and $l_{2}, m_{2}$ of loci are then independent in their property of GRSE if they do not share any NK edges at all, i.e. if they lie in NK structure graph distance of at least 2 .

In locally bounded structures it is possible to find a non-zero fraction of loci for which the number of loci $\rho_{l}$ at distance 4 or less is smaller than some $L$-independent constant. By way of elimination one can choose a linearly growing subset of these loci, such that they are additionally mutually separated by distance at least 4 . These loci are then mutually independent in their property of GRSE. As their degree must also be bounded by an $L$ - 
independent constant, there are only a finite number of structures possible in their immediate neighborhood. Thus the infimum over the individual probabilities of GRSE for all possible configurations is also strictly larger than zero. Combining the non-zero infimum with the linearly growing number of independent realizations, the probability that there is no global reciprocal sign epistasis at all is at most a value smaller than 1 taken to the power of a non-zero fraction of $L$. Thus the probability to find GRSE approaches 1 at least exponentially and the traversability decreases at least exponentially to zero, as do $\mathbb{P}\left[\#_{\mathrm{dp} \Omega}>0\right]$ and $\mathbb{P}\left[\#_{\mathrm{p} \Omega}>0\right]$.

This is consistent with the more precise result for the BN model, in which the traversability decreases exactly exponentially without any polynomial correction and with a growth rate derived from the corresponding property in the HoC model. The argument cannot however be applied to the SN model, as this structure is not locally bounded.

For the AN and uRN model these results seem to contradict the simulation results presented earlier, where it appeared that direct accessibility of the global maximum converges to 1 instead of 0 in the simulated range of $L$, for sufficiently large $k$. However this turns out to be a small system size effect only. The argument above was purely qualitative. The actual decay rates for accessibility may scale extremely strongly with $k$, and in fact it becomes difficult to find GRSE in either model even at relatively large $L$ already for small $k$ (Fig. 9).

\section{Adaptive walks}

Adaptive walks are a simplified class of evolutionary dynamics that arise from a more comprehensive description, as provided, e.g., by the WrightFisher and Moran models 64, in the limit of strong selection and weak mutation (SSWM) 25,58. The weak mutation condition states that the supply of beneficial mutations is low enough to ensure that each newly arising mutation either fixes or goes extinct before another mutation appears. Apart from the brief periods during which a clone of mutants is on its way to fixation or extinction, the population is then almost always monomorphic. The precise form of the fixation probability depends on the underlying population dynamical model, but often the Kimura formula 38

$$
p_{\text {fix }}^{N}(s)=\frac{1-e^{-2 s}}{1-e^{-2 N s}}
$$

is employed, where $s$ is the fitness difference between the mutant and the resident type and $N$ denotes the population size. Within the SSWM approximation, strong selection refers to the condition that the magnitude of typical fitness differences $s$ scaled with the population size $N$ is large, $N|s| \gg 1$. According to $(93$ this implies that only beneficial mutations that increase fitness $(s>0)$ have a chance of going to fixation in the population. Thus in the SSWM regime, the population can be regarded as a point in genotype space that moves along paths of increasing fitness in single mutational steps. These are exactly the accessible pathways that were discussed in the 
preceding section, but the viewpoint here is different: Rather than just asking whether or not accessible pathways exist, the adaptive walk models also address the likelihood that a given path is actually traversed by the evolving population.

In the adaptive walk setting the waiting times for mutation and fixation events are ignored and the process is reduced to a discrete time Markov chain on the set of genotypes. It is evident from the derivation sketched above that the transition probability $T(\sigma \rightarrow \theta)$ between two adjacent genotypes is given by the fixation probability of the $\theta$-mutant in the $\sigma$-background normalized by the sum over the fixation probabilities of all fitter genotypes that are reachable from $\sigma$,

$$
T(\sigma \rightarrow \theta)=\frac{p_{\text {fix }}^{\infty}(F(\theta)-F(\sigma))}{\sum_{\tau \in \mathcal{N}_{+}(\sigma)} p_{\text {fix }}^{\infty}(F(\tau)-F(\sigma))},
$$

where $\mathcal{N}_{+}(\sigma)=\left\{\Delta_{l} \sigma \mid l \in \mathcal{L}, \Delta_{l} F(\sigma)>0\right\}$ is the set of mutational neighbors of $\sigma$ that have higher fitness, and it is understood that $p_{\text {fix }}^{\infty}(s)=0$ for $s<0$.

Three limiting cases of the dynamics Eq. (94) that arise from specific assumptions about the scale of the fitness differences are of particular interest. First, if all fitness differences are small in absolute terms, then the linear approximation $p_{\text {fix }}^{\infty}(s) \approx 2 s$ can be employed and the transition probabilities become proportional to the (positive) fitness differences. This is the setting originally considered by Gillespie and Orr [24,25, 58, and further studied in 32, 33, 49,79. Conversely, if all (positive) fitness differences are large, then $p_{\text {fix }}^{\infty} \rightarrow 1$ for all beneficial mutants and $T(\sigma \rightarrow \theta) \rightarrow\left|\mathcal{N}_{+}(\sigma)\right|^{-1}$ independent of $\theta$, which implies that any fitter neighboring genotype is chosen with equal probability. This defines the random adaptive walk introduced by Kauffman and Levin 35. Finally, if the fitness differences are very inhomogeneous, such that one of them is much larger than all the others, then the Markov chain defined by $(94)$ moves deterministically to the neighboring genotype of largest fitness. This limit of greedy adaptation was also addressed by Kauffman and Levin 35 and studied in detail on uncorrelated fitness landscapes by Orr 59 . So-called reluctant adaptive walks that move deterministically to the element of $\mathcal{N}_{+}(\sigma)$ that has lowest fitness have also been considered 54, though they seem to lack a natural interpretation in the framework of the general model defined by Eq. (94).

Importantly, the trajectories of random, greedy and reluctant adaptive walks are fully specified by the rank ordering of the fitness values. This is a property that they share with the other probes of fitness landscape ruggedness, local maxima and accessible pathways, that have been discussed in the preceding sections. The primary measure of ruggedness is the average number of steps required for the walk to reach a local fitness maximum from a random starting genotype, a quantity that will be referred to as the length of the walk $\ell$. The known results for the walk length on the uncorrelated HoC landscape to leading order in $L$ are summarized in Table 2, Greedy walks reach a local maximum after a finite (small) number of steps, whereas the walk length diverges logarthmically in $L$ for random adaptive walks and linearly for reluctant walks. 


\begin{tabular}{l|l|l|l} 
Walk type & Length $\ell$ & Height $1-\kappa / L$ & References \\
\hline Greedy & $e-1$ & $\kappa=0.4003 \ldots$ & {$[54,59$} \\
Random & $\ln L$ & $\kappa=0.6243 \ldots$ & {$[18,44,54$} \\
Reluctant & $L / 2$ & $\kappa=1$ & {$[52,54$}
\end{tabular}

Table 2 Properties of adaptive walks on the House-of-Cards landscape with fitness values distributed uniformly on the interval $[0,1]$.

Analytical results for walk lengths on correlated fitness landscapes are relatively scarce, but some progress has recently been achieved for walks on Rough Mount Fuji landscapes [1,51, a class of models defined by a weighted superposition of an additive fitness landscape and an uncorrelated random (HoC) landscape 65, 63, 62. For the discussion of adaptive walks on NK landscapes we start from the observation that the walk length is additive over blocks for the block neighborhood [54,66,79], and therefore

$$
\ell_{\mathrm{BN}}=\frac{L}{k} \ell_{\mathrm{HoC}}(k)
$$

holds as an exact relation. The dependence on the walk type enters through the $\mathrm{HoC}$ walk length $\ell_{\mathrm{HoC}}$, the asymptotics of which can be read off from Table 2. Although Eq. (95) is not quantitatively correct for other interaction structures, it captures several important features of the walk length in the NK model. In particular for fixed $k$ the walk length grows linearly in $L$, and the ordering among different walk types corresponds to that obtained for the HoC landscape 54.

An argument due to Weinberger 92 links the linear dependence of the walk length on the number of loci $L$ to the exponential decay of the density of fitness maxima $\pi_{\max } \sim\left(\lambda_{k}\right)^{L}$. Since the total number of genotypes is $2^{L}$ and the number of maxima $\#_{\max } \sim\left(2 \lambda_{k}\right)^{L}$, the average "basin of attraction" of a maximum contains $2^{L} / \#_{\max }=\lambda_{k}^{-L}$ sequences. Such a basin can be visualized as a volume with a diameter $D$ given by

$$
D=L\left|\log _{2}\left(\lambda_{k}\right)\right|
$$

and Weinberger claims that $D / 2$ provides a lower bound on the length of any adaptive walk, in particular on the length of a greedy (or gradient) walk. Comparison with the exact relation Eq. (95) shows that the latter statement is not quite true. Since the greedy $\mathrm{HoC}$ walk length has a finite limit $e-1$ for $k \rightarrow \infty$ whereas $\ln \left(\lambda_{k}^{\mathrm{BN}}\right) \sim-(\ln k) / k$, we see that $\ell_{\mathrm{BN}} \sim$ $L / k \ll D \sim(L / k) \log _{2} k$ for large $k$. This discrepancy may be related to the strong clustering of local maxima that has been observed in particular for the BN neighborhood; we will return to this point below in Section 6.3.

Nevertheless the negative correlation between the adaptive walk length and the density of local maxima suggested by Eq. $(96)$ is confirmed by detailed simulations of different types of walks on $\mathrm{NK}$ landscapes with different interaction structures [54. Walk lengths are always shortest on BN landscapes, intermediate on AN landscapes and longest on RN landscapes, and the walk length is positively correlated with the rank of the interaction 
scheme. Whether the universality results obtained for $\lambda_{k}$ can be extended to adaptive walk lengths remains an open question for future work.

Within the framework of abstract landscape theory it has been postulated that the length of adaptive walks should be related to the correlation length $\xi$ of the fitness landscape, which can be generally defined in terms of the distance correlation function by $73,80,81$

$$
\xi=\sum_{d=0}^{\infty} \rho(d) .
$$

Inserting the expression Eq. (15) for the classical NK structures one finds the simple result

$$
\xi=\frac{L+1}{k+1} .
$$

This is of the same leading order as, but generally smaller than Eq. (95), which is expected to be a lower bound on the adaptive walk lengths (note that $\ell_{\mathrm{HoC}} \geq e-1>1$ according to Table 2 .

Apart from the length of an adaptive walk it is also of interest to consider the height reached, i.e. the fitness value of the local maximum at which the walk terminates. Results for the height of adaptive walks on HoC landscapes are summarized in Table 2, where fitness values are assumed for concreteness to be uniformly distributed on the unit interval. On this scale the expected fitness value of a randomly chosen local maximum is $1-1 /(L+2) \approx 1-1 / L$ for large $L$. It can thus be seen from Table 2 that random and greedy adaptive walks terminate at local maxima of atypically high fitness, and that greedy walks are more efficient than random walks in reaching exceptionally high peaks. Whether or not the fitness peaks located by an adaptive walk are typical is of interest in situations where walks are used to explore empirical fitness landscapes that are too large for local maxima to be enumerated exhaustively [3, 41.

A numerical study of walk heights on NK fitness landscapes revealed a surprisingly complex dependence on the interaction structure and the type of the walks 54. For the BN and AN structures the greedy (reluctant) walks are most (least) efficient in locating high fitness peaks, as might be expected from the results for the HoC landscapes, but for the RN structure this order can be reversed in a range of $k$. At fixed $k$ the walk height generally increases with the rank of the interaction structure.

\section{Discussion and conclusion}

\subsection{Biological implications}

Conflicting intuitions about the topography of fitness landscapes have been the cause of debate in evolutionary theory ever since the concept first appeared [21,83. Whereas Sewall Wright argued that these landscapes are likely to possess "innumerable peaks...which are separated by valleys" and stressed the need to understand how evolution is able to find its way "from lower to 
higher peaks" [100], his opponent Ronald Fisher thought that the problem would not present itself because of the high dimensionality of genotype space 72. At its mathematical core, Fisher's argument is a statement about the overwhelming likelihood of extrema of high-dimensional differentiable functions to be saddle points rather than maxima or minima and as such, it ignores the specific, discrete structure of the space of genotypes.

An important role of the probabilistic fitness landscape models considered in this review is that they allow us to phrase and answer questions about the generic structure of genotypic fitness landscapes in precise mathematical terms 89. In a certain sense, they show that Wright and Fisher were both right: Although it is true that the fraction of fitness peaks among all genotypes, $\pi_{\max }$, generally decreases with increasing genotype dimensionality, this is more than offset by the exponential growth of the total number genotypes in such a way that the number of peaks \# max also grows exponentially. We have seen that, in the NK models, $\pi_{\max }$ decays exponentially or algebraically in $L$ depending on whether the epistasis parameter $k$ is kept constant or scaled to infinity, and the SN structure exemplifies the kind of epistatic interactions that are required for $\#_{\max }$ not to diverge when $L \rightarrow \infty$.

The general biological message of our work is that the ruggedness of a fitness landscape depends not only on the amount of genetic interactions, but also on how these interactions are organized. Whereas the fact that epistastic interactions are ubiquitous and often lead to complex fitness landscapes is now widely appreciated, researchers are only beginning to pose more refined questions regarding the structure of the interactions. For example, several recent articles have addressed the prevalence and evolutionary role of higherorder interactions that cannot be reduced to contributions from pairs of loci 10, 76,94 .

Within the class of NK-models, the parameter $k$ specifies the highest order of interactions that are present in the system 50. Comparing different NK interaction structures at a given $k$ thus amounts to exploring effects that go beyond the interaction order and involve more subtle aspects of genetic architecture. In this regard, our analysis shows that two structural paradigms that can be regarded as extremes in a spectrum of possible architectures, the perfectly modular BN structure and the strongly hierarchical SN structure, also represent extremes with respect to the ruggedness of the resulting fitness landscape: The BN landscape has the largest number of fitness maxima, whereas the number of maxima in the SN landscape remains finite for $L \rightarrow$ $\infty$. The (deterministic) AN structure and the (random) RN structure are intermediate between these two limits, but AN landscapes are more rugged than RN landscapes for large $k$.

We hope that these analyses can serve as a starting point for further exploration of other, empirically motivated interaction schemes. Recent highthroughput experiments on protein fitness landscapes suggest that it is principally feasible to extract the interaction structure by determining the type of epistasis between pairs of loci [3,71]. 
6.2 Universality

One reason for the broad appeal of the NK models in the description of fitness landscapes lies in their promise of universality, in that quantities like $\pi_{\max }$ and $\#_{\max }$ depend only on the gross parameters $L$ and $k$ (at least when both are large), and are robust against changes in the detailed interaction structure and the underlying base fitness distribution. Our new analyses presented in Sec. 3 confirm that universality holds, but it is more restricted than previously appreciated. Specifically, we find evidence for two distinct universality classes characterized by different asymptotic behaviors of $\ln \lambda_{k}=\lim _{L \rightarrow \infty} L^{-1} \ln \pi_{\max }$ for large $k$. It should nevertheless be emphasized that the degree of universality with respect to the base fitness distribution $p_{f}$ is very strong, as evidenced by the results of 43 ] as well as by our computation for the RN model in Sec. 3.2.4. In this respect the NK landscapes differ markedly from the Rough Mount Fuji (RMF) model, another class of tunably rugged fitness landscapes for which an explicit expression for $\pi_{\max }$ can be derived, and where the asymptotic behavior of this quantity is dominated by the tail properties of $p_{f}$ [51].

Whereas the number of fitness maxima remains the most commonly used quantifier of ruggedness, the statistics of accessible pathways and adaptive walks reviewed in Sections 4 and 5 address the searchability of fitness landscapes in a more direct way. Following the terminology first introduced by Weinreich and collaborators, a pathway is called accessible if it is monotonically increasing in fitness, and a landscape is accessible if the global fitness maximum can be reached through an accessible pathway starting from its antipodal point $[9,19,95,93$. The central result outlined in Sec. 4.4 states that the probability for an NK fitness landscape to be accessible decays exponentially in $L$ whenever the interaction structure is locally bounded, a property that applies to all commonly used structures. Somewhat counterintuitively, this implies that NK landscapes are much less accessible than uncorrelated HoC landscapes, for which the decay is only algebraic and moreover accessibility can be boosted simply by choosing a starting point of low fitness [4, 29]. This shows that local fitness peaks and accessible pathways reflect distinct properties of fitness landscapes that cannot easily be subsumed into a single notion of ruggedness. Importantly, the exponential decay of accessibility was not seen in earlier numerical work on the NK model because of the extreme scarcity of the crucial GRSE motifs for large $k$.

\subsection{Outlook}

The results described in Sec. 3 suggest a number of promising avenues for future work on NK fitness landscapes. On the side of mathematical analysis, a more rigorous treatment of the joint limit $(L, k \rightarrow \infty$ at fixed $\alpha=k / L)$ for the $\beta=2$ universality class comprising the MF and RN models would be desirable. Also the intriguing role of the $\mathrm{BN}$ model in providing a possibly universal upper bound on the number of maxima among all interaction structures and base fitness distributions should be elucidated. Finally, it seems important to direct the attention to the way fitness maxima are organized 
in sequence space, rather than just focusing on their sheer number. A numerical investigation reported in [54] found that local maxima are strongly clustered, and the degree of clustering is highly dependent on the interaction structure. A better understanding of the organization of maxima would also be helpful in strengthening the link between static landscape properties and the dynamics of adaptive walks evolving on the landscape, which is so far quite sketchy (see Sec. 5). A useful tool for such an analysis is a network approach where the vertices are fitness maxima and the links quantify the overlap between their respective basins of attraction [85.

Among the plethora of research problems that present themselves beyond the specific context of NK models, we here choose to point the reader to the study of time-dependent fitness landscapes which are sometimes referred to as fitness seascapes 48. Natural fitness landscapes are never entirely static, and time-dependent effects are crucial for the explanation of fundamental evolutionary phenomena such as the selective advantage of recombination [55. In 1999, Wilke and Martinetz introduced a time-dependent variant of the NK model 98, which subsequently was picked up by the glass physics community 31 and is meanwhile used routinely for the description of periodically stressed disordered solids [17. This example shows that the transfer of concepts across the interface between evolutionary biology and statistical physics can go both ways, and that further exchanges in this area can be expected to produce surprising and innovative results.

Acknowledgments. We thank David Dean for useful discussions, and an anonymous reviewer for constructive remarks on the manuscript. JK acknowledges the kind hospitality of the MPI for Physics of Complex Systems (Dresden) and the Kavli Institute for Theoretical Physics (Santa Barbara) during the completion of the paper. This research was supported by DFG within SFB 680 Molecular basis of evolutionary innovations and SPP1590 Probabilistic structures in evolution, and in part by the National Science Foundation Grant No. NSF PHY-1125915, NIH Grant No. R25GM067110, and the Gordon and Betty Moore Foundation Grant No. 2919.01.

\section{References}

1. Aita, T., Uchiyama, H., Inaoka, T., Nakajima, M., Kokubo, T., Husimi, Y.: Analysis of a local fitness landscape with a model of the rough Mt. Fuji-type landscape: Application to prolyl endopeptidase and thermolysin. Biopolymers 54(1), 64-79 (2000)

2. Altenberg, L.: NK fitness landscapes. In: T. Bäck, D.B. Fogel, Z. Michalewicz (eds.) Handbook of Evolutionary Computation. IOP Publishing Ltd and Oxford University Press (1997)

3. Bank, C., Matuszewski, S., Hietpas, R.T., Jensen, J.D.: On the (un)predictability of a large intragenic fitness landscape. Proc. Nat. Acad. Sci. USA 113, 14,085-14,090 (2016)

4. Berestycki, J., Brunet, É., Shi, Z.: The number of accessible paths in the hypercube. Bernoulli 22, 653-680 (2016)

5. Berestycki, J., Brunet, É., Shi, Z.: Accessibility percolation with backsteps. ALEA, Lat. Am. J. Probab. Math. Stat. 14, 45-62 (2017) 
6. Buzas, J., Dinitz, J.: An analysis of NK landscapes: Interaction structure, statistical properties and expected number of local optima. IEEE Transactions on Evolutionary Computation 18(6), 807-818 (2014)

7. Campos, P.R.A., Adami, C., Wilke, C.O.: Optimal adaptive performance and delocalization in NK fitness landscapes. Physica A: Statistical Mechanics and its Applications 304, 495-506 (2002)

8. Campos, P.R.A., Adami, C., Wilke, C.O.: Optimal adaptive performance and delocalization in NK fitness landscapes (Erratum). Physica A: Statistical Mechanics and its Applications 318, 637 (2003)

9. Carneiro, M., Hartl, D.L.: Adaptive landscapes and protein evolution. Proc. Nat. Acad. Sci. USA 107, 1747-1751 (2010)

10. Crona, K., Gavryushkin, A., Greene, D., Beerenwinkel, N.: Inferring genetic interactions from comparative fitness data. eLife 6, e28,629 (2017)

11. Crona, K., Greene, D., Barlow, M.: The peaks and geometry of fitness landscapes. J. Theor. Biol. 318, 1-10 (2013)

12. Dean, D.S.: Metastable states of spin glasses on random thin graphs. Eur. Phys. J. B 15, 493-498 (2000)

13. DePristo, M.A., Hartl, D.L., Weinreich, D.M.: Mutational reversions during adaptive protein evolution. Mol. Biol. Evol. 24, 1608-1610 (2007)

14. Durrett, R., Limic, V.: Rigorous results for the NK model. Ann. Prob. 31, $1713-1753$ (2003)

15. Evans, S.N., Steinsaltz, D.: Estimating some features of NK fitness landscapes. Ann. Appl. Probab. 12, 1299-1321 (2002)

16. Ferretti, L., Schmiegelt, B., Weinreich, D., Yamauchi, A., Kobayashi, Y., Tajima, F., Achaz, G.: Measuring epistasis in fitness landscapes: The correlation of fitness effects of mutations. J. Theor. Biol. 396, 132-143 (2016)

17. Fiocco, D., Foffi, G., Sastry, S.: Encoding of memory in sheared amorphous solids. Phys. Rev. Lett. 112, 025,702 (2014)

18. Flyvbjerg, H., Lautrup, B.: Evolution in a rugged fitness landscape. Phys. Rev. A 46, 6714-6723 (1992)

19. Franke, J., Klözer, A., de Visser, J.A.G.M., Krug, J.: Evolutionary accessibility of mutational pathways. PLoS Comp. Biol. 7(8), e1002,134 (2011)

20. Franke, J., Krug, J.: Evolutionary accessibility in tunably rugged fitness landscapes. J. Stat. Phys. 148, 705-722 (2012)

21. Gavrilets, S.: Fitness Landscapes and the Origin of Species. Princeton University Press, Princeton (2004)

22. Genz, A.: Numerical computation of multivariate normal probabilities. Journal of Computational and Graphical Statistics 1(2), 141-149 (1992)

23. Genz, A., Bretz, F., Miwa, T., Mi, X., Leisch, F., Scheipl, F., Hothorn, T. mvtnorm: Multivariate Normal and t Distributions (2017). R package version $1.0-6$

24. Gillespie, J.H.: A simple stochastic gene substitution model. Theor. Popul. Biol. 23, 202-215 (1983)

25. Gillespie, J.H.: Molecular evolution over the mutational landscape. Evolution 38, 1116-1129 (1984)

26. de Haan, L., Ferreira, A.: Extreme Value Theory: An Introduction (Springer Series in Operations Research). Springer (2006)

27. Haldane, J.B.S.: A mathematical theory of natural selection, Part VIII: Metastable populations. Proc. Cambridge Philos. Soc. 27, 137-142 (1931)

28. Hartl, D.L.: What can we learn from fitness landscapes? Curr. Opin. Microbiol. 21, 51-57 (2014)

29. Hegarty, P., Martinsson, A.: On the existence of accessible paths in various models of fitness landscapes. Ann. Appl. Probab. 24, 1375-1395 (2014)

30. Hwang, S., Park, S.C., Krug, J.: Genotypic complexity of Fisher's geometric model. Genetics 206, 1049-1079 (2017)

31. Isner, B.A., Lacks, D.J.: Generic rugged landscapes under strain and the possibility of rejuvenation in glasses. Phys. Rev. Lett. 96, 025,506 (2006)

32. Jain, K.: Number of adaptive steps to a local fitness peak. Europhys. Lett. 96, 58,006 (2011) 
33. Jain, K., Seetharaman, S.: Multiple Adaptive Substitutions During Evolution in Novel Environments. Genetics 189, 1029-1043 (2011)

34. Kanwal, R.P.: Linear Integral Equations: Theory \& Technique (Modern Birkhäuser Classics). Birkhäuser (2012)

35. Kauffman, S., Levin, S.: Towards a general theory of adaptive walks on rugged landscapes. Journal of Theoretical Biology 128(1), 11-45 (1987)

36. Kauffman, S.A.: The Origins of Order. Oxford University Press, Oxford (1993)

37. Kauffman, S.A., Weinberger, E.D.: The NK model of rugged fitness landscapes and its application to maturation of the immune response. J. Theor. Biol. 141, 211-245 (1989)

38. Kimura, M.: On the probability of fixation of mutant genes in a population. Genetics 47, 713-719 (1962)

39. Kingman, J.F.C.: A simple model for the balance between selection and mutation. Journal of Applied Probability 15(1), 1-12 (1978)

40. Kondrashov, D.A., Kondrashov, F.A.: Topological features of rugged fitness landscapes in sequence space. Trends in Genetics 31, 24-33 (2015)

41. Kouyos, R.D., Leventhal, G.E., Hinkley, T., Haddad, M., Whitcomb, J.M. Petropoulos, C.J., Bonhoeffer, S.: Exploring the complexity of the HIV-1 fitness landscape. PLoS Genet. 8, e100255,151 (2012)

42. Levinthal, D.A.: Adaptation on rugged landscapes. Management Science 43, 934-950 (1997)

43. Limic, V., Pemantle, R.: More rigorous results on the Kauffman-Levin model of evolution. Ann. Prob. 32, 2149-2178 (2004)

44. Macken, C.A., Hagan, P.S., Perelson, A.S.: Evolutionary walks on rugged landscapes. SIAM Journal on Applied Mathematics 51(3), 799-827 (1991)

45. Macken, C.A., Perelson, A.S.: Protein evolution on rugged landscapes. Proc. Nat. Acad. Sci. USA 86, 6191-6195 (1989)

46. Manukyan, N., Eppstein, M.J., Buzas, J.S.: Tunably rugged landscapes with known maximum and minimum. IEEE Transactions on Evolutionary Computation 20, 263-274 (2016)

47. Martinsson, A.: Accessibility percolation and first-passage site percolation on the unoriented binary hypercube. Preprint arXiv:1501.02206 (2015)

48. Mustonen, V., Lässig, M.: From fitness landscapes to seascapes: nonequilbrium dynamics of selection and adaptation. Trends in Genetics 25, 111-119 (2009)

49. Neidhart, J., Krug, J.: Adaptive walks and extreme value theory. Phys. Rev. Lett. 107, 178,102 (2011)

50. Neidhart, J., Szendro, I.G., Krug, J.: Exact results for amplitude spectra of fitness landscapes. J. Theor. Biol. 332, 218-227 (2013)

51. Neidhart, J., Szendro, I.G., Krug, J.: Adaptation in tunably rugged fitness landscapes: The Rough Mount Fuji Model. Genetics 198, 699-721 (2014)

52. Nowak, S.: Properties of Random Fitness Landscapes and Their Influence on Evolutionary Dynamics. A Journey through the Hypercube. PhD dissertation, Cologne (2015)

53. Nowak, S., Krug, J.: Accessibility percolation on $n$-trees. Europhys. Lett. 101, 66,004 (2013)

54. Nowak, S., Krug, J.: Analysis of adaptive walks on NK fitness landscapes with different interaction schemes. Journal of Statistical Mechanics: Theory and Experiment P06014 (2015)

55. Nowak, S., Neidhart, J., Szendro, I.G., Krug, J.: Multidimensional epistasis and the transitory advantage of sex. PLoS Comp. Biol. 10, e1003,836 (2014)

56. Ohta, T.: The meaning of near-neutrality at coding and non-coding regions. Gene 205, 261-267 (1997)

57. de Oliviera, V.M., Fontanari, J.F., Stadler, P.F.: Metastable states in shortranged $p$-spin glasses. J. Phys. A 32, 8793-8802 (1999)

58. Orr, H.A.: The population genetics of adaptation: the adaptation of DNA sequences. Evolution 56, 1317-1330 (2002)

59. Orr, H.A.: A minimum on the mean number of steps taken in adaptive walks. J. Theor. Biol. 220, 241-247 (2003) 
60. Orr, H.A.: The population genetics of adaptation on correlated fitness landscapes: the block model. Evolution 60, 1113-1124 (2006)

61. Østman, B., Hintze, A., Adami, C.: Impact of epistasis and pleiotropy on evolutionary adaptation. Proc. R. Soc. Lond. Ser. B 279, 247-256 (2012)

62. Park, S.C., Krug, J.: $\delta$-exceedance records and random adaptive walks. J. Phys. A 49, 315,601 (2016)

63. Park, S.C., Neidhart, J., Krug, J.: Greedy adaptive walks on a correlated fitness landscape. J. Theor. Biol. 397, 89-102 (2016)

64. Park, S.C., Simon, D., Krug, J.: The speed of evolution in large asexual populations. J. Stat. Phys. 138, 381-410 (2010)

65. Park, S.C., Szendro, I.G., Neidhart, J., Krug, J.: Phase transition in random adaptive walks on correlated fitness landscapes. Phys. Rev. E 91, 042,707 (2015)

66. Perelson, A.S., Macken, C.A.: Protein evolution on partially correlated landscapes. Proceedings of the National Academy of Sciences of the United States of America 92(21), 9657-9661 (1995)

67. Phillips, P.C.: Epistasis - the essential role of gene interactions in the structure and evolution of genetic systems. Nature Reviews Genetics 9, 855-867 (2008)

68. Poelwijk, F.J., Kiviet, D.J., Weinreich, D.M., Tans, S.J.: Empirical fitness landscapes reveal accessible evolutionary paths. Nature 445, 383-386 (2007)

69. Poelwijk, F.J., Krishna, V., Ranganathan, R.: The context-dependence of mutations: A linkage of formalisms. PLoS Comp. Biol. 12, e1004,771 (2016)

70. Poelwijk, F.J., Tănase-Nicola, S., Kiviet, D.J., Tans, S.J.: Reciprocal sign epistasis is a necessary condition for multi-peaked fitness landscapes. J. Theor. Biol. 272, 141-144 (2011)

71. Pokusaeva, V.O., Usmanova, D.R., Putintseva, E.V., Espinar, L., Sarkisyan, K.S., Mishin, A.S., Bogatyreva, N.S., Ivankov, D.N., Povolotskaya, I.S., Filion, G.J., Carey, L.B., Kondrashov, F.A.: Experimental assay of a fitness landscape on a macroevolutionary scale. Preprint bioRxiv 222778 (2017)

72. Provine, W.B.: Sewall Wright and Evolutionary Biology. University of Chicago Press, Chicago (1986)

73. Reidys, C.M., Stadler, P.F.: Combinatorial landscapes. SIAM Review 44, 3-54 (2002)

74. Richter, H., Engelbrecht, A. (eds.): Recent Advances in the Theory and Application of Fitness Landscapes. Springer, Berlin, Heidelberg (2014)

75. Rowe, W., Platt, M., Wedge, D.C., Day, P.J., Kell, D.B., Knowles, J.: Analysis of a complete DNA-protein affinity landscape. J. R. Soc. Interface 7, 397-408 (2010)

76. Sailer, Z.R., Harms, M.J.: High-order epistasis shapes evolutionary trajectories. PLoS Comp. Biol. 13, e1005,541 (2017)

77. Schmiegelt, B.: Sign epistasis networks. Master thesis, Cologne (2016)

78. Schmiegelt, B., Krug, J.: Evolutionary Accessibility of Modular Fitness Landscapes. Journal of Statistical Physics 154(1), 334-355 (2014)

79. Seetharaman, S., Jain, K.: Length of adaptive walk on uncorrelated and correlated fitness landscapes. Phys. Rev. E 90, 032,703 (2014)

80. Stadler, P.F.: Landscapes and their correlation functions. Journal of Mathematical Chemistry 20, 1-45 (1996)

81. Stadler, P.F., Happel, R.: Random field models for fitness landscapes. J. Math. Biol. 38, 435-478 (1999)

82. Stein, D.L. (ed.): Spin Glasses and Biology. World Scientific, Singapore (1992)

83. Svensson, E.I., Calsbeek, R. (eds.): The adaptive landscape in evolutionary biology. Oxford University Press, Oxford (2012)

84. Szendro, I.G., Schenk, M.F., Franke, J., Krug, J., de Visser, J.A.G.M.: Quantitative analyses of empirical fitness landscapes. J. Stat. Mech.:Theory Exp. P01005 (2013)

85. Tomassini, M., Vérel, S., Ochoa, G.: Complex-network analysis of combinatorial spaces: The NK landscape case. Phys. Rev. E 78, 066,114 (2008)

86. Touchette, H.: The large deviation approach to statistical mechanics. Physics Reports 478(1), 1-69 (2009) 
87. Valente, M.: An NK-like model for complexity. Journal of Evolutionary Economics 24, 107-134 (2014)

88. de Visser, J.A.G.M., Cooper, T.F., Elena, S.F.: The causes of epistasis. Proc. R. Soc. Lond. Ser. B 278, 3617-3624 (2011)

89. de Visser, J.A.G.M., Krug, J.: Empirical fitness landscapes and the predictability of evolution. Nature Reviews Genetics 15, 480-490 (2014)

90. de Visser, J.A.G.M., Park, S.C., Krug, J.: Exploring the effect of sex on empirical fitness landscapes. Am. Nat. 174, S15-S30 (2009)

91. Weinberger, E.D.: Fourier and Taylor series on fitness landscapes. Biol. Cybern. 65, 321-330 (1991)

92. Weinberger, E.D.: Local properties of Kauffman's N-k model: A tunably rugged energy landscape. Phys. Rev. A 44, 6399-6413 (1991)

93. Weinreich, D.M., Delaney, N.F., DePristo, M.A., Hartl, D.L.: Darwinian evolution can follow only very few mutational paths to fitter proteins. Science 312, 111-114 (2006)

94. Weinreich, D.M., Lan, Y., Wylie, C.S., Heckendorn, R.B.: Should evolutionary geneticists worry about higher-order epistasis? Curr. Op. Genet. Develop. 23, 700-707 (2013)

95. Weinreich, D.M., Watson, R.A., Chao, L.: Sign epistasis and genetic constraint on evolutionary trajectories. Evolution 59, 1165-1174 (2005)

96. Welch, J.J., Waxman, D.: The $n k$ model and population genetics. J. Theor. Biol. 234, 329-340 (2005)

97. Whitlock, M.C., Phillips, P.C., Moore, F.B.G., Tonsor, S.J.: Multiple fitness peaks and epistasis. Annu. Rev. Ecol. Systemat. 26, 601-629 (1995)

98. Wilke, C.O., Martinetz, T.: Adaptive walks on time-dependent fitness landscapes. Phys. Rev. E 60, 2154-2159 (1999)

99. Wright, A.H., Thompson, R.K., Zhang, J.: The computational complexity of N-K fitness functions. IEEE Transactions on Evolutionary Computation 4, 373-379 (2000)

100. Wright, S.: The roles of mutation, inbreeding, crossbreeding and selection in evolution. Proc. 6th Int. Cong. Genet. 1, 356-366 (1932)

101. Wu, N.C., Dai, L., Olson, C.A., Lloyd-Smith, J.O., Sun, R.: Adaptation in protein fitness landscapes is facilitated by indirect paths. eLife 5, 16,965 (2016)

102. Zagorski, M., Burda, Z., Waclaw, B.: Beyond the hypercube: evolutionary accessibility of fitness landscapes with realistic mutational networks. PLoS Comp. Biol. 12(12), e1005,218 (2016)

\section{A Asymptotics of $\pi_{\max }^{\mathrm{MF}}$ in the joint limit $k, L \rightarrow \infty$}

We start from Eq. (34). Rescaling $y \rightarrow \frac{\eta y}{\sqrt{2}}$, we rewrite the equation in terms of the CDF of a standard Gaussian distribution $\Phi(y)$ as

$$
\begin{aligned}
\pi_{\max }^{\mathrm{MF}} & =\sqrt{\frac{L \eta^{2}}{4 \pi}} \int d y e^{-L \eta^{2} y^{2} / 4}\left[\frac{1}{2}\left(\operatorname{erf}\left(\frac{y}{\sqrt{2}}\right)+1\right)\right]^{L} \\
& =\sqrt{\frac{\mu}{2 \pi}} \int d y e^{-\mu y^{2} / 2} \Phi(y)^{L},
\end{aligned}
$$

where $\mu \equiv \frac{L \eta^{2}}{2}$ which converges to $\frac{(2-\alpha)}{\alpha}$ in the joint limit as can be seen from Eq. (32).

Interestingly, the only $L$-dependence shown in the above equation appears as an $L$-th power of the CDF $\Phi(y)$, which converges monotonically to unity as $y \rightarrow \infty$. This implies that the conventional saddle point method cannot be applied here due to the absence of a maximum. Instead, we can rely on the extreme value theory by interpreting the term $\Phi(y)^{L}$ as the probability that $L$ randomly sampled standard 
Gaussian random variables are less than $y$. This leads immediately to the limit relation 26]

$$
\Phi\left(\frac{x}{a_{L}}+b_{L}\right)^{L} \rightarrow G(x)(1+o(1))
$$

where $G(x)$ is the Gumbel CDF defined by $G(x)=e^{-e^{-x}}$, and the two scaling factors are given by $a_{L}=\sqrt{2 \ln L}$ and

$$
b_{L}=\sqrt{2 \ln L}-\frac{\ln \ln L+\ln 4 \pi}{2 \sqrt{2 \ln L}} .
$$

After making the change of variable $y=\frac{x}{a_{L}}+b_{L}$, the integral is now of the form

$$
\begin{aligned}
\pi_{\max }^{\mathrm{MF}} & =\frac{1}{a_{L}} \sqrt{\frac{\mu}{2 \pi}} \int d x e^{-\mu\left(\frac{x}{a_{L}}+b_{L}\right)^{2} / 2} \Phi\left(\frac{x}{a_{L}}+b_{L}\right)^{L} \\
& =\frac{1}{a_{L}} \sqrt{\frac{\mu}{2 \pi}} \int d x e^{-\mu\left(\frac{x}{a_{L}}+b_{L}\right)^{2} / 2} G(x)(1+o(1)) .
\end{aligned}
$$

The evaluation of the integral with respect to $x$ is greatly simplified once one notices that the term $\frac{x^{2}}{a_{L}^{2}}$ in the exponent is sub-leading in $L$. Ignoring this term gives

$$
\begin{aligned}
\pi_{\max }^{\mathrm{MF}} & =\frac{1}{a_{L}} \sqrt{\frac{\mu}{2 \pi}} \int d x e^{-\mu\left(b_{L}^{2}+2 \frac{b_{L} x}{a_{L}}\right) / 2} G(x)(1+o(1)) \\
& =\frac{1}{a_{L}} \sqrt{\frac{\mu}{2 \pi}} e^{-\mu b_{L}^{2} / 2} \Gamma(\mu)(1+o(1)),
\end{aligned}
$$

where we have used the identity

$$
\int_{-\infty}^{\infty} G(x) \exp (-M x) d x=\Gamma(M)
$$

for positive $M$. Next, expanding $a_{L}$ and $b_{L}$ and rearranging the terms gives

$$
\begin{aligned}
\pi_{\max }^{\mathrm{MF}} & =\frac{1}{a_{L}} \sqrt{\frac{\mu}{2 \pi}} e^{-\frac{\mu}{2}[2 \ln L-(\ln \ln L+\ln 4 \pi+o(1))]} \Gamma(\mu)(1+o(1)) \\
& =\sqrt{\mu} \frac{(4 \pi \ln L)^{\mu / 2}}{(4 \pi \ln L)^{1 / 2}} \Gamma(\mu) L^{-\mu}(1+o(1)) .
\end{aligned}
$$

As expected from the formal analysis in Sec. 3.2.2 the leading order behavior is given by a power law with exponent $\mu=(2-\alpha) / \alpha$. By contrast, the existence of a non-trivial logarithmic correction is unexpected, in particular since such a correction does not appear in the exact result $\pi_{\max }^{\mathrm{HoC}}=(L+1)^{-1}$ for the HoC model $(\alpha=\mu=1)$. Remarkably, the logarithmic factors precisely cancel in this particular case.

\section{B Variational analysis at the maximum of $\lambda_{k}^{\mathrm{AN}}$}

In Fig. 4 we observed that $\lambda_{2}^{\mathrm{AN}}$ for the negative gamma distribution with shape parameter $s$ is maximized at $s=1 / 2$. Furthermore, we claimed that this can be naturally generalized to arbitrary values of $k$ if we replace the shape parameter by $1 / k$. As a next question, one might further ask if $\lambda_{k}^{\mathrm{AN}}$ is an extremum also with 
respect to arbitrary variations in the space of base fitness distributions $p_{f}$. Here, we prove that this is indeed the case for distributions with support limited to the negative real axis.

Let us first evaluate the $k$-fold convolution of the gamma distribution needed to compute Eq. (42). This is easily achieved using the property that the gamma distribution is closed under the convolution operation, i.e., the $k$-fold convolution of the gamma distribution with shape parameter $s$ is the gamma distribution with shape parameter $s k$. If we choose as our base distribution the negative gamma distribution with shape parameter $s=1 / k$,

$$
p_{f}(x)=p_{1 / k}(x) \equiv g_{1 / k}(-x),
$$

the $k$-fold convolution yields the gamma distribution with unit shape parameter a.k.a. a (negative) exponential distribution, characterized by the $\operatorname{CDF} \tilde{F}_{1 / k}^{(k)}(z)=e^{z}$ for $z<0$. Since $\tilde{F}_{1 / k}^{(k)}\left(y_{1}+y_{2}+\cdots\right)=e^{y_{1}} e^{y_{2}} \cdots$, Eq. 42 is fully factorized as

$$
\pi_{\max }^{\mathrm{AN}}=\left(\int d y g_{1 / k}(-y) e^{k y}\right)^{L}=(k+1)^{-L / k},
$$

which is exactly the result for the block model obtained in Eq. 26.

Next, let us derive a useful general formula for $\tilde{F}^{(k)}(z)$. Using the convolution theorem, it satisfies

$$
\tilde{F}^{(k)}(z)=\int_{-\infty}^{z} d z^{\prime} \int_{z^{\prime}}^{\infty} d y p_{f}(y) p_{f}^{(k-1)}\left(z^{\prime}-y\right)
$$

where $p_{f}^{(k-1)}(z)$ is the PDF of the $k-1$ fold convolution of $p_{f}(z)$. It will later be convenient to exchange the order of integrals:

$$
\begin{aligned}
\tilde{F}^{(k)}(z) & =\int_{-\infty}^{z} d y p_{f}(y) \int_{-\infty}^{y} d z^{\prime} p_{f}^{(k-1)}\left(z^{\prime}-y\right)+\int_{z}^{\infty} d y p_{f}(y) \int_{-\infty}^{z} d z^{\prime} p_{f}^{(k-1)}\left(z^{\prime}-y\right) \\
& =\int_{-\infty}^{z} d y p_{f}(y)+\int_{z}^{\infty} d y p_{f}(y) \tilde{F}_{s}^{(k-1)}(z-y) .
\end{aligned}
$$

In the first equality, we split the integral into two pieces to accommodate the condition $p_{f}^{(k-1)}(z)=0$ for positive $z$. In the next equality, we have used the fact that $\tilde{F}^{(k-1)}(0)=1$.

Now, we want to show that $\pi_{\max }^{\mathrm{AN}}$ is maximized when the base fitness distribution is given by Eq. (106). To this end, let us introduce a small perturbation $p_{f}(y)=$ $p_{1 / k}(y)+\epsilon \eta(y)$, with the properties that $\int d y \eta(y)=0$ and $\eta(y)=0$ for $y>0$. Since the probability Eq. 42 is given by the product of $2 L$ terms, there will be $2 L$ linear terms in $O(\epsilon)$, i.e. $\pi_{\max }^{\mathrm{AN}}$ changes by

$$
\begin{aligned}
\delta \pi_{\max }^{\mathrm{AN}}= & \epsilon L \int d y \eta(y) \int\left(\prod_{r=2}^{L} d y_{r} p_{1 / k}\left(y_{r}\right)\right) \prod_{l=0}^{L-1} \tilde{F}_{1 / k}^{(k)}\left(\sum_{m=1}^{k} y_{(l+m) \bmod L}\right) \\
& +L \int\left(\prod_{r=1}^{L} d y_{r} p_{1 / k}\left(y_{r}\right)\right) \delta \tilde{F}^{(k)}\left(\sum_{m=1}^{k} y_{m}\right) \prod_{l=1}^{L-1} \tilde{F}_{1 / k}^{(k)}\left(\sum_{m=1}^{k} y_{(l+m) \bmod L}\right) \\
\equiv & L\left(J_{1}+J_{2}\right) .
\end{aligned}
$$


The first term is straightforward to evaluate. Since $\tilde{F}_{1 / k}^{(k)}\left(\sum_{m=1}^{k} y_{(l+m) \bmod L}\right)$ is factorized, it readily follows that

$$
\begin{aligned}
J_{1} & =\epsilon \int d y \eta(y) \int\left(\prod_{r=1}^{L-1} d y_{r} p_{1 / k}\left(y_{r}\right)\right) \prod_{l=0}^{L-1} \tilde{F}_{1 / k}^{(k)}\left(\sum_{m=1}^{k} y_{(l+m) \bmod L}\right) \\
& =\epsilon \int d y \eta(y) e^{k y}(k+1)^{-(L-1) / k} .
\end{aligned}
$$

To evaluate $J_{2}$, let us rewrite it in the following way:

$$
\begin{aligned}
J_{2} & =\int\left(\prod_{r=1}^{L} d y_{r} p_{1 / k}\left(y_{r}\right)\right) \delta \tilde{F}^{(k)}\left(\sum_{m=1}^{k} y_{m}\right) \prod_{l=1}^{L-1} \tilde{F}_{1 / k}^{(k)}\left(\sum_{m=1}^{k} y_{(l+m) \bmod L}\right) \\
& =(k+1)^{-(L-k) / k} \int\left(\prod_{r=1}^{k} d y_{r} p_{1 / k}\left(y_{r}\right) e^{(k-1) y_{r}}\right) \delta \tilde{F}^{(k)}\left(\sum_{m=1}^{k} y_{m}\right)
\end{aligned}
$$

The argument of $\delta \tilde{F}^{(k)}$ is the sum of the variables $y_{r}$ that remain to be integrated over. To make them independent, let us introduce a delta function through the identity

$$
1=\int d Y \delta\left(\sum_{m=1}^{k} y_{m}-Y\right) \Theta(-Y)
$$

or, in the Fourier representation,

$$
1=\int \frac{d Y d Z}{2 \pi} e^{-i Z\left(\sum_{m=1}^{k} y_{m}-Y\right)} \Theta(-Y),
$$

where we impose the negativity of $Y$ by inserting an additional theta function. Using the property $\int d x \delta(x-a) f(x)=\int d x \delta(x-a) f(a)$, we may now complete the integrations over the $y_{r}$ as

$$
\begin{aligned}
& \int \frac{d Y d Z}{2 \pi} \Theta(-Y) \int\left(\prod_{r=1}^{k} d y_{r} g_{1 / k}\left(y_{r}\right) e^{(k-1) y_{r}}\right) e^{i Z\left(Y-\sum_{m}^{k} y_{m}\right)} \delta \tilde{F}^{(k)}(Y) \\
= & \int \frac{d Y d Z}{2 \pi} \Theta(-Y)(k-i Z)^{-1} e^{i Z Y} \delta \tilde{F}^{(k)}(Y)=\int d Y \Theta(-Y) e^{k Y} \delta \tilde{F}^{(k)}(Y),
\end{aligned}
$$

where we used Jordan's lemma to evaluate the integral with respect to $Z$. With this result, $J_{2}$ is of the relatively simple form

$$
J_{2}=(k+1)^{-(L-k) / k} \int d Y \Theta(-Y) e^{k Y} \delta \tilde{F}^{(k)}(Y) .
$$

Next, let us evaluate $\delta \tilde{F}^{(k)}(z)$. Using Eq. 109, we find that

$$
\begin{aligned}
\delta \tilde{F}^{(k)}(z) & =\epsilon k\left[\int_{-\infty}^{z} d y \eta(y)+\int_{z}^{\infty} d y \eta(y) \tilde{F}_{1 / k}^{(k-1)}(z-y)\right] \\
& =\epsilon k\left[\int_{-\infty}^{\infty} d y \eta(y)+\int_{z}^{\infty} d y \eta(y)\left(\tilde{F}_{1 / k}^{(k-1)}(z-y)-1\right)\right] \\
& =\epsilon k \int_{z}^{\infty} d y \eta(y)\left(\tilde{F}_{1 / k}^{(k-1)}(z-y)-1\right) \\
& =\epsilon k \int_{-\infty}^{\infty} d y \eta(y)\left(\tilde{F}_{1 / k}^{(k-1)}(z-y)-1\right) \Theta(y-z),
\end{aligned}
$$


where the factor $k$ comes from the $k$ different choices of $p_{f}(y)$ in the variation of $\tilde{F}^{(k)}$ and the fact that $\int d y \eta(y)=0$ is used to eliminate the first term in the second equality. As expected, this implies that any perturbation made in the range $(-\infty, z)$ does not change the behavior of $\tilde{F}^{(k)}(z)$. Inserting this result into $J_{2}$ gives

$$
\begin{aligned}
J_{2}= & (k+1)^{-(L-k) / k} \int d Y \Theta(-Y) e^{k Y} \int d y \eta(y) \\
& \times \epsilon k\left(\tilde{F}_{1 / k}^{(k-1)}(Y-y)-1\right) \Theta(y-Y) .
\end{aligned}
$$

Now, the only technical point left is the integration with respect to $Y$. The integral domain is determined by two theta functions $\Theta(-Y)$ and $\Theta(y-Y)$, but since $\eta(y)$ is assumed to be supported only on the negative real axis, the condition imposed by $\Theta(-Y)$ is irrelevant. Finally, using the identity

$$
\int_{-\infty}^{0} d Y k e^{k Y}\left(1-\frac{\Gamma\left(\frac{k-1}{k},-Y\right)}{\Gamma\left(\frac{k-1}{k}\right)}\right)=(k+1)^{\frac{1}{k}-1},
$$

we find

$$
J_{2}=-\epsilon \int d y \eta(y) e^{k y}(k+1)^{-(L-1) / k}
$$

Thus, the two terms in Eq. 110 perfectly cancel, which completes the proof that $\delta \pi_{\max }^{\mathrm{AN}}=0$.

\section{$\mathrm{C}$ General bounds on $\beta$ for uniform and regular structures with Gaussian fitness}

In this appendix we derive some general upper and lower bounds on the coefficient $\beta$, defined in Eq. (86), for NK structures that are both uniform and regular. For this purpose we write the probability of $\sigma$ being a local optimum as

$$
\pi_{\max }=\mathbb{E}\left[\prod_{l=1}^{L} \Theta\left(-\Delta_{l} F(\sigma)\right)\right]=\mathbb{E}\left[\prod_{l=1}^{L} \Theta\left(-\sum_{r=1}^{|\mathcal{B}|}\left(f_{r}\left(\downarrow_{B_{r}} \Delta_{l} \sigma\right)-f_{r}\left(\downarrow_{B_{r}} \sigma\right)\right)\right)\right] .
$$

All fitness values of the partial landscapes $f_{r}$ are i.i.d. random variables. If $l \in B_{r}$, then $f_{r}\left(\downarrow_{B_{r}} \Delta_{l} \sigma\right)$ and $f_{r}\left(\downarrow_{B_{r}} \sigma\right)$ are independent. Otherwise they are identical. Thus effectively only the sum over $r$ with $l \in B_{r}$ remains. Due to regularity there are $\tilde{k}=\frac{N k}{L}$ such elements for each $l$. For different $r$, the terms are always independent. The left-hand terms are also independent for different $l$. However the right-hand terms are correlated for different $l$ but the same $r$, resulting in a non-trivial problem. Using these observations we can directly integrate out all terms $f_{r}\left(\downarrow_{B_{r}} \Delta_{l} \sigma\right)$ and arrive at

$$
\pi_{\max }=\mathbb{E}\left[\prod_{l=1}^{L} \Phi_{\tilde{k}}\left(\sum_{r \mid l \in B_{r}} f_{r}\left(\downarrow_{B_{r}} \sigma\right)\right)\right],
$$

where $\Phi_{\tilde{k}}$ is the cumulative distribution function of the sum of $\tilde{k}$ i.i.d. fitness values. Introducing the short-hand notation $x_{r}=f_{r}\left(\downarrow_{B_{r}} \sigma\right)$, we can write the sum as a matrix product

$$
\pi_{\max }=\mathbb{E}\left[\prod_{l=1}^{L} \Phi_{\tilde{k}}\left((\mathbf{B} x)_{l}\right)\right]
$$


where $\mathbf{B}$ is the incidence matrix of the NK structure, i.e. $\mathbf{B}_{l r}=b_{l, r}=1$ if $l \in B_{r}$ and 0 otherwise.

If the base fitness distribution is a standard normal distribution, then the sum of $\tilde{k}$ i.i.d. fitness values is also normal distributed with variance $\tilde{k}$. Consequently we can simplify as

$$
\pi_{\max }=\mathbb{E}\left[\prod_{l=1}^{L} \Phi\left(\frac{1}{\sqrt{\tilde{k}}}(\mathbf{B} x)_{l}\right)\right] .
$$

The random vector $y=\frac{1}{\sqrt{\tilde{k}}} \mathbf{B} x$ is then jointly normal distributed with zero mean and covariance matrix $\mathbf{C}=\frac{1}{\tilde{k}} \mathbf{B B}^{T}$. This matrix is positive-semidefinite, and therefore

$$
\pi_{\max }=\int_{\mathbb{R}^{L}} \frac{\mathrm{d} y}{\sqrt{(2 \pi)^{L} \operatorname{det} \mathbf{C}}} \exp \left(-\frac{1}{2} y^{T} \mathbf{C}^{-1} y+\sum_{l=1}^{L} \ln \Phi\left(y_{l}\right)\right) .
$$

We can shift the integrand by a yet to be specified vector $z$, which yields

$$
\begin{array}{r}
\pi_{\max }=\int_{\mathbb{R}^{L}} \frac{\mathrm{d} y}{\sqrt{(2 \pi)^{L} \operatorname{det} \mathbf{C}}} \times \\
\times \exp \left(-\frac{1}{2} y^{T} \mathbf{C}^{-1} y-\frac{1}{2} z^{T} \mathbf{C}^{-1} z-z^{T} \mathbf{C}^{-1} y+\sum_{l=1}^{L} \ln \Phi\left(y_{l}+z_{l}\right)\right) .
\end{array}
$$

Absorbing the first term in the exponent into a probability measure, we have again

$$
\pi_{\max }=e^{-\frac{1}{2} z^{T} \mathbf{C}^{-1}} z_{\mathbb{E}}\left[\exp \left(-z^{T} \mathbf{C}^{-1} y+\sum_{l=1}^{L} \ln \Phi\left(y_{l}+z_{l}\right)\right)\right]
$$

where $y$ is still jointly normal distributed with covariance matrix $\mathbf{C}$.

Notice that the all-ones vector $\overline{1}$ is an eigenvector of $\mathbf{C}$ with the eigenvalue $k$. This can be seen through the relations $\mathbf{B} \overline{1}=\tilde{k} \overline{1}$ and $\mathbf{B}^{T} \overline{1}=k \overline{1}$, as there are exactly $\tilde{k}$ ones in each row of $\mathbf{B}$ and $k$ ones in each column. Thus let the $z_{l}=\bar{z}$ be equal for all $l$. Then

$$
\pi_{\max }=e^{-L \frac{\bar{z}^{2}}{2 k}} \prod_{l=1}^{L} \mathbb{E}\left[\exp \left(\sum_{l=1}^{L}\left(\ln \Phi\left(y_{l}+\bar{z}\right)-\frac{\bar{z}}{k} y_{l}\right)\right)\right] .
$$

\section{C.1 Lower bound}

By Jensen's inequality we have

$$
\pi_{\max } \geq e^{-L \frac{\bar{z}^{2}}{2 k}} \prod_{l=1}^{L} \exp \left(\mathbb{E}\left[\ln \Phi\left(y_{l}+\bar{z}\right)-\frac{\bar{z}}{k} y_{l}\right]\right) .
$$

Because $y_{l}$ has a symmetric distribution, the mean of $\bar{z} y_{l}$ vanishes. The variance of $y_{l}$ is always 1 , because by regularity and uniformity the diagonal elements of $\mathbf{B B}^{T}$ are $\tilde{k}$, which is canceled to 1 by the pre-factor in $\mathbf{C}$. If we then assume $\bar{z}$ to be increasing in our limit of interest and noting that the Gaussian has a tail falling much quicker to zero than the tail of $\ln \Phi$ falls to $-\infty$ at $x \rightarrow-\infty$, we can establish the bound

$$
\pi_{\max } \geq e^{-L \frac{\bar{z}^{2}}{2 k}} \prod_{l=1}^{L} \exp \left(\mathbb{E}\left[\Phi\left(y_{l}+\bar{z}\right)-1\right](1+o(1))\right)
$$


which can be evaluated to

$$
\pi_{\max } \geq \exp \left(-L \frac{\bar{z}^{2}}{2 k}+L\left(\Phi\left(\frac{\bar{z}}{\sqrt{2}}\right)-1\right)(1+o(1))\right) .
$$

If we choose $\bar{z}=2 \sqrt{\ln k}$, then asymptotically for large $k$

$$
\pi_{\max } \geq \exp \left(-L\left(\frac{2 \ln k}{k}+\mathcal{O}\left(\frac{1}{k \sqrt{\ln k}}\right)\right)\right) .
$$

Note that choosing $\bar{z}=\tilde{z} \sqrt{\ln k}$ with $\tilde{z}<2$ will not give a better bound, as the right-hand term in the exponent in Eq. 131 would then dominate and approach zero more slowly than $\frac{\ln k}{k}$. This shows that $\beta \leq 2$ for uniform and regular structures. With the MF model, which is uniform and regular, we have an example of a realization of $\beta=2$. This shows that the bound is tight.

\section{C.2 Upper bound}

Starting from Eq. (128) we can find an upper bound by simply optimizing each term in the sum. The resulting sum is then an upper bound on the integrand, and because the expectation is taken with respect to a probability measure, it is bounded by the same value as well. If $0<\frac{\bar{z}}{k}<\frac{1}{\sqrt{2 \pi}}$, the optimum must be at $y_{l}^{\star}+\bar{z}>0$. Then by using the simplification $\ln \Phi\left(y_{l}+z_{l}\right) \leq \Phi\left(y_{l}+z_{l}\right)-1$, the optimum is found to be at

$$
y_{l}^{\star}=\sqrt{2 \ln \left(\frac{k}{\sqrt{2 \pi} \bar{z}}\right)}-\bar{z} .
$$

Inserting $y_{l}^{\star}$ back into the simplified argument of the expectation and assuming $\bar{z} \rightarrow \infty$ in the limit of interest we find

$\pi_{\max } \leq \exp \left(-L \frac{\bar{z}^{2}}{2 k}-L\left(\frac{\bar{z}}{k \sqrt{2 \ln \left(\frac{k}{\sqrt{2 \pi} \bar{z}}\right)}}(1+o(1))+\frac{\bar{z}}{k} \sqrt{2 \ln \left(\frac{k}{\sqrt{2 \pi} \bar{z}}\right)}-\frac{\bar{z}^{2}}{k}\right)\right)$.

The left-most and right-most terms are of equal order, but the second one from the left is always of less significant order than the second from the right, as long as $\bar{z}=o(k)$.

The second term from the right becomes equal in order to the other two if $\bar{z}=\tilde{z} \sqrt{2 \ln k}$ with a positive constant $\tilde{z}$. This satisfies the condition $\bar{z}=o(k)$ while still $\bar{z} \rightarrow \infty$, as required by previous assumptions (given that $k \rightarrow \infty$ in the limit of interest). With this we have

$$
\pi_{\max } \leq \exp \left(-L\left(\frac{\ln k}{k}\left(2 \tilde{z}-\tilde{z}^{2}\right)+\mathcal{O}\left(\frac{\ln \ln k}{k}\right)\right)\right) .
$$

The bound is best for $\tilde{z}=1$, and so:

$$
\pi_{\max } \leq \exp \left(-L\left(\frac{\ln k}{k}+\mathcal{O}\left(\frac{\ln \ln k}{k}\right)\right)\right)
$$

showing that $\beta \geq 1$ for regular and uniform NK structures with Gaussian fitness. This bound is realized by the AN and BN structures, for example, and thus it is tight. 


\section{Simulation of the number of local maxima}

As first realized in [6, the choice of a Gaussian base fitness distribution greatly simplifies the computation of $\pi_{\max }$ through the numerical evaluation of Eq. (25), as it allows us to take advantage of an efficient algorithm. With this choice, the integrals over $\mathbf{q}$ and $\mathbf{y}$ can be cast into the form of multi-dimensional Gaussian integrals which may be evaluated for generally defined NK structures. Once these integrals are evaluated, we may construct a covariance matrix $\Sigma$ that satisfies the relation

$$
\pi_{\max }=\int \mathcal{D} \mathbf{u} \exp \left(-\frac{1}{2} \sum_{j l} u_{j} \Sigma_{j l}^{-1} u_{l}\right),
$$

where $\int \mathcal{D} \mathbf{u}=\frac{1}{\sqrt{(2 \pi)^{L} \operatorname{det} \Sigma}} \int_{0}^{\infty} \prod_{j} d u_{j}$ and the matrix elements of $\Sigma$ are given by

$$
\Sigma_{j l}= \begin{cases}2 \sum_{r} b_{l, r} & j=l \\ \sum_{r} b_{j, r} b_{l, r} & j \neq l\end{cases}
$$

Thus, the problem reduces to determining the probability that all the entries of the Gaussian random vector realized by the covariance matrix $\Sigma$ are positive. Since finding the probability for rectangular domains of multivariate Gaussian distribution is a well-known problem, an efficient algorithm has been known for a long time 22 and its implementation has been provided by the original authors as an R library 23 .

Roughly speaking, this algorithm consists of two steps: i) transforming to an integral over a unit rectangular domain such that a rejection-free Monte-Carlo simulation is possible and ii) finding an ordering of loci that minimizes the variance of the Monte-Carlo step. However, since the loci in the NK models we consider in this review are statistically identical, the second step is irrelevant in this particular case. Thus, here we describe briefly how the transformation can be achieved from Eq. 137).

Since $\Sigma$ is positive-definite, the Cholesky decomposition ensures that there exists a triangular matrix $C$ such that $\Sigma=C C^{T}$. The substitution $\mathbf{u}=C \mathbf{x}$ then diagonalizes the integral at the cost of nontrivial integral domain,

$$
\pi_{\max }=\frac{1}{(2 \pi)^{L / 2}} \int_{\mathbf{x} \in \mathcal{R}} \prod_{j=1}^{L} d x_{j} \exp \left(-\frac{1}{2} \sum_{j=1}^{L} x_{j}^{2}\right),
$$

where the domain $\mathcal{R}=\left(a_{1}, \infty\right) \times\left(a_{2}, \infty\right) \times \cdots\left(a_{L}, \infty\right)$ and $a_{j}=-\sum_{l=1}^{j-1} x_{l} C_{j l} / C_{j j}$. Next, performing the canonical transformation to a standard uniform distribution $z_{i}=\Phi\left(x_{i}\right)$, where $\Phi(x)$ is the CDF of the standard Gaussian distribution, the integral becomes

$$
\pi_{\max }=\int_{\mathbf{z} \in \mathcal{R}^{\prime}} \prod_{j=1}^{L} d z_{j}
$$

where $\mathcal{R}^{\prime}=\left(d_{1}, 1\right) \times\left(d_{2}, 1\right) \times \cdots\left(d_{L}, 1\right)$ and $d_{j}=\Phi\left(-\sum_{l=1}^{j-1} \Phi^{-1}\left(z_{l}\right) C_{j l} / C_{j j}\right)$. Finally, another linear transformation $z_{j}=d_{j}+w_{j}\left(1-d_{j}\right)$ brings the integral into the form

$$
\pi_{\max }=\int_{\mathbf{w} \in \mathcal{R}^{\prime \prime}} \prod_{j=1}^{L}\left(1-d_{j}\right) d w_{j},
$$

where $\mathcal{R}^{\prime \prime}=(0,1)^{L}$. Now that the integral domain is the $L$-dimensional unit rectangle, this integral can be evaluated by sampling $L$ random variables from a uniform distribution on $(0,1)$ and subsequently estimating the weight factors $d_{j}$. 